\title{
Dynamin2: A novel regulator of lamellipodial and lamellar actin networks in non-muscle cells
}

\author{
Manisha Menon \\ Kochi, India \\ B.Sc. Medical Biotechnology, Manipal University, India, 2005 \\ M.Sc. Medical Biotechnology, Manipal University, India, 2007 \\ A Dissertation presented to the Graduate Faculty \\ of the University of Virginia in Candidacy for the Degree of \\ Doctor of Philosophy \\ Department of Biology
}

August 2013 


\begin{abstract}
How the actin cytoskeleton is organized in a cell is critical for regulation of cell migration, maintenance of cell shape, and endocytosis. In a migrating cell, actin exists in two organizational domains: a highly dynamic and branched network in the lamellipodia, extending 1-3 $\mu \mathrm{m}$ in from the cell edge; and a stable, bundled actin network in the lamella that exists further back from the lamellipodia, towards the nucleus. Bundled actin in the lamella associates with non-muscle myosin II to form an actomyosin network, which consists of three types of structures: transverse arcs, dorsal stress fibers and ventral stress fibers. Proper organization of the actomyosin network regulates rearward flow of transverse arcs towards the nucleus - termed actomyosin retrograde flow. Actomyosin network organization can also facilitate engagement with and maturation of focal adhesions, multi-protein complexes linking actin network and the underlying substratum.

An overarching goal has been to elucidate the relationship between actin networks in the lamellipodia and lamella and to clearly understand how the lamellar actomyosin networks are generated. One idea postulates that actin in the lamellipodia disassembles completely and lamellar networks are then synthesized de novo. Other evidence however argues that a component of the lamellipodial actin contributes to generation of the lamella. An outstanding goal of this research is to understand the mechanisms by which lamellipodial actin filaments are efficiently remodeled from their branched organization within the lamellipodia to one of parallel filament bundles in the lamella and to elucidate the proteins involved in this process.
\end{abstract}

Previous work in our lab and by others had uncovered an unconventional actinregulatory protein, dynamin2. In vitro studies demonstrated a role for dynamin2 in 
bundling and reorganization of, or remodeling, of F-actin and studies in fixed cells implicated dynamin2 in actomyosin organization. However, to elucidate how dynamin2 regulates the dynamic process of actomyosin assembly, I used live-cell imaging and siRNA-mediated depletion of endogenous dynamin2 to investigate a function for dynamin2 in remodeling lamellipodial and lamellar actin networks in vivo. Nascent myosin puncta appeared at an increased rate during actomyosin assembly in dynamin2depleted U2-OS cells and organized poorly at the lamellipodium-lamellum boundary. Also, the rate of myosin retrograde flow was high compared with that of control siRNAtreated U2-OS cells. These indicated a poorly organized actomyosin network. However, dynamin2 did not localize to the actomyosin network. Instead, GFP-dynamin2 localized to the distal lamellipodial edge in live cells and regulated the spatiotemporal distribution of the actin cross-linker $\alpha$-actinin at the lamellipodia. Therefore, I postulated a function for dynamin2 in reorganizing lamellipodial F-actin to set up an actin architecture that creates an ideal template for actomyosin formation within the lamellum.

In order to elucidate a mechanism for how dynamin2 influenced lamellipodial Factin, various fluorescently-tagged dynamin2 mutants were assessed for rescue of the defects in lamellar actomyosin and lamellipodial $\alpha$-actinin. Direct interaction of dynamin2 with $\mathrm{F}$-actin and $\mathrm{C}$-terminal proline rich domain (PRD) and the rate of dynamin2-dependent GTP hydrolysis were critical for regulating the spatiotemporal distribution of lamellipodial $\alpha$-actinin and for rescuing high actomyosin retrograde flow. Importantly, to rule out secondary effects on actin networks via defects in endocytosis, I demonstrated that dynamin 2 depletion did not affect internalization of integrin $\beta 1$ and transferrin receptor. This work establishes dynamin2 as a critical regulator of global actin 
networks. This novel function for dynamin2 can have far-reaching implications in regulating the actin cytoskeleton for important functions like cytokinesis, maintaining cellular junctions and membrane modulations during endocytosis 


\section{Dedication}

There are a lot of people I would like to dedicate this thesis to. First, to my husband Ankit Malhotra for his patience and for always being by my side. Thank you for being the eternal optimist.

I also dedicate this thesis to my parents, who have always supported the choices I made, including the decision to re-locate halfway across the world to pursue my $\mathrm{PhD}$. A big hug and thank you to my brother Umesh for making me laugh and making me think. I also thank my parents-in-law and my brother-in-law Anuj Malhotra, for simply being amazing.

I would also like to thank my grandparents, who have always been proud of my achievements however small, with a special dedication to my grandfather Mr. P. N. Menon who passed away three years back. I will always miss him and want to thank him for his unconditional love and support.

I dedicate this thesis to my $\mathrm{PhD}$ advisor Dr. Dorothy Schafer, who has demonstrated by example how to formulate and pursue the most important scientific question and arrive at a reasonable conclusion. Also, thank you for teaching me the importance of keeping an open mind. Thank you to all members of Schafer lab, both past and present.

Finally, I dedicate this thesis to my friends, who have made my six years in Charlottesville a wonderful and truly memorable experience. Thank you for the laughs and the conversations, both silly and serious. 


\section{Table of Contents}

\section{Page number}

$\begin{array}{lr}\text { Abstract } & 1 \\ \text { Dedication } & 4 \\ \text { Table of Contents } & 5 \\ \text { List of Figures } & 7 \\ \text { Movie Legends } & 10 \\ \text { Chapter 1 - Introduction } & 12 \\ \text { Chapter 2 - } & 40\end{array}$

Dynamin: expanding its scope to the cytoskeleton

Chapter 3 -

Dynamin2 remodels lamellipodial actin networks to

orchestrate lamellar actomyosin

Introduction

95

Results

98

Discussion

Materials and Methods

References

Chapter 4 -

Discussion and Future Perspectives

References

Dynamin2 does not influence

levels of active, GTP-bound small GTPases 
Regulation of cell migration by dynamin 2 


\section{List of Figures}

\section{Page number}

\section{Chapter 1}

Figure 1: Electron microscopy (EM) image depicting actin organization in lamellipodial and lamellar actin networks

Figure. 2: The semi-sarcomeric pattern of myosin light

chain 2 (MLC-2) and $\alpha$-actinin observed in transverse arcs of a U2-OS cell.

Figure. 3: Dynamin2 localizes to the distal edge of the lamellipodia in live U2OS cells.

\section{Chapter 2}

Figure. 1: The various ways by which dynamin influences cytoskeletal networks.

\section{Chapter 3}

Figure 1: Dynamin2 influences actomyosin assembly in U2-OS cells.

Figure 2: Depleting dynamin2 does not significantly affect the rate of internalization of integrin $\beta 1$ or transferrin receptor.

Figure 3: Targeting dynamin2 to the distal lamellipod depends on direct interactions with F-actin and the 
C-terminal PRD.

Figure 4: Dynamin1 is detected at diffraction-limited cytoplasmic punctae but not at the lamellipodia.

Figure 5: Dynamin2 influences the spatiotemporal organization of $\alpha$-actinin in lamellipodia.

Figure 6: The spatial distribution of cortactin within lamellipodia and in the cytoplasm is altered in dyn2-depleted cells.

Figure 7: Dynamin2 influences coupling of transverse arcs and adhesions.

Figure 8: Dynamin2-dependent organization of GFP- $\alpha$-actinin within lamellipodia depends on F-actin binding, the PRD and finely tuned GTP hydrolysis.

Figure 9: Dynamin2-dependent regulation of lamellar actomyosin retrograde flowdepends on F-actin binding, the PRD and its rate of GTP hydrolysis.

Figure 10: GTP hydrolysis by dynamin 2 influences protrusion dynamics.

Figure. 11: GFP-FAK does not localize to the distal lamellipod

\section{Chapter 4}

Figure 1: Working model for dynamin2-dependent reorganization of actin networks at the lamellipodia

\section{Appendix I}


Figure 1: Dynamin2 depletion does not alter levels of active GTPases.

\section{Appendix II}

Figure 1: Dynamin2 regulates persistence of migration

during random cell motility.

Figure. 2: Dynamin2 influences directed cell migration

into a wound

Figure. 3: Dynamin2 regulates lamellipodial protrusive

behavior 


\section{Movie Legends}

Movie 1. Representative control siRNA-treated (left) and dyn2-siRNA-treated (right) U2-OS cells transiently expressing GFP-myosin light chain 2 (MLC2) (green) and mCh- $\alpha$-actinin (red). Images were collected at a single focal plane every $10 \mathrm{~s}$ using an EM-CCD camera $(512 \times 512$ pixels) and presented at 200X real-time.

Movie 2. Representative dynamin2-depleted U2-OS cell transiently expressing GFP-WT-dyn2 (green) and $\mathrm{mCh}$ - $\alpha$-actinin (red). Images were collected at a single focal plan every $3 \mathrm{~s}$ using an ORCA ER CCD camera and presented at 60X real-time.

Movie 3. Representative U2-OS cell transiently expressing GFP-WT-dyn2 (green) and mCh- $\alpha$ actinin (red). This movie shows the transient association of GFP-dyn2 with ruffles and filopodia. Images were collected at a single focal plane every $3 \mathrm{~s}$ using an ORCA ER CCD camera and presented at $60 \mathrm{X}$ real-time.

Movie 4. Representative dynamin2-depleted U2-OS cell transiently expressing mCh-dyn2- $\mathrm{K}_{5} \mathrm{E}_{5}$ (green) and GFP- $\alpha$-actinin (red). Note that the individual channels were pseudo-colored to make them consistent with other panels of Fig. 3 in the text. Images were collected at a single focal plane every $5 \mathrm{~s}$ using an ORCA ER CCD camera and presented at 60X real-time.

Movie 5. Representative dynamin2-depleted U2-OS cell transiently expressing GFP-dyn2- $\Delta$ PRD (green) and $\mathrm{mCh}-\alpha$-actinin (red). Images were collected at a single focal plane every 3 seconds using an ORCA ER CCD camera and presented at $60 \mathrm{X}$ real-time. 
Movie 6. Representative control- and dyn2-siRNAi-treated U2-OS cells transiently expressing GFP- $\alpha$-actinin. Images were collected at a single focal plane every $5 \mathrm{~s}$ using an ORCA ER CCD camera and presented at 100X real-time.

Movie 7. Representative control (left) and dynamin2-depleted (right) U2-OS cell transiently expressing GFP-paxillin (green) and $\mathrm{mCh}-\alpha$-actinin (red). Images were collected at a single focal plane every $10 \mathrm{~s}$ using an EM-CCD camera (512x512 pixel) and presented at 200X real-time.

Movie 8. Representative movies of GFP-MLC2 in control, dyn2-depleted and dyn2-depleted cells rescued with mutant dynamin 2 proteins as indicated. Mutant dynamin 2 proteins were tagged with mCherry (not shown). For each movie, an image stack (5-7 images, $0.5 \mu \mathrm{m}$ spacing) was collected every $10 \mathrm{~s}$ using an EM-CCD camera (512x512 pixel) and presented at 200X real-time.

Movie 9. Representative dynamin2-depleted U2-OS cells transiently expressing either GFPdyn2-T141A (green, left) or GFP-dyn2-S61D (green, right) and mCh- $\alpha$-actinin (red). Images were collected at a single focal plane every $3 \mathrm{~s}$ using an ORCA ER CCD camera and presented at 60X real-time. 
Chapter 1

Introduction 


\section{Introduction}

In a cultured cell undergoing migration, there are two opposing forces in play the protrusive force due to actin polymerization that impinges on the cell membrane and the backward-pulling force exerted by the tension on the membrane (Giannone et al., 2009; Schwarz and Gardel, 2012; Vicente-Manzanares et al., 2009a). Along with this membrane-exerted tension, there is rearward pulling by contractile lamellar actin networks. At the lamellipodia, polymerization of a branched, dendritic actin network creates the propulsive force for membrane protrusion. Proximal to the lamellipodia, lamellar actin networks contract and cause a rearward - or retrograde flow. The rate of retrograde flow is regulated by interactions with the substrate via focal adhesions, the physical link between the substrate and the overlying actin network. Efficient formation of the lamellar network involves association between actin filaments and the protein nonmuscle myosin II (Hotulainen and Lappalainen, 2006) and this network is also called the actomyosin. The lamellar actomyosin network consists of three kinds of structures: transverse arcs, dorsal stress fibers and ventral stress fibers, and these three types of structures together comprise the stress fiber architecture. They are important for maintenance of cell shape and exerting contractility-dependent tension for cell migration.

The lamellipodial and lamellar actin structures have been well studied, however there are still unanswered questions regarding the relationship between these two actin domains.. An outstanding question is what are the global changes in actin organization that occur to mediate a transition in architecture from highly branched and dendritic in the lamellipodia to parallel and bundled in the lamella. One mechanism posits that these domains are completely independent from one another, with the actin completely turned 
over at the base of the lamellipodia and lamellar actin synthesized de novo (Ponti et al., 2004; Vallotton and Small, 2009). Other work, however, postulates that part of the actin networks at the lamellipodia contribute to initiating formation of the lamellar actin (Anderson et al., 2008; Burnette et al., 2011; Hotulainen and Lappalainen, 2006; Nemethova et al., 2008; Shutova et al., 2012). In this research, I investigate a novel actin remodeler, dynamin2, and address its role in the reorganization of actin networks at the lamellipodia that in turn modulates actomyosin assembly.

Dynamin2 is a large GTPase involved in endocytosis and a classical view of its function involves oligomerization along necks of invaginating vesicles followed by GTP hydrolysis-dependent conformational change causing vesicle scission (Ferguson and De Camilli, 2012; Praefcke and McMahon, 2004). An emerging view of dynamin's function as a scission molecule involves formation of short dynamin oligomers in the presence of GTP, followed by multiple rounds of assembly and disassembly on lipid nanotubes that cause local reorganization of lipids eventually leading to scission (Bashkirov et al., 2008). A GTP hydrolysis-dependent powerstroke of the dynamin oligomer has also been implicated in causing a conformational change to the membrane scaffold, a precursor to membrane scission (Chappie et al., 2011).

There are three mammalian isoforms of dynamin. Dynamin1 localizes to neuronal tissues (Sontag et al., 1994), dynamin2 is the ubiquitous isoform (Cook et al., 1994) and dynamin3 is present in lungs, testes and neurons (Cook et al., 1996; Nakata et al., 1993). The isoforms are predominantly similar in sequence at their N-terminal GTPase domain, with differences in the membrane-binding pleckstrin-homology $(\mathrm{PH})$ domain and the Cterminal proline-rich domain (PRD) (Urrutia et al., 1997). A detailed analysis of 
dynamin's structure and biochemistry is covered in chapter 2. The dynamin isoforms have different splice variants (Cao et al., 1998; Urrutia et al., 1997). Isoform- and splice variant-specific functions for dynamin have been demonstrated in endocytosis and vesicular trafficking (Liu et al., 2008). In dynamin2-knockout mouse embryonic stem cells, re-expression of dynamin 1 did not rescue defects in clathrin-mediated endocytosis as efficiently as dynamin2, suggesting isoform specificity in the critical function of clathrin-mediated endocytosis in non-neuronal cells. On the other hand, the function of macropinocytosis could be rescued by both dynamin1 and dynamin2, suggesting a mechanism for dynamin function in fluid-based uptake that is different from endocytosis. Isoform specificity in endocytosis could arise due to specific lipid templates recognized by the isoforms. For example, dynamin1 was shown to be a membrane curvature generator in vitro whereas dynamin2 was more of a curvature sensor. This difference was attributed to a single amino acid difference between the two isoforms in the $\mathrm{PH}$ domain (Liu et al., 2011). Dynamin1 could be involved in rapid synaptic transmission in neuronal cells whereas dynamin2 could be important for the slower, constitutive function of clathrin-mediated endocytosis in non-neuronal cells (Liu et al., 2011). Because the dynamin isoforms exhibit maximum variability between their sequences in the C-terminal PRD (Urrutia et al., 1997), isoform-specific functions could be modulated by different PRD-binding partners.

An emerging view of dynamin, based on work from the past decade, is as a regulator of actin networks (Kruchten and McNiven, 2006; Menon and Schafer, 2013). There is evidence of dynamin modulating F-actin at clathrin-coated endocytic pits (Ferguson et al., 2009) and also at non-endocytic structures like actin comet tails (Lee 
and De Camilli, 2002; Orth et al., 2002), apical actomyosin contractile rings in epithelial cells (Chua et al., 2009) and as a regulator of actin polymerization in podocytes (Gu et al., 2010). The less-extensively studied isoform, dynamin3, is involved in synaptogenesis in cultured hippocampal neurons, with expression of the isoform dyn3baa forming actinrich, immature filopodial protrusions along the sides of maturing dendrites. Formation of the filopodial protrusions depends on interaction of dynamin3baa with the actin-binding protein cortactin, via dynamin3baa's PRD, suggesting a function for dynamin3baa in modulation of actin networks in cultured hippocampal neurons (Gray et al., 2005). In this work, I focus on dynamin2 influencing assembly and organization of actomyosin, as defects in actomyosin organization and flow are one of the prominent phenotypes observed following dynamin2 depletion in my cell system.

In the following sections, I will briefly discuss lamellipodial and lamellar actin architectures, what is known so far about the inter-relationship between lamellipodial and lamellar actin domains, the proteins present in these domains relevant to this work and finally present the rationale for this study.

\section{The lamellipodia}

The lamellipodia are highly dynamic, sheet-like regions that extend 1-3 $\mu \mathrm{m}$ in from the leading edge of migrating cells and contain characteristic branched actin networks (Pollard and Borisy, 2003; Svitkina, 2007) (Fig. 1). Rapid actin polymerization occurs close to the cell edge and this dynamic process powers protrusion of the leading edge of the cell during migration. Actin polymerization occurs with the faster growing, or barbed, ends of the filaments oriented towards the cell edge. The dendritic organization 
of filaments in the lamellipodia is facilitated by nucleation of new actin filaments from the sides of pre-existing 'mother' filaments at an angle of $70^{\circ} \mathrm{C}$ (Blanchoin et al., 2000). The multi-subunit actin nucleator Arp2/3 complex can nucleate actin polymerization from sides of actin filaments (Mullins et al., 1998). Electron microscopy studies in combination with gold-conjugated antibody staining have shown Arp2/3 localize to actin branch points in the lamellipodia (Svitkina and Borisy, 1999).

During cell migration, newly polymerized actin close to the membrane is ATPbound and following nucleotide hydrolysis, actin at the rear of the lamellipodia becomes ADP-bound. This ADP-bound actin at the lamellipodial rear is targeted by a group of actin depolymerizing proteins called ADF/cofilin (Bernstein and Bamburg, 2010; Svitkina and Borisy, 1999; Van Troys et al., 2008), which regenerates monomeric actin for maintaining actin polymerization at the leading edge (Pollard and Borisy, 2003). Immunofluorescence and gold-conjugated secondary antibody staining demonstrate cofilin localization at the rear of the lamellipodia (Svitkina and Borisy, 1999). Apart from $\mathrm{ADF} /$ cofilin, others proteins could also be involved in depolymerization of actin filaments. For example, two proteins coronin and Aip1 can facilitate cofilin binding to Factin and lower the cofilin concentration required for disassembly of actin comet tails in Listeria monocytogenes (Brieher et al., 2006).

\section{Lamellar actin networks}

Further away from the cell edge of migrating cells and closer to the nucleus, the actin network gets remodeled into a different conformation - an anti- parallel network of actin filaments (Fig. 1) where F-actin bundled by the actin cross-linking protein $\alpha$-actinin 
starts associating with myosin II to form structures called transverse arcs, part of the contractile unit of the lamellar cytoskeletal network (Tojkander et al., 2012). Apart from transverse arcs, the other types of lamellar actin structures are dorsal stress fibers and ventral stress fibers and these three together constitute the lamellar stress fiber machinery, or the actomyosin (Tojkander et al., 2012). It is important to note that not all cell types distinctly exhibit the different types of stress fibers. The cells used in this dissertation, U2-OS, have well-organized lamellar structures (Mooren et al., 2009; Oakes et al., 2012) and therefore are a good cell model to study lamellar actin formation and organization.

Dorsal stress fibers connect transverse arcs to focal adhesions. Dorsal stress fibers are generated via formin mDia-dependent actin polymerization at focal adhesions (Hotulainen and Lappalainen, 2006). In contrast, ventral stress fibers are connected to focal adhesions on both ends and can be generated from previously existing dorsal stress fibers or from transverse arcs (Hotulainen and Lappalainen, 2006).

A well-organized lamellar actin network can influence remodeling of the extracellular matrix (ECM), potentially via regulation of focal adhesion maturation (Oakes et al., 2012). Association between focal adhesions and the overlying actin stress fiber network modulates traction force exerted on the substratum (Giannone et al., 2009; Schwarz and Gardel, 2012). If the actin network is poorly organized, adhesion maturation is inefficient (Oakes et al., 2012) and the resultant weakly coupled actin stress fiber-focal adhesion linkage may not apply enough traction force on the substratum. This weak interaction could result in high actomyosin retrograde flow (Giannone et al., 2009; Schwarz and Gardel, 2012; Vicente-Manzanares et al., 2009a). 
Figure 1. Electron microscopy (EM) image depicting actin organization in lamellipodial and lamellar actin networks.

EM of a rotary shadowed cell following live-cell extraction. The two regions, area 1 and area 2, depict the different actin network organizations in the lamellipodia and lamella respectively. Scale bar: $1 \mu \mathrm{m}$. (Burnette et al., 2011) 
Figure. 1: Electron microscopy (EM) image depicting actin organization in lamellipodial and lamellar actin networks.

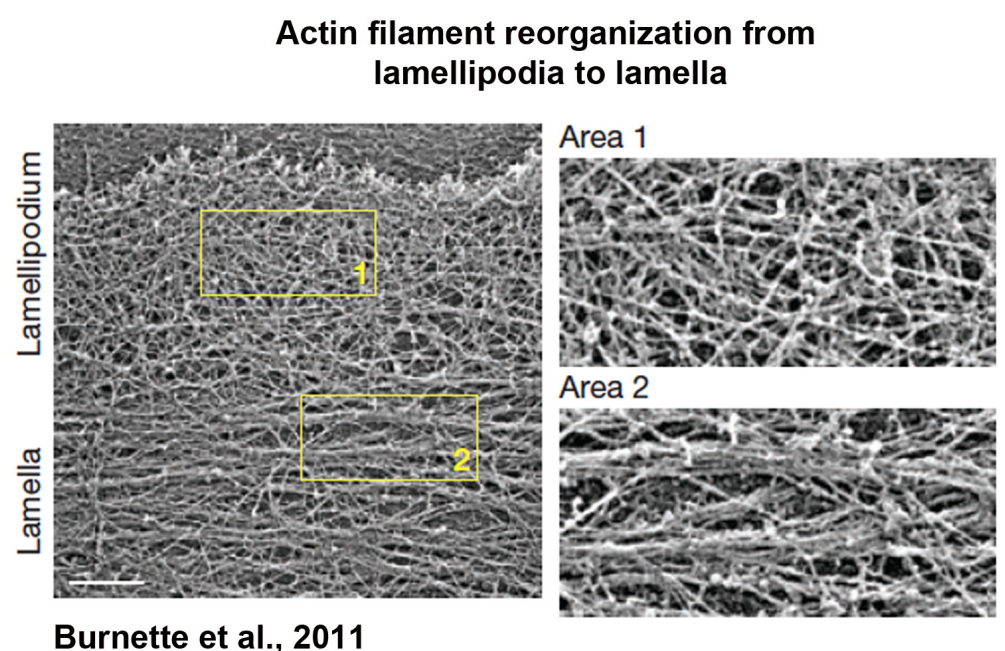




\section{$\underline{\text { Relationship between lamellipodial and lamellar actin networks }}$}

One of the overarching goals of this dissertation is to address how global reorganization of actin networks is regulated. One critical question to be addressed is the relationship between lamellipodial and lamellar actin networks. Speckle microscopy analysis in Ptk1 cells and newt lung epithelial cells demonstrated that in a migrating cell, actin exists in two kinetically and functionally distinct but spatially overlapping domains - the lamellipodia and lamella (Ponti et al., 2004). This work described lamellipodia as a thin sheet existing above the lamellar region, with the lamella extending all the way to the cell edge. The spatial overlap between lamellipodia and lamella was deduced from the observation that a proportion of longer-lived actin speckles, characteristic of less dynamic actin at lamella, co-existed with short-lived lamellipodial speckles at the leading edge of the cell (Ponti et al., 2004). This view was challenged five years later following reanalysis of the same image data, which did not demonstrate the presence of the longerlived actin speckles in the lamellipodia (Vallotton and Small, 2009). Instead, the reanalysis showed that lamellipodia and lamella exist as two spatially segregated populations in a migrating cell. Hence, there is a debate on the spatial relationship between lamellipodia and lamella, however the traditional view is of the lamella existing behind the lamellipodia, separated spatially and with distinct kinetics.

Another body of evidence suggests that instead of being completely turned over or disassembled, a part of lamellipodial actin networks - both dendritic networks in lamellipodia and needle-like actin bundles in the filopodia - contribute to formation of lamellar actomyosin networks (Anderson et al., 2008; Hotulainen and Lappalainen, 2006; 
Nemethova et al., 2008; Shutova et al., 2012). EM imaging and fluorescence microscopy have shed light on the possible ways by which networks in the lamellipodia give rise to lamellar actin networks.

According to one study, F-actin bundles cross-linked with $\alpha$-actinin appear at the proximal lamellipodia in the osteosarcoma cell line U2-OS and they start associating endto-end with myosin II at the lamellipodia-lamella interface, marking the beginning of actomyosin transverse arc formation in U2-OS cells (Hotulainen and Lappalainen, 2006). Timelapse imaging showed the gradual coalescence of $\alpha$-actinin and myosin puncta over time to form the semi-sarcomeric pattern characteristic of the actomyosin. This work also suggested that transverse arc formation was Arp2/3 dependent, whereas dorsal stress fibers elongated from focal adhesions and was formin mDia-mediated (Hotulainen and Lappalainen, 2006). Timelapse movies captured in my research have also observed the alternating semi-sarcomeric pattern of $\alpha$-actinin-bundled actin and myosin II in U2-OS cells (Fig. 2). Evidence of fluorescently labeled $\alpha$-actinin-cables exhibiting gaps that correspond to myosin II fluorescence in the lamella was shown almost 20 years back (Verkhovsky et al., 1995).

Another piece of elegant work using photo convertible actin-tdEos showed that in Ptk1 cells, a component of actin flowing rearward in the lamellipodia gradually formed nascent actin arcs, oriented parallel to the cell edge (Burnette et al., 2011). The authors observed myosin II appearing along retracting regions of lamellipodial protrusions and postulated a function for myosin II in locally reorganizing the retrogradely flowing lamellipodial actin to initiate nascent arc formation. These arcs associated with focal adhesions at the lamellipodia-lamella interface, which slowed down rate of flow of the 
arcs and also enabled protrusion of the cell front (Burnette et al., 2011). In my research, I propose a novel actin remodeling protein, dynamin2, which could locally remodel lamellipodial actin filaments present at the advancing edges of protrusions into a favorable conformation for downstream actin bundling and initiation of actomyosin formation.

Filopodia, dynamic spike-like projections consisting of bundled actin filaments, observed at the cell edge of certain cell types, also contribute to seeding the formation of lamellar actin structures (Anderson et al., 2008; Nemethova et al., 2008; Shutova et al., 2012). Filopodial bundles in the lamellipodia could act as precursors for formation of lamellar transverse arcs, as shown in REF52 cells re-forming their actomyosin following blebbistatin washout (Shutova et al., 2012). In migratory goldfish fibroblasts, filopodia that collapse into the lamellipodium initiate the formation of both actomyosin bundles and nascent adhesions (Nemethova et al., 2008). This process is dependent on myosin IIdependent contractility. In another system, migrating primary chick embryo fibroblasts, structures called graded polarity (GP) bundles form in the lamella, with their barbed ends oriented in the direction of migration (Anderson et al., 2008). These specialized lamellar actin structures also form from filopodial structures in the lamellipodia. 
Figure. 2: The semi-sarcomeric pattern of myosin light chain 2 (MLC-2) and $\alpha$-actinin observed in transverse arcs of a U2-OS cell.

(A) Single frame from a timelapse movie transiently expressing GFP-MLC2 and mCh- $\alpha$-actinin. Scale bar: $5 \mu \mathrm{m}$. 
Figure. 2: The semi-sarcomeric pattern of myosin light chain 2 (MLC-2) and $\alpha$ actinin observed in transverse arcs of a U2-OS cell.

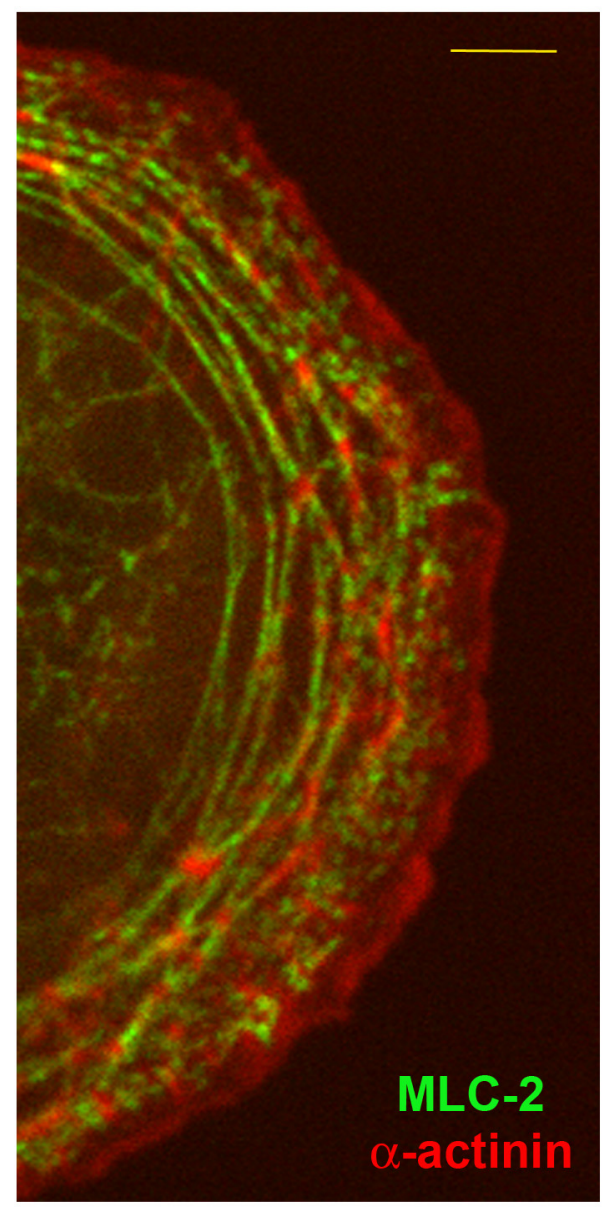


Lamellipodial and lamellar proteins relevant to the study

Lamellipodial and lamellar actin networks have distinct signature proteins associated with them. Because this study addresses changes in global actin network organization, the following section discusses relevant actin-binding proteins present in the lamellipodia and lamella whose association with actin networks could be influenced by dynamin2dependent actin remodeling.

\section{Cortactin}

Cortactin is an actin-binding and regulatory protein present at the lamellipodia. It binds and stabilizes Arp2/3-nucleated actin branches via its N-terminal acidic region (NTA) (Weaver et al., 2002; Weaver et al., 2001). As a result, cortactin depletion affects lamellipodial protrusions, due to the inability to stabilize these actin branches (Bryce et al., 2005). Cortactin interacts with dynamin via its SH3 domain, and dynamin's proline rich domain (PRD) (McNiven et al., 2000). It is therefore one of the primary candidates for mediating dynamin2-dependent effects on actin. A cortactin-dynamin complex has been implicated in a variety of actin-regulatory functions, both during endocytosis and non-endocytic processes (Chua et al., 2009; Mooren et al., 2009; Zhu et al., 2005). Cortactin is regulated via phosphorylation by serine/threonine and tyrosine kinases and also by acetylation, all of which extensively influence cortactin's functions in cell migration and F-actin interaction (MacGrath and Koleske, 2012). Phosphorylation of cortactin is observed in several metastatic cancers (MacGrath and Koleske, 2012). 


\section{Actin nucleating proteins}

Rapid actin polymerization at the leading edge is essential for cell migration. Because spontaneous association of actin monomers to initiate actin polymerization is kinetically unfavorable and hence the rate-limiting step, proteins that nucleate actin filaments are important for fast actin polymerization (Goley and Welch, 2006; Pollard and Borisy, 2003). The three major classes of actin nucleators are Arp2/3, formins and Spire. While formins and Spire nucleate unbranched actin filaments, Arp2/3 can bind to pointed ends of actin and nucleate filaments from the sides of pre-existing actin filaments, forming a branched actin network (Goley and Welch, 2006; Mullins et al., 1998; Pollard and Borisy, 2003).

Actin related protein 2/3(Arp2/3) complex is a seven-subunit complex that can nucleate actin filaments. The subunits Arp2 and Arp3 are structurally similar to actin and can mimic actin barbed ends, nucleating actin polymerization. The isolated Arp $2 / 3$ complex is inactive and it is activated to nucleate actin by nucleation promoting factors (NPFs) like Wiskott-Aldrich syndrome protein (WASP). The C-terminal of WASP binds Arp2/3 and activates it (Goley and Welch, 2006). Characteristic of its function as an actin nucleating and filament branching protein, Arp2/3 is present at the leading lamellipodial edge of migrating cells where a dendritic actin network forms, localizing at branch points as shown by immunogold labeling (Svitkina and Borisy, 1999). Even though localized at the lamellipodia, Arp2/3 is required for formation of lamellar transverse arcs in U2-OS cells, as siRNA-mediated depletion of the subunit p34 disrupts arc formation (Hotulainen and Lappalainen, 2006). 
The second class of nucleating proteins, formins, remains processively associated with actin-barbed ends following nucleation and during polymerization-induced actin filament elongation (Kovar, 2006). This association prevents binding of actin capping proteins to barbed ends and hence facilitates continued filament elongation at structures like filopodia and lamellar dorsal stress fibers (Hotulainen and Lappalainen, 2006; Kovar, 2006). As opposed to Arp2/3, formins nucleate unbranched filament polymerization. The third, and most recently discovered, class of actin nucleators are the Spire proteins. Spire has four actin-binding WH2 domains, which nucleate a string of four actin monomers. This four-monomer nucleus then initiates actin polymerization, with Spire remaining attached to the pointed end (Baum and Kunda, 2005; Quinlan et al., 2005). Like formins, Spire nucleates unbranched actin polymerization.

\section{$\underline{\alpha-A c t i n i n}$}

$\alpha$-Actinin is an actin crosslinking protein that is present in the lamellipodia and is also a component of the actomyosin. It forms antiparallel homodimers and can cross-link F-actin bundles as well as dendritic, branched actin networks, making it a highly flexible actin binding protein (Courson and Rock, 2010). Each monomer consists of an Nterminal head that contains the actin-binding domain, a central region with four spectrin repeats and a tail that is a $\mathrm{CaM}$ (Calmodulin) domain with two EF hands. There are four isoforms of $\alpha$-actinin. $\alpha$-Actinin 1 and 4 are the non-muscle isoforms and 2 and 3 are the muscle isoforms (Otey and Carpen, 2004; Sjoblom et al., 2008). Calcium binding, phosphorylation and phosphoinositides regulate binding of non-muscle $\alpha$-actinin to actin (Fraley et al., 2003; Izaguirre et al., 2001; Otey and Carpen, 2004). 
$\alpha$-Actinin-crosslinked anti-parallel actin networks start associating with myosin II at the lamellipodia-lamella interface and progressively get organized to form the actomyosin in the lamella (Hotulainen and Lappalainen, 2006). At the lamellipodialamella boundary, $\alpha$-actinin influences focal adhesion maturation by providing a structural template for their elongation (Choi et al., 2008; Oakes et al., 2012). $\alpha$-Actinin is present in close apposition with F-actin at adhesions and serves as a critical linker between the actin network and the substratum (Brown et al., 2006; Hu et al., 2007; Kanchanawong et al., 2010).

Non muscle myosin II (myosin II)

Myosin II is a large mechanoenzyme that binds to actin and uses energy from ATP hydrolysis to fulfill its functions as a motor protein. It is a hexamer with two essential light chains, two regulatory light chains and two heavy chains. The N-terminal region of each heavy chain consists of a globular head domain with binding sites for actin and ATP and a coiled-coiled region in the $\mathrm{C}$ terminus. The heavy chains dimerize and these dimers orient in an anti-parallel fashion to form bipolar myosin II filaments (Sellers, 2000; Vicente-Manzanares et al., 2009b) in the lamella.

One of the primary modes of regulation of myosin II is via phosphorylation. The regulatory light chain (RLC) is phosphorylated at Ser19 and Thr18 by myosin light chain kinase (MLCK), which increases the myosin II-ATPase activity (Levayer and Lecuit, 2012; Vicente-Manzanares et al., 2009b). RLC activity is also regulated by inhibition of myosin phosphatases, carried out by a downstream Rho GTPase effector ROCK. Phosphorylation of myosin II changes its conformation to a filament, promoting 
interactions with actin (Craig et al., 1983). Hence, myosin II light chain phosphorylation is a key regulator of actomyosin contractility.

\section{Localization of dynamin2 in U2OS cells}

To elucidate a function for dynamin2 in regulating actin networks in the lamellipodia and lamella, the first question to be addressed is where the protein localizes. In a live osteosarcoma cell line U2-OS, apart from putative endocytic structures and dynamic clathrin-coated pits (Fig. 3A, arrows), a separate pool of dynamin2 localizes to the distal edge of the lamellipodia (Fig. 3A). Furthermore, dynamin2 associates predominantly with advancing edges of protrusions (arrows, Fig. 3B). I hypothesize a function for dynamin2 in locally regulating lamellipodial actin networks along advancing edges of protrusions. 
Figure. 3: Dynamin2 localizes to the distal edge of the lamellipodia in live U2OS cells.

(A) Single frames from movies of dynamin2-depleted U2OS cells transiently expressing GFP-dyn2-WT and mCherry- $\alpha$-actinin. Dynamin2 clearly localizes to the distal lamellipodial edge, apart from at clathrin-coated pits and putative endosomal structures (arrows). Scale bar: $2 \mu \mathrm{m}$.

(B) Kymographs from movies of cells expressing GFP-dyn2-WT and mCh- $\alpha$-actinin. In many cells, dynamin2 localizes predominantly to advancing edges of protrusions (arrows). Scale bar: $1 \mu \mathrm{m}$. 
Figure. 3: Dynamin2 localizes to the distal edge of the lamellipodia in live U2OS cells.
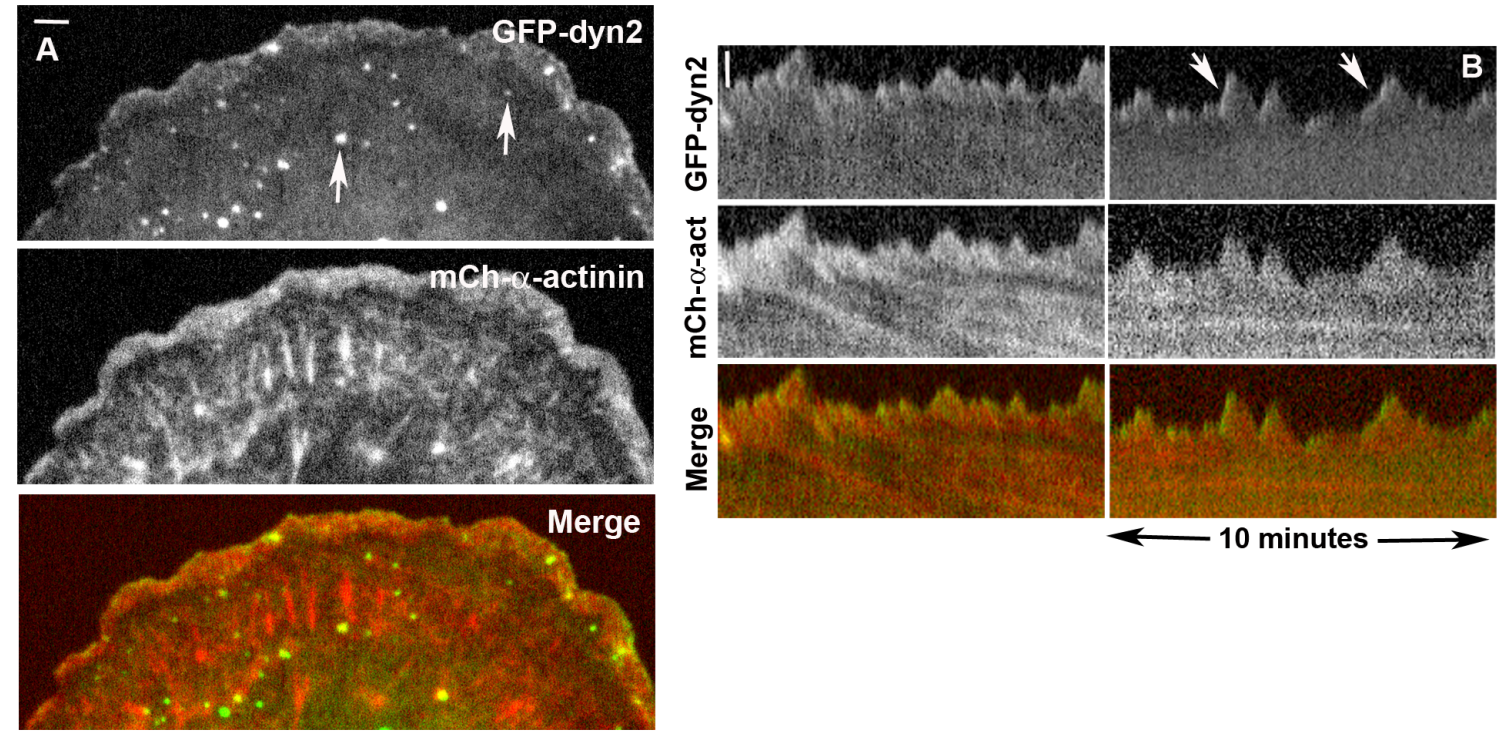


\section{Rationale for the study}

In this dissertation, I will address a novel role for dynamin2 in organization of lamellipodial and lamellar actin networks. Recent work has implicated dynamin2 in actomyosin organization and contractility (Chua et al., 2009; Mooren et al., 2009). Dynamin2 regulates apical actomyosin contractility in epithelial cells, via interactions with cortactin and influenced by Rho and myosin II (Chua et al., 2009). Using various GTP mutants of dynamin2, the authors postulated a function for dynamin2 in regulating apical contractility in a nucleotide dependent manner (Chua et al., 2009), which could be related to dynamin2's role as an actin remodeler. Dynamin2, in the presence of cortactin, regulates actin bundling and GTP hydrolysis-dependent actin reorganization in vitro and regulates actomyosin organization in vivo (Mooren et al., 2009). Dynamin2 depletion enhances the association of $\alpha$-actinin and myosin II with lamellar actin networks (Mooren et al., 2009), suggesting defects in network architecture. While these studies focused on dynamin2's interaction with cortactin, there is also evidence of dynamin1 directly binding to F-actin. An actin-binding deficient mutant of dynamin 1 affects stress fiber organization and actin polymerization in vivo (Gu et al., 2010). Therefore, dynamin is emerging as an important player in organizing actin networks. However, all these studies were performed on fixed cells and hence some of the dynamic aspects of actomyosin formation and flow could not be resolved. In my research, I address how dynamin2 influences actomyosin assembly and dynamics, using live U2-OS cells. Transient expression of fluorescently tagged dynamin2-WT and various mutants in live cells provide information on localization and vital clues as to how this protein functions in remodeling actin networks. 


\section{References}

Anderson, T.W., Vaughan, A.N., and Cramer, L.P. (2008). Retrograde flow and myosin II activity within the leading cell edge deliver F-actin to the lamella to seed the formation of graded polarity actomyosin II filament bundles in migrating fibroblasts. Mol Biol Cell 19, 5006-5018.

Bashkirov, P.V., Akimov, S.A., Evseev, A.I., Schmid, S.L., Zimmerberg, J., and Frolov, V.A. (2008). GTPase cycle of dynamin is coupled to membrane squeeze and release, leading to spontaneous fission. Cell 135, 1276-1286.

Baum, B., and Kunda, P. (2005). Actin nucleation: spire - actin nucleator in a class of its own. Curr Biol 15, R305-308.

Bernstein, B.W., and Bamburg, J.R. (2010). ADF/cofilin: a functional node in cell biology. Trends Cell Biol 20, 187-195.

Blanchoin, L., Amann, K.J., Higgs, H.N., Marchand, J.B., Kaiser, D.A., and Pollard, T.D. (2000). Direct observation of dendritic actin filament networks nucleated by Arp2/3 complex and WASP/Scar proteins. Nature 404, 1007-1011.

Brieher, W.M., Kueh, H.Y., Ballif, B.A., and Mitchison, T.J. (2006). Rapid actin monomer-insensitive depolymerization of Listeria actin comet tails by cofilin, coronin, and Aip1. J Cell Biol 175, 315-324.

Brown, C.M., Hebert, B., Kolin, D.L., Zareno, J., Whitmore, L., Horwitz, A.R., and Wiseman, P.W. (2006). Probing the integrin-actin linkage using high-resolution protein velocity mapping. J Cell Sci 119, 5204-5214.

Bryce, N.S., Clark, E.S., Leysath, J.L., Currie, J.D., Webb, D.J., and Weaver, A.M. (2005). Cortactin promotes cell motility by enhancing lamellipodial persistence. Curr Biol 15, 1276-1285.

Burnette, D.T., Manley, S., Sengupta, P., Sougrat, R., Davidson, M.W., Kachar, B., and Lippincott-Schwartz, J. (2011). A role for actin arcs in the leading-edge advance of migrating cells. Nat Cell Biol 13,371-381.

Cao, H., Garcia, F., and McNiven, M.A. (1998). Differential distribution of dynamin isoforms in mammalian cells. Mol Biol Cell 9, 2595-2609.

Chappie, J.S., Mears, J.A., Fang, S., Leonard, M., Schmid, S.L., Milligan, R.A., Hinshaw, J.E., and Dyda, F. (2011). A pseudoatomic model of the dynamin polymer identifies a hydrolysis-dependent powerstroke. Cell 147, 209-222. 
Choi, C.K., Vicente-Manzanares, M., Zareno, J., Whitmore, L.A., Mogilner, A., and Horwitz, A.R. (2008). Actin and alpha-actinin orchestrate the assembly and maturation of nascent adhesions in a myosin II motor-independent manner. Nat Cell Biol 10, 10391050 .

Chua, J., Rikhy, R., and Lippincott-Schwartz, J. (2009). Dynamin 2 orchestrates the global actomyosin cytoskeleton for epithelial maintenance and apical constriction. Proc Natl Acad Sci U S A 106, 20770-20775.

Cook, T., Mesa, K., and Urrutia, R. (1996). Three dynamin-encoding genes are differentially expressed in developing rat brain. J Neurochem 67, 927-931.

Cook, T.A., Urrutia, R., and McNiven, M.A. (1994). Identification of dynamin 2, an isoform ubiquitously expressed in rat tissues. Proc Natl Acad Sci U S A 91, 644-648.

Courson, D.S., and Rock, R.S. (2010). Actin cross-link assembly and disassembly mechanics for alpha-Actinin and fascin. J Biol Chem 285, 26350-26357.

Craig, R., Smith, R., and Kendrick-Jones, J. (1983). Light-chain phosphorylation controls the conformation of vertebrate non-muscle and smooth muscle myosin molecules. Nature $302,436-439$.

Ferguson, S.M., and De Camilli, P. (2012). Dynamin, a membrane-remodelling GTPase. Nat Rev Mol Cell Biol 13, 75-88.

Ferguson, S.M., Raimondi, A., Paradise, S., Shen, H., Mesaki, K., Ferguson, A., Destaing, O., Ko, G., Takasaki, J., Cremona, O., et al. (2009). Coordinated actions of actin and BAR proteins upstream of dynamin at endocytic clathrin-coated pits. Dev Cell $17,811-822$.

Fraley, T.S., Tran, T.C., Corgan, A.M., Nash, C.A., Hao, J., Critchley, D.R., and Greenwood, J.A. (2003). Phosphoinositide binding inhibits alpha-actinin bundling activity. J Biol Chem 278, 24039-24045.

Giannone, G., Mege, R.M., and Thoumine, O. (2009). Multi-level molecular clutches in motile cell processes. Trends Cell Biol 19, 475-486.

Goley, E.D., and Welch, M.D. (2006). The ARP2/3 complex: an actin nucleator comes of age. Nat Rev Mol Cell Biol 7, 713-726.

Gray, N.W., Kruchten, A.E., Chen, J., and McNiven, M.A. (2005). A dynamin-3 spliced variant modulates the actin/cortactin-dependent morphogenesis of dendritic spines. J Cell Sci $118,1279-1290$. 
Gu, C., Yaddanapudi, S., Weins, A., Osborn, T., Reiser, J., Pollak, M., Hartwig, J., and Sever, S. (2010). Direct dynamin-actin interactions regulate the actin cytoskeleton. Embo J 29, 3593-3606.

Hotulainen, P., and Lappalainen, P. (2006). Stress fibers are generated by two distinct actin assembly mechanisms in motile cells. J Cell Biol 173, 383-394.

Hu, K., Ji, L., Applegate, K.T., Danuser, G., and Waterman-Storer, C.M. (2007). Differential transmission of actin motion within focal adhesions. Science 315, 111-115.

Izaguirre, G., Aguirre, L., Hu, Y.P., Lee, H.Y., Schlaepfer, D.D., Aneskievich, B.J., and Haimovich, B. (2001). The cytoskeletal/non-muscle isoform of alpha-actinin is phosphorylated on its actin-binding domain by the focal adhesion kinase. J Biol Chem $276,28676-28685$.

Kanchanawong, P., Shtengel, G., Pasapera, A.M., Ramko, E.B., Davidson, M.W., Hess, H.F., and Waterman, C.M. (2010). Nanoscale architecture of integrin-based cell adhesions. Nature 468, 580-584.

Kovar, D.R. (2006). Molecular details of formin-mediated actin assembly. Curr Opin Cell Biol 18, 11-17.

Kruchten, A.E., and McNiven, M.A. (2006). Dynamin as a mover and pincher during cell migration and invasion. J Cell Sci 119, 1683-1690.

Lee, E., and De Camilli, P. (2002). Dynamin at actin tails. Proc Natl Acad Sci U S A 99, 161-166.

Levayer, R., and Lecuit, T. (2012). Biomechanical regulation of contractility: spatial control and dynamics. Trends Cell Biol 22, 61-81.

Liu, Y.W., Neumann, S., Ramachandran, R., Ferguson, S.M., Pucadyil, T.J., and Schmid, S.L. (2011). Differential curvature sensing and generating activities of dynamin isoforms provide opportunities for tissue-specific regulation. Proc Natl Acad Sci U S A 108, E234242.

Liu, Y.W., Surka, M.C., Schroeter, T., Lukiyanchuk, V., and Schmid, S.L. (2008). Isoform and splice-variant specific functions of dynamin-2 revealed by analysis of conditional knock-out cells. Mol Biol Cell 19, 5347-5359.

MacGrath, S.M., and Koleske, A.J. (2012). Cortactin in cell migration and cancer at a glance. J Cell Sci 125, 1621-1626.

McNiven, M.A., Kim, L., Krueger, E.W., Orth, J.D., Cao, H., and Wong, T.W. (2000). Regulated interactions between dynamin and the actin-binding protein cortactin modulate cell shape. J Cell Biol 151, 187-198. 
Menon, M., and Schafer, D.A. (2013). Dynamin: expanding its scope to the cytoskeleton. International Review of Cell and Molecular Biology 302, 188-219.

Mooren, O.L., Kotova, T.I., Moore, A.J., and Schafer, D.A. (2009). Dynamin2 GTPase and cortactin remodel actin filaments. J Biol Chem 284, 23995-24005.

Mullins, R.D., Heuser, J.A., and Pollard, T.D. (1998). The interaction of Arp2/3 complex with actin: nucleation, high affinity pointed end capping, and formation of branching networks of filaments. Proc Natl Acad Sci U S A 95, 6181-6186.

Nakata, T., Takemura, R., and Hirokawa, N. (1993). A novel member of the dynamin family of GTP-binding proteins is expressed specifically in the testis. J Cell Sci 105 ( Pt 1), 1-5.

Nemethova, M., Auinger, S., and Small, J.V. (2008). Building the actin cytoskeleton: filopodia contribute to the construction of contractile bundles in the lamella. J Cell Biol $180,1233-1244$.

Oakes, P.W., Beckham, Y., Stricker, J., and Gardel, M.L. (2012). Tension is required but not sufficient for focal adhesion maturation without a stress fiber template. J Cell Biol 196, 363-374.

Orth, J.D., Krueger, E.W., Cao, H., and McNiven, M.A. (2002). The large GTPase dynamin regulates actin comet formation and movement in living cells. Proc Natl Acad Sci U S A 99, 167-172.

Otey, C.A., and Carpen, O. (2004). Alpha-actinin revisited: a fresh look at an old player. Cell Motil Cytoskeleton 58, 104-111.

Pollard, T.D., and Borisy, G.G. (2003). Cellular motility driven by assembly and disassembly of actin filaments. Cell 112, 453-465.

Ponti, A., Machacek, M., Gupton, S.L., Waterman-Storer, C.M., and Danuser, G. (2004). Two distinct actin networks drive the protrusion of migrating cells. Science 305, 17821786.

Praefcke, G.J., and McMahon, H.T. (2004). The dynamin superfamily: universal membrane tubulation and fission molecules? Nat Rev Mol Cell Biol 5, 133-147.

Quinlan, M.E., Heuser, J.E., Kerkhoff, E., and Mullins, R.D. (2005). Drosophila Spire is an actin nucleation factor. Nature 433, 382-388.

Schwarz, U.S., and Gardel, M.L. (2012). United we stand: integrating the actin cytoskeleton and cell-matrix adhesions in cellular mechanotransduction. J Cell Sci 125, 3051-3060. 
Sellers, J.R. (2000). Myosins: a diverse superfamily. Biochim Biophys Acta 1496, 3-22.

Shutova, M., Yang, C., Vasiliev, J.M., and Svitkina, T. (2012). Functions of nonmuscle myosin II in assembly of the cellular contractile system. PLoS One 7, e40814.

Sjoblom, B., Salmazo, A., and Djinovic-Carugo, K. (2008). Alpha-actinin structure and regulation. Cell Mol Life Sci 65, 2688-2701.

Sontag, J.M., Fykse, E.M., Ushkaryov, Y., Liu, J.P., Robinson, P.J., and Sudhof, T.C. (1994). Differential expression and regulation of multiple dynamins. J Biol Chem 269, 4547-4554.

Svitkina, T. (2007). Electron microscopic analysis of the leading edge in migrating cells. Methods Cell Biol 79, 295-319.

Svitkina, T.M., and Borisy, G.G. (1999). Arp2/3 complex and actin depolymerizing factor cofilin in dendritic organization and treadmilling of actin filament array in lamellipodia. Journal of Cell Biology 145, 1009-1026.

Tojkander, S., Gateva, G., and Lappalainen, P. (2012). Actin stress fibers--assembly, dynamics and biological roles. J Cell Sci 125, 1855-1864.

Urrutia, R., Henley, J.R., Cook, T., and McNiven, M.A. (1997). The dynamins: redundant or distinct functions for an expanding family of related GTPases? Proc Natl Acad Sci U S A 94,377-384.

Vallotton, P., and Small, J.V. (2009). Shifting views on the leading role of the lamellipodium in cell migration: speckle tracking revisited. J Cell Sci 122, 1955-1958.

Van Troys, M., Huyck, L., Leyman, S., Dhaese, S., Vandekerkhove, J., and Ampe, C. (2008). Ins and outs of ADF/cofilin activity and regulation. Eur J Cell Biol 87, 649-667.

Verkhovsky, A.B., Svitkina, T.M., and Borisy, G.G. (1995). Myosin II filament assemblies in the active lamella of fibroblasts: their morphogenesis and role in the formation of actin filament bundles. J Cell Biol 131, 989-1002.

Vicente-Manzanares, M., Choi, C.K., and Horwitz, A.R. (2009a). Integrins in cell migration--the actin connection. J Cell Sci 122, 199-206.

Vicente-Manzanares, M., Ma, X., Adelstein, R.S., and Horwitz, A.R. (2009b). Nonmuscle myosin II takes centre stage in cell adhesion and migration. Nat Rev Mol Cell Biol 10, 778-790. 
Weaver, A.M., Heuser, J.E., Karginov, A.V., Lee, W.L., Parsons, J.T., and Cooper, J.A. (2002). Interaction of cortactin and N-WASp with Arp2/3 complex. Curr Biol 12, 12701278.

Weaver, A.M., Karginov, A.V., Kinley, A.W., Weed, S.A., Li, Y., Parsons, J.T., and Cooper, J.A. (2001). Cortactin promotes and stabilizes Arp2/3-induced actin filament network formation. Curr Biol 11, 370-374.

Zhu, J., Zhou, K., Hao, J.J., Liu, J., Smith, N., and Zhan, X. (2005). Regulation of cortactin/dynamin interaction by actin polymerization during the fission of clathrincoated pits. J Cell Sci 118, 807-817. 


\section{Chapter 2}

\section{Dynamin: expanding its scope to the cytoskeleton}

This chapter is based on previously published work.

Menon M, Schafer DA. Dynamin: expanding its scope to the cytoskeleton

Int Rev Cell Mol Biol. 2013;302:187-219. doi: 10.1016/B978-0-12-407699-0.00003-0. 


\begin{abstract}
$\underline{\text { Abstract }}$
The large GTPase dynamin is well known for its actions on budded cellular membranes to generate vesicles, most often, clathrin-coated endocytic vesicles. The scope of cellular processes in which dynamin-mediated vesicle formation occurs has expanded to include secretory vesicle formation at the Golgi, from other endosomes and from non-clathrin structures, such as caveolae, as well as membrane remodeling during exocytosis and vesicle fusion. An intriguing new facet of dynamin's sphere of influence is the cytoskeleton. Cytoskeletal filament networks maintain cell shape, provide cell movement, execute cell division and orchestrate vesicle trafficking. Recent evidence supports the hypothesis that dynamin influences actin filaments and microtubules via mechanisms that are independent of its membrane remodeling activities. In this review, we discuss this emerging evidence and consider possible mechanisms of action.
\end{abstract}




\section{Introduction}

The cytoskeleton forms a dynamic structural framework that works in concert with cellular membranes and the extracellular matrix to maintain cell shape and tissue integrity, execute migration, morphogenesis and cell division, and orchestrate protein and membrane trafficking. Several hundred proteins regulate the dynamic interactions of cytoskeletal filaments and membranes during fundamental cellular processes. The large GTPase dynamin, which is best known for its activities that remodel membranes, is emerging as a regulator of cytoskeletal filaments. Whereas dynamin is soundly established to generate vesicles during endocytosis (Doherty and McMahon, 2009; Ferguson and De Camilli, 2012; Praefcke and McMahon, 2004; Ramachandran, 2011; Schmid and Frolov, 2011), its original discovery as a microtubule binding protein (Shpetner and Vallee, 1989), together with early observations of defective neurite extension and growth cone migration by cultured neurons from Drosophila carrying a defective dynamin gene (Kim and $\mathrm{Wu}, 1987$ ), provided early hints that dynamins may also influence processes that depend on cytoskeletal filaments. Over subsequent decades, insight into the abilities of dynamin to influence the dynamics of select actin filament networks and microtubules, and of its possible molecular mechanisms of action, have emerged. In this review, we highlight those actin- and microtubule-dependent processes in which a regulatory role for dynamin is supported. An outstanding challenge is to demonstrate unequivocally that dynamin influences cytoskeletal networks independently from its well-characterized functions in endocytosis. To provide a contextual framework, we begin with a succinct review of the membrane remodeling activities of dynamin during endocytosis and exocytosis. 


\section{Dynamin - a Membrane Remodeling GTPase}

Endocytosis is the process whereby contents of the plasma membrane and extracellular fluids are delivered to the intracellular milieu via vesicles formed at the plasma membrane (Conner and Schmid, 2003; Doherty and McMahon, 2009). Endocytosis occurs by many different mechanisms, including clathrin-mediated endocytosis, clathrin-independent endocytosis, macropinocytosis, phagocytosis and caveolin-dependent endocytosis. Of these many different routes, the mechanisms by which clathrin mediates endocytosis is best understood. During clathrin-mediated endocytosis (CME), adaptor proteins bind and cluster membrane receptors and, together with assembled clathrin lattices, initiate the inward budding of the plasma membrane; as the membrane invaginates more deeply it remains tethered via a thin, tubular membrane neck. Finally, dynamin-mediated scission of the thin, tubular tether releases a vesicle containing the internalized membrane and fluid components to the cytoplasm (Doherty and McMahon, 2009; Ferguson and De Camilli, 2012; McMahon and Boucrot, 2011; Schmid and Frolov, 2011).

Several non-mutually exclusive models for the mechanisms by which dynamin catalyzes scission have been proposed. A common feature of all models is the assembly of dynamin as a helical polymer around the thin, tubular necks that tether deeply invaginated clathrin-coated pits to the plasma membrane. Some models invoke GTP hydrolysis-dependent conformational changes within the assembled dynamin polymer that mechanically constricts, extends or twists the membrane necks, leading to scission and vesicle formation (Danino et al., 2004; Gu et al., 2010; Roux et al., 2006; Stowell et 
al., 1999). Recent evidence from structural studies supports the idea that a mechanochemical power stroke may be involved (Chappie et al., 2011). Another model suggests that GTP hydrolysis-dependent cycles of assembly and disassembly of dynamin oligomers on thin necks subsequently leads to membrane scission, perhaps by locally destabilizing structure at membrane interfaces of the neck (Bashkirov et al., 2008; Pucadyil and Schmid, 2008). An outstanding question is how GTP hydrolysis, the associated conformational changes within the dynamin collar or its cycles of assembly and disassembly are linked to membrane scission.

More recently, dynamin was implicated in membrane remodeling during exocytosis (Anantharam et al., 2011; Fulop et al., 2008; Gonzalez-Jamett et al., 2010; Holroyd et al., 2002; Jaiswal et al., 2009). During chromaffin granule secretion, the rate of dynamin GTPase activity controlled the rate of fusion pore expansion and thereby, regulated the kinetics and amount of released secretory vesicle content (Anantharam et al., 2011). Mutant dynamin with an accelerated rate of GTP hydrolysis increased the rate of fusion pore expansion and the frequency of full fusion with the plasma membrane, whereas mutant dynamin with decreased rates of GTP hydrolysis stabilized fusion pores and delayed expansion to full fusion. How the GTPase cycle modulates fusion pore expansion is not known. The GTPase cycle may control interactions of dynamin with other components of the fusion machinery, such as synaptophysin (Gonzalez-Jamett et al., 2010). Alternatively, assembled dynamin oligomers may restrict fusion pore expansion with cycles of GTP hydrolysis regulating the half-life of assembled dynamin at the fusion pore and, thereby, fusion pore dynamics (Anantharam et al., 2011). The cortical actin network also modulates fusion pore dynamics (Jaiswal et al., 2009) and a 
function for dynamin in regulating actin filaments at sites of exocytosis has not been ruled out. The ability of dynamin to regulate fusion pores during exocytosis expands the repertoire of cellular processes influenced by this versatile, membrane-remodeling GTPase.

\subsection{Dynamin structure and biochemistry}

Dynamin is a large GTPase composed of $100 \mathrm{kDa}$ subunits that associate as tetramers. Tetrameric dynamin further oligomerizes to form a helical polymer around tubular templates, such as the necks of deeply invaginated clathrin-coated pits. Interestingly, dynamin also forms helical polymers around other structures with the appropriate electrostatic charge and diameter, such as microtubules and bundles of actin filaments (Gu et al., 2010; Shpetner and Vallee, 1989). Dynamin also assembles as stable ring-like structures in the absence of tubular templates (Hinshaw and Schmid, 1995). Recent X-ray crystallographic studies of nearly full-length dynamin1 revealed a dimeric structure composed of two extended, anti-parallel dynamin polypeptides with a subunitsubunit interface situated near the base of the central "stalk" domain and the globular, Nterminal GTP-binding G-domains oriented at opposite ends of the extended dimer (Faelber et al., 2011; Ford et al., 2011). A pleckstrin homology (PH) domain was situated in a foot-like globular structure at the base of each stalk, creating the membranebinding face of the extended dimer. Not yet examined is the structure of the C-terminal proline-rich domain (PRD) of dynamin, however, it is expected to project toward the Gdomain surface, opposite the membrane-binding surface. Through these major domains (G-domain, stalk, PH domain and PRD), dynamin executes several activities, including 
hydrolysis of GTP, self-assembly via interactions between dynamin stalk regions, binding to anionic lipids via the $\mathrm{PH}$ domain, and interactions with a growing list of proteins that bind primarily via the PRD. Each of these domains and activities is required for vesicle scission by dynamin.

\subsubsection{The dynamin GTPase cycle}

Unassembled dynamin binds GTP with low affinity and exhibits a low basal rate of GTP hydrolysis that is stimulated upon self-assembly as oligomers (Stowell et al., 1999; Warnock and Schmid, 1996). Recent biochemical and structural studies indicated that assembly-stimulated GTP hydrolysis results from interactions of G-domains between adjacent rungs of the helical dynamin polymer assembled around a tubular template (Chappie et al., 2010; Chappie et al., 2011). Several PRD-interacting proteins, including Grb2 (Barylko et al., 1998), cortactin (Mooren et al., 2009) and SNX9 (Soulet et al., 2005), also stimulate the basal rate of GTP hydrolysis by dynamin.

The importance of GTP hydrolysis by dynamin for membrane remodeling was first highlighted by electron microscopic images of synaptic membrane preparations incubated in the presence of GTP $\gamma$ S, a non-hydrolyzable GTP analog (Takei et al., 1995). Elongated membrane tubules decorated by electron-dense ring-like structures formed in such preparations; the tubules also labeled with anti-dynamin antibodies. Subsequent characterization of mutant dynamin proteins defective in either GTP binding, the GTP hydrolysis catalytic rate or assembly-stimulated GTPase activity supported the hypothesis that GTP hydrolysis by dynamin powered membrane scission during CME (Marks et al., 2001; Song et al., 2004a; Song et al., 2004b). Cryo-electron microscopy of dynamin 
assembled on lipid tubules suggested that GTP hydrolysis-dependent conformational changes within the assembled dynamin polymer were coupled to constriction of membrane tubules, leading ultimately to membrane scission (Gu et al., 2010; Hinshaw and Schmid, 1995). Indeed, recent structures of a dynamin1 fusion protein obtained in the presence of either non-hydrolyzable or transition state guanine nucleotides provides evidence of a GTPase-dependent powerstroke (Chappie et al., 2009; Chappie et al., 2011). Whether the putative powerstroke exerts forces that drive scission is not yet known.

\subsubsection{Dynamin's pleckstrin homology $(\mathrm{PH})$ domain mediates interactions with}

\section{phospholipids}

Positively charged amino acids within the dynamin PH domain mediate binding to acidic phospholipids, particularly phosphoinositides (Ferguson et al., 1994; Zheng et al., 1996). While the affinity of a single PH domain for anionic lipids is low, cooperative binding by oligomeric dynamin increases the avidity (Klein et al., 1998). Additionally, the PH domain appears to play an active role in vesicle scission. Dynamin binding to liposomes was coupled to partial insertion into the membrane of a hydrophobic variable loop, VL1, of the PH domain (Ramachandran et al., 2009; Ramachandran and Schmid, 2008). Membrane insertion by VL1 may sense or generate membrane curvature along tubular membranes or destabilize bilayer structure to promote membrane scission (Bethoney et al., 2009; Ramachandran et al., 2009). Whereas the primary structure of the $\mathrm{PH}$ domains of dynamin1 and dynamin2 are quite similar, these dynamin isoforms exhibit distinct abilities to assemble and generate curvature on lipid templates (Liu et al., 
2011b). The differential activities of dynamin1 and dynamin 2 were attributed to a single amino acid residue in a different variable loop, VL3, of the PH domain. Switching one amino acid in dynamin2 VL3 to tyrosine, which exists at that position in dynamin1, was sufficient to confer dynamin1-like membrane scission and curvature-generating properties to dynamin2 (Liu et al., 2011b). In neurons, dynamin1 drives rapid recycling of synaptic vesicle membranes, whereas dynamin2 is the workhorse for slow endocytic events that require clustering of membrane receptors, adaptor proteins and the assembly of clathrin lattices. Thus, distinct modes of membrane remodeling may be governed by differential interactions of dynamin isoforms with membranes.

\subsubsection{The C-terminal, proline-rich domain (PRD)}

More than 30 proteins interact directly with dynamin, the majority of which bind the C-terminal PRD (Ferguson and De Camilli, 2012; Schafer, 2004). The PRD contains several PXXP motifs that interact with SH3-domain-containing proteins, but the specific binding sites within the PRD for most dynamin binding partners have, for the most part, not been determined. During endocytosis, the PRD targets dynamin to clathrin-coated structures through interactions with amphiphysin and endophilin (David et al., 1996; Ringstad et al., 1999; Sundborger et al., 2011; Takei et al., 1999). Dynamin is targeted to select F-actin networks via PRD-mediated interactions with cortactin or Abp1 (Kessels et al., 2001; McNiven et al., 2000). Interestingly, a region of the PRD restricts dynamin interactions with microtubules during interphase, but phosphorylation within the PRD by mitotic kinase activity permits dynamin interactions with microtubules during mitosis (Morita et al., 2010; Shpetner et al., 1996). Thus, given such a diverse array of PRD 
interacting partners and coupled with GTPase-regulated self-assembly, dynamin is positioned to function as a multi-dimensional scaffold capable of acting in different cellular processes through a complex array of dynamic protein-protein interactions. Identifying how these interactions are regulated in time and space to govern different dynamin-dependent functions remains an important goal.

\subsection{Experimental approaches to explore novel dynamin functions.}

Inhibiting dynamin has proven a useful strategy to explore its contributions and mechanisms of action during cellular processes, particularly endocytosis. Several mutant dynamin proteins that negatively impact membrane receptor internalization have been characterized, including those that alter GTP binding and/or hydrolysis, block selfassembly as oligomers and decrease interactions with phospholipids or interacting proteins. A common tool used to disrupt dynamin-dependent endocytosis is dynaminK44A, a mutant defective in binding GTP (Damke et al., 1994). Since dynamin-K44A potently blocks endocytosis, identifying cytoskeletal-related functions for dynamin is challenged if decreased endocytic activity impacts surface receptors or signaling pathways that impinge the cytoskeleton. Moreover, gain-of-function effects of dynaminK44A on actin filaments, such as actin filament crosslinking (Mooren et al., 2009), further confound interpretations of dynamin actions on cytoskeletal filaments. Overexpressed dynamin proteins may indirectly perturb cellular processes by sequestering dynamin binding partners or inducing cytotoxic effects (Liu et al., 2011a). Thus, strategies that monitor endocytosis activity and control the level of dynamin 
protein expression must be employed when using exogenously expressed dynamin proteins.

Genetic ablation or "knock out" of dynamin genes in mice, or approaches that conditionally deplete dynamin genes within specific cells or tissues can reveal roles for dynamin in cellular processes (Ferguson et al., 2009; Liu et al., 2008). Transient, RNAimediated knock-down typically achieves up to $90 \%$ depletion of dynamin proteins (Ezratty et al., 2005; Mooren et al., 2009). As with some mutant dynamin proteins, discerning novel functions for dynamin using gene knock-out or siRNA-mediated depletion is complicated if endocytosis is grossly perturbed. Thus, quantitative assessment of endocytic activity and of potential downstream signals should be carried out in concert with studies of novel functions for dynamin. However, when coupled with experiments in which expressed mutant dynamin proteins selectively restore endocytosis but not other processes, knock-down or genetic ablation approaches may reveal novel functions for dynamin.

New pharmacological agents that acutely block GTP hydrolysis by dynamin are frequently used to disrupt endocytosis (Macia et al., 2006; Yamada et al., 2009). However, the specificity, mechanism of action and selectivity of the available dynaminblocking drugs is not sufficiently characterized to be useful probes of novel, endocytosisindependent activities for dynamins. Finally, injected dynamin antibodies were used to inhibit dynamin function in some studies (Henley et al., 1998; Jones et al., 1998; Thompson et al., 2004).

\section{Actions of Dynamin and Actin Filaments during Endocytosis}


Both dynamin and actin filaments support CME and other forms of endocytosis in mammalian cells, including phagocytosis, macropinocytosis and caveolae-mediated endocytosis (Di et al., 2003; Galletta et al., 2010; Gold et al., 1999; Henley et al., 1998; Kaksonen et al., 2006; Lamaze et al., 1997; Lanzetti, 2007; Liu et al., 2008; Merrifield et al., 1999; Mooren et al., 2012; Schlunck et al., 2004; Shajahan et al., 2004; Tse et al., 2003). In contrast, in yeast, actin filaments together with other factors that induce membrane curvature execute vesicle scission for endocytosis (Aghamohammadzadeh and Ayscough, 2009; Kaksonen et al., 2003; Mooren et al., 2012; Weinberg and Drubin, 2012). In mammalian cells, actin filaments facilitate vesicle scission but are not absolutely required (Ferguson et al., 2009; Itoh et al., 2005; Taylor et al., 2012). Actin filaments were also implicated in supporting other steps of the endocytic process, including membrane invagination, formation or stabilization of deeply invaginated clathrin-coated stuctures or movement of newly-formed vesicles away from the plasma membrane (Girao et al., 2008; Liu et al., 2010; Mooren et al., 2012; Qualmann et al., 2000). Before considering how dynamin regulates cytoskeletal filaments outside the realm of endocytosis, we discuss emerging evidence supporting interdependent actions of dynamin and actin filaments during CME.

Both actin filaments and dynamin are recruited to clathrin-coated structures (CCS) at the plasma membrane. Low amounts of dynamin, but little actin, were associated with nascent CCS when they first form (Merrifield et al., 2002). Just prior to scission, dynamin accumulated rapidly at CCS; recruitment of actin, presumably from de novo assembly, preceded the rapid burst of dynamin recruitment and scission by $\sim 20$ seconds (Taylor et al., 2012). Factors that promote actin assembly (Arp2/3 complex, N- 
WASP and cortactin) were generally recruited to CCS prior to the scission event, whereas factors that promote F-actin disassembly (cofilin and coronin) appeared later, suggesting that F-actin turnover at clathrin-coated structures is temporally coupled to vesicle scission (Taylor et al., 2012).

A role for dynamin in F-actin turnover at CCS was also supported from studies of fibroblasts from conditional dynamin $1 /$ dynamin 2 double knockout mice. F-actin was highly enriched at deeply invaginated CCS in fibroblasts lacking both dynamin isoforms (Ferguson et al., 2009). Arp2/3 complex, an actin-nucleating factor that induces actin assembly (Pollard, 2007), was also enriched at CCS, indicating that dynamin was not required to recruit or activate the machinery for F-actin assembly. In contrast, dynamin likely functions to limit actin assembly or promote its turnover at CCS (Figure 1). The transient appearance of small patches of dendritic filament networks closely associated with invaginated CCS is consistent with this idea (Collins et al., 2011).

Dynamin and actin appear to function interdependently at CCS to power vesicle scission. Disrupting the actin cytoskeleton in cells lacking dynamin with pharmacologic agents increased the number of shallow, wide-necked, CCS at the expense of deeply invaginated CCS, supporting a role for actin filaments in membrane invagination and in stabilizing the thin necks that link CCS to membranes (Ferguson et al., 2009). Moreover, an intact actin cytoskeleton greatly enhanced dynamin recruitment and the efficiency of scission (Taylor et al., 2012). Thus, actin filaments facilitate endocytosis by stabilizing invaginated membranes, promoting the formation of deeply invaginated CCS with thin tubular necks and reinforcing recruitment of dynamin. The thin tubular necks may be ideal templates to assembly of dynamin collars. Actin filaments also could provide 
additional binding sites for dynamin at CCS via direct interactions (Gu et al., 2010) or via indirect interactions with the F-actin- and dynamin-binding proteins, cortactin or Abp1 (Kaksonen et al., 2000; Merrifield et al., 2005; Zhu et al., 2005). Whether or not dynamin influences actin filament turnover or filament organization for efficient vesicle formation remains to be explored.

Whereas actin filaments facilitated dynamin recruitment at CCS, the dynamin GTPase cycle also influenced F-actin assembly and disassembly as CCS, lending additional support for interdependent roles for dynamin and actin filaments during endocytosis (Taylor et al., 2012). Mutant dynamin proteins with impaired rates of GTP hydrolysis decreased the rate of actin recruitment and increased the rate of F-actin disassembly at CCS (Taylor et al., 2012). However, mutant dynamin proteins with increased rates of GTP hydrolysis had no effect on actin dynamics at CCS, suggesting that a threshold level of GTP hydrolysis by dynamin was sufficient to influence actin filaments.

Dynamin also may catalyze scission in coordination with actin filaments during some clathrin-independent processes. The cytokine receptor $\gamma \mathrm{c}$ is internalized via a clathrin-independent pathway that requires dynamin 2 and its binding partner, cortactin, which also binds actin filaments (Sauvonnet et al., 2005). As will be discussed in detail below, cortactin may be an important biochemical link between dynamin and actin filament networks for endocytosis and other cellular processes.

Phagocytosis, an endocytic process in which cells engulf particles, depends on Factin-rich protrusions of the plasma membrane. In cells in which dynamin2 was perturbed either by injecting anti-dynamin antibodies or by expressing mutant dynamin2- 
$\mathrm{K} 44 \mathrm{~A}$, actin-rich protrusions extended around internalizing particles, but the protrusions arrested before engulfment was complete (Di et al., 2003; Gold et al., 1999; Tse et al., 2003). Thus, dynamin 2 may remodel actin filaments within the protrusive structures to complete phagosome formation (Figure 1). Similar to phagocytosis, internalization of extracellular fluid via macropinocytosis also depends on dynamic actin filaments that support sheet-like ruffles that collapse rearward to encapsulate extracellular fluid (Kerr and Teasdale, 2009; Lim and Gleeson, 2011) and on dynamin (Liu et al., 2008; Schlunck et al., 2004). Whether or not dynamin remodels membranes, modulates actin filaments, or both, during macropinocytosis and the late stages of phagocytosis has not been examined.

In summary, considerable evidence supports the view that dynamin and actin filaments play interdependent roles during clathrin-dependent and clathrin-independent endocytic processes in mammalian cells. Although not universally required for CME, a functional actin cytoskeleton promotes formation and stability of deeply invaginated clathrin-coated pits and facilitates efficient vesicle scission. Cortactin, which interacts with actin filaments and with dynamin, is likely to link dynamin and actin filaments during many forms of endocytosis. However, other dynamin-interacting proteins may coordinate the concerted actions of dynamin and actin filaments in a site-specific manner.

\section{Beyond Endocytosis: Dynamin as a Cytoskeletal Regulator}

Dynamin was first discovered as a nucleotide-sensitive microtubule binding and bundling protein (Shpetner and Vallee, 1989). Dynamin (or in Greek, force or power) was so named based on speculations that it generated nucleotide hydrolysis-dependent 
force-producing interactions between bundled microtubules. Subsequent work showed dynamin hydrolyzed GTP, an activity that was stimulated by microtubules (Maeda et al., 1992; Shpetner and Vallee, 1992). When dynamin was identified as the product of the shibire gene in Drosophila melanogaster (Chen et al., 1991; van der Bliek and Meyerowitz, 1991), together with observations that dynamin decorated thin membrane tubules with clathrin-capped tips in GTP $\gamma \mathrm{S}$-treated synaptic membrane preparations (Takei et al., 1995), its destiny as a key regulator of vesicle scission was solidified. Understanding alternate functions and mechanisms of action of dynamin, particularly those that involve cytoskeletal dynamics, only recently returned to the forefront. In the sections that follow, we discuss evidence that implicates dynamin in regulating the actin and microtubule cytoskeletons.

\subsection{Dynamin and microtubules}

Since its discovery as a microtubule binding protein, dynamin was implicated in several microtubule-dependent processes in animal cells, including efficient mitosis, maintenance of centrosome structure and for microtubule dynamic instability (Tanabe and Takei, 2009; Thompson et al., 2004; Thompson et al., 2002). Dynamin did not localize with microtubules in interphase cells, but was localized with $\gamma$-tubulin of interphase and mitotic centrosomes (Chircop et al., 2011; Thompson et al., 2004). Dynamin was strikingly enriched along the bundled microtubules of the mid-zone spindle and mid-body during late stages of mitosis and cytokinesis (Hamao et al., 2009; Thompson et al., 2002). Although early events in mitosis and mid-zone microtubules appeared normal in mitotic fibroblastoid cells lacking dynamin2, the time required to 
complete cytokinesis was longer compared with control cells or with dynamin2-depleted cells expressing either dynamin1 or dynamin2 (Liu et al., 2008). Similarly, decreasing the expression of the single dynamin protein expressed in C. elegans or activating a temperature-sensitive mutant dynamin, blocked cytokinesis at a late stage (Thompson et al., 2002). Whether or not increased genomic instability results from decreased levels of endogenous dynamin 2 remains to be explored. Taken together, these findings indicate that dynamin acts directly on bundled microtubules of the mid-body to promote cell division. Depleting dynamin2 also caused individual centrosomes to separate by as much as 3 microns, without apparent gross perturbations to overall microtubule organization (Thompson et al., 2004). Thus, dynamin2 maintains centrosome cohesion in interphase cells but how this activity contributes to microtubule organization, polarized morphology or cell migration is not known.

The dynamin PRD negatively regulates interactions of dynamin with microtubules. A mutant form of dynamin lacking the C-terminal portion of the PRD strongly decorated microtubules of interphase cells and conferred resistance to coldinduced microtubule disassembly (Hamao et al., 2009). During mitosis, association of dynamin with mid-zone microtubules required phosphorylation within the PRD by cyclin-dependent kinase1, which presumably overrides the intrinsic block for binding microtubules (Chircop et al., 2011; Morita et al., 2010). The actions of dynamin on interphase centrosomes and on mitotic microtubules provides evidence supporting a bona fide physiologic function for dynamin in regulating select microtubule-dependent processes (Ishida et al., 2011; Thompson et al., 2004). 
Investigations of interphase cells depleted of dynamin2 revealed increased numbers of stable microtubules containing high levels of acetylated tubulin, a marker of stable microtubules (Figure 1) (Henmi et al., 2011; Tanabe and Takei, 2009). The increased stable microtubules were coupled to defects in transport of Golgi-derived vesicles, perhaps as a result of inefficient vesicle capture by stable, as opposed to dynamic, microtubules (Tanabe and Takei, 2009). Thus, through its effects on microtubule dynamics, dynamin2 may indirectly influence vesicle transport at the Golgi and mid-body. Stable microtubules in dynamin2-depleted cells may also negatively impact cell migration through effects on focal adhesion disassembly (Kaverina et al., 1999)

\subsection{Links between dynamin and F-actin networks}

Identifying functional links between dynamin and the actin cytoskeleton is an area of intense investigation. Early evidence implicating dynamin in actin-related functions came from studies of neurons from Drosophila melanogaster carrying temperaturesensitive mutations in the shibire gene, which encodes fly dynamin. shibire flies become paralyzed at restrictive temperatures due to defects in synaptic transmission (Kim and Wu, 1987; Poodry and Edgar, 1979; van der Bliek and Meyerowitz, 1991). Cultured neurons from shibire flies also exhibit defective neuritogenesis at restrictive temperatures, including stunted neurite extension and decreased growth cone motility (Kim and Wu, 1987). Subsequent studies have implicated dynamin in other actindependent processes, including cell migration (Kruchten and McNiven, 2006), invasion through extracellular matrix (Baldassarre et al., 2003), apical constriction in epithelial 
cells (Chua et al., 2009) and select forms of actin-dependent bacterial pathogeneis (Lee and De Camilli, 2002; Orth et al., 2002; Unsworth et al., 2007). Because dynamin action in endocytosis may influence signaling pathways that impinge on actin filaments, clarifying the relationship between dynamin regulation of actin filament networks and its endocytic functions is the primary challenge toward gaining a full understanding of the scope of dynamin activities. In the following section, we discuss emerging evidence supporting the hypothesis that dynamin is a multi-functional protein that directly influences actin filament networks in addition to its membrane remodeling activities.

Dynamin, particularly the ubiquitously expressed dynamin2, was found associated with several actin filament networks, including the F-actin networks of protrusive structures, such as lamellipodia, dorsal ruffles and phagocytic cups, the actin networks of podosomes and invadopodia, and Listeria comet tails and with the actomyosin "belts" at the apical surface of epithelial cells (Chua et al., 2009; Gold et al., 1999; Gray et al., 2005; Krueger et al., 2003; Kurklinsky et al., 2011; Lee and De Camilli, 2002; Orth et al., 2002). One feature shared by most of these actin networks is that they exist as dendritic networks of branched actin filaments formed through the action of the actin nucleator, Arp2/3 complex. Many dynamin interacting partners link dynamin to nucleation promoting factors for Arp2/3 complex, such as N-WASP (Salazar et al., 2003; Schmitz et al., 2004; Soulet et al., 2005), WAVE (Cestra et al., 2005) and cortactin (McNiven et al., 2000; Weed et al., 2000). Thus, dynamin2 associates primarily with dendritic actin filament networks that typically undergo rapid turnover.

\subsubsection{Podosomes and invadopodia.}


Podosomes are dynamic cell-extracellular matrix (ECM) adhesive structures formed at the ventral surface of macrophages, osteoclasts and in Rous sarcoma virusinfected cells (Buccione et al., 2004; Ochoa et al., 2000). Actin filaments of podosomes are highly dynamic and arranged in a radial array surrounding a core of bundled filaments (Luxenburg et al., 2007). ECM degradation occurs at the ventral cell surface in close association with podosomes. Invadopodia are longer-lived than podosomes, possess robust ECM degradation activity and are typically elaborated by invasive tumor cells to enhance metastatic migration of tumor cells through tissues (Artym et al., 2011; Baldassarre et al., 2003; Linder, 2009; Linder et al., 2011).

Dynamin2 was implicated in regulating actin dynamics and/or ECM degrading activity at podosomes and invadopodia via a mechanism that depends on GTP binding and hydrolysis (Baldassarre et al., 2003; Bruzzaniti et al., 2005; Ochoa et al., 2000). Whereas podosomes formed in cells expressing the dominant mutant protein, dynamin2K44A, F-actin turnover at podosomes was decreased, suggesting that dynamin2 GTPase activity regulated F-actin turnover at podosomes (Figure 1) (Ochoa et al., 2000). Exogenous dynamin2-K44A could act in a dominant manner to stabilize filaments by crosslinking (Gu et al., 2010. On the other hand, podosomes and invadopodia failed to form in cells expressing a truncated mutant form of dynamin2 lacking the C-terminal PRD, suggesting a role for full-length dynamin2 in formation or stabilization of these structures \{Lee, 2002 \#512; Mooren et al., 2009; Oser et al., 2009). In osteoclasts, a complex of dynamin2 and the adaptor protein, $\mathrm{Cbl}$, which binds the dynamin2 PRD, may coordinate recruitment of F-actin regulators or activators at podosomes to control osteoclast migration on bone. Phosphorylation by c-Src kinase destabilized the 
dynamin2-Cbl complex, suggesting a link between adhesion signaling, migration and dynamin2-mediated regulation of actin dynamics at podosomes (Bruzzaniti et al., 2005).

Dynamin2 may stabilize F-actin networks of invadopodia in concert with its interacting partner, cortactin, which also binds F-actin (Oser et al., 2009); however, the actions of dynamin2 and cortactin for efficient vesicle trafficking or secretion of ECMdegrading metalloproteases has not been ruled out. In this regard, dynamin2 and cortactin were implicated in formation of secretory vesicles at the trans-Golgi (Cao et al., 2005; Salvarezza et al., 2009; Weller et al., 2010), and cortactin promoted secretion of metalloproteases and ECM degradation at invadopodia (Clark and Weaver, 2008).

\subsubsection{F-actin comets and pedestals}

Many bacterial pathogens of mammalian cells commandeer host cell actin filaments and their regulatory machinery to promote pathogenicity (Haglund and Welch, 2011). Dynamin2 is associated with F-actin structures elaborated by two such bacterial pathogens, the F-actin tails of Listeria monocytogenes and the F-actin pedestals elaborated by enteropathogenic E. coli (Figure 1). Actin tails induced by Listeria propel the bacteria through the cytoplasm, eventually guiding them into protrusions that become engulfed by adjacent neighbor cells. Using this mechanism, Listeria spreads from cellto-cell, evading immune detection. Since motility of Listeria via actin tails does not involve vesicle budding or endocytosis, the presence of dynamin2 along the F-actin tails provided compelling evidence for an actin-dependent and endocytosis-independent function for dynamin2 (Henmi et al., 2011; Lee and De Camilli, 2002; Orth et al., 2002). Although dynamin2 was not required for Listeria motility in reconstituted systems 
(Loisel et al., 1999), dynamin2 may organize actin filaments of comet tails for optimal bacterial movement in the cellular milieu or efficient cell-to-cell spread of Listeria via Factin-rich projections that drill into neighboring cells. The PRD was critical for targeting dynamin2 to F-actin tails (Lee and De Camilli, 2002; Orth et al., 2002). Experimentally induced F-actin tails generated by expression of phosphatidylinositol-4-phosphate, 5kinase (PIP5K) were similarly decorated by dynamin (Orth et al., 2002). Whereas Factin tails formed in Listeria-infected cells depleted of dynamin2, the tails were shorter and Listeria moved more slowly compared to bacteria in infected control cells (Henmi et al., 2011). Expression of dynamin mutant proteins defective in GTPase activity also decreased F-actin tail number, tail length and rate of movement of Listeria and PI5Kinduced comet tails (Henmi et al., 2011; Lee and De Camilli, 2002; Orth et al., 2002). Exogenously expressed mutant dynamin proteins might indirectly influence motility of PIP5K-induced vesicular compartments if, as a result of decreased membrane scission activity in cells expressing mutant dynamins, PI5K-generated vesicles formed less frequently or were larger in size, creating different templates for F-actin tail formation (Cameron et al., 2004).

An intriguing link between dynamin2, Listeria motility and microtubules was recently uncovered. As discussed above, microtubule stability, the content of acetylated tubulin in microtubules and the rate of Listeria motility all depended on the cellular concentration of dynamin2 (Henmi et al., 2011; Tanabe and Takei, 2009). These apparently unrelated findings prompted the suggestion that increased stable microtubules in dynamin2-depleted cells might hinder bacterial movement (Figure 1) (Henmi et al., 2011). In support of this idea, addition of agents that alter the global organization or 
abundance of cellular microtubules restored normal Listeria movement to dynamin2depleted cells. These agents may create a cellular milieu to promote maximal propulsion. In contrast with Listeria invasion of the cytoplasm in infected cells, enteropathogenic E. coli (EPEC) remain bound to the outside of infected cells, anchored at the plasma membrane via an F-actin structure called a pedestal (Goosney et al., 2001). Dynamin2 was implicated in recruiting components of the actin assembly machinery required for F-actin pedestal formation (Figure 1) (Unsworth et al., 2007). EPEC attach to cells via the bacterial protein, Tir, which then recruits the adaptor protein Nck to the intracellular membrane face. Subsequent recruitment of actin polymerizing factors lead to formation of F-actin pedestals (Wunderlich et al., 1999). Depletion of dynamin2 or expression of mutant dynamin proteins decreased the accumulation F-actin at EPEC pedestals, implicating dynamin2 in pedestal formation and/or maintenance. Since dynamin2 appeared at sites of EPEC binding in concert with N-WASP but before Arp2/3 complex, dynamin 2 may create a multi-protein scaffold that recruits factors required for de novo F-actin assembly at pedestals. Alternatively, or in addition, dynamin2 may orchestrate local rearrangements in cortical F-actin at bacterial attachments sites to establish a filament architecture conducive to pedestal formation.

\subsubsection{Dynamin and other dendritic actin networks: lamellipodia, cortical ruffles and}

\section{phagocytic cups}

Dynamin was found associated with dendritic actin networks of lamellipodia (McNiven et al., 2000), of transient cortical ruffles elaborated on the dorsal surface of PDGF-stimulated and unstimulated cells (Krueger et al., 2003; Schafer et al., 2002), and 
of the protrusions extended by macrophages during phagocytosis (Gold et al., 1999). Recruitment of dynamin to some of these actin networks depended on the PRD, most likely via interactions with the PRD- and F-actin interacting protein, cortactin, which is also enriched in these networks (Gray et al., 2005; Krueger et al., 2003; McNiven et al., 2000; Mooren et al., 2009). Expression of mutant dynamin proteins lacking the PRD or having defective GTPase activity, or microinjection of anti-dynamin antibodies, perturbed dynamics of many of these networks (Figure 1). For example, in macrophagelike cells in which dynamin function was perturbed with injected antibodies or by expressing dynamin2-K44A, protrusions still extended around incoming IgG-coated particles, but arrested before engulfment was complete (Di et al., 2003; Gold et al., 1999; Tse et al., 2003). Thus, dynamin2 may remodel actin filament networks within phagocytic cups to complete phagosome closure. However, a role for dynamin2 in modulating exocytic vesicles and their fusion at nascent phagosomes has not been ruled out (Di et al., 2003).

\subsubsection{Dynamin and actomyosin networks}

The participation of dynamin 2 in regulating contractile actomyosin networks was recently uncovered (Figure 1) (Chua et al., 2009; Mooren et al., 2009). Contractile actomyosin networks at the apical junctions maintain the organization of epithelial sheets and drive tissue morphogenesis that shapes developing tissues and organisms (Hartsock and Nelson, 2008; Lecuit and Wieschaus, 2002). Actomyosin networks also comprise a key component of the cell migration machinery (Vicente-Manzanares et al., 2009). Expressed mutant dynamin2 proteins that decrease GTP hydrolysis induced constriction 
at apical junctions of epithelial cells; importantly, perturbing endocytic activity via alternate mechanisms did not induce apical constriction (Chua et al., 2009). Thus, the effects of dynamin2-K44A on contractility did not result solely from decreased endocytosis. Apical constriction by dynamin2-K44A depended on Rho-ROCK signaling and the dynamin2-interacting protein, cortactin. Additionally, depleting dynamin2 in epithelial cells disrupted cell-cell adherens junctions (Chua et al., 2009). Loss of adherens junction integrity in dynamin depleted epithelial cells may result from altered recycling of E-cadherin, which initiates and maintains dynamic junctions (Ivanov et al., 2005). Dynamin2 may have dual roles at adherens junctions: recycling cell-cell adhesion molecules and modulating contractility via pathways that influence actomyosin function. Although dynamin2 was not associated with actomyosin networks in non-epithelial cells, it might influence contractility through effects on the dendritic actin networks that form and maintain actomyosin networks (Ehrlich et al., 2002; Kovacs et al., 2002; Verma et al., 2004). Consistent with this idea, the organization of select actomyosin networks comprising transverse arcs was perturbed in dynamin2-depleted U2-OS cells (Figure 1) (Mooren et al., 2009).

\subsubsection{Neurites, growth cones and dentritic spines}

Paralysis of the Drosophila mutant shibire ${ }^{t s l}$ led investigators to ultimately establish a role for dynamin in recycling synaptic membrane that controls synaptic transmission (van der Bliek and Meyerowitz, 1991). However, cultured neurons from shibire $^{t s l}$ flies also exhibited defects in neurite extension, suggesting that dynamin also influenced actin-dependent processes (Kim and Wu, 1987; Torre et al., 1994). At that 
time, little was known about the mechanisms by which neuronal growth cones migrated to extend neurites, aside from general participation of actin filaments and microtubules (Letourneau et al., 1986; Marsh and Letourneau, 1984). Renewed interest in investigating a role for dynamin in regulating actin-dependent processes in neurons came with the identification of alternatively-spliced isoforms of dynamin3, a dynamin family member that, with dynamin1, was enriched in neuronal tissue. Whereas some splicevariants of dynamin3 worked cooperatively with dynamin1 to support synaptic transmission (Raimondi et al., 2011), other dynamin3 isoforms were highly enriched at the tips of dendritic spines of cultured hippocampal neurons (Gray et al., 2005). When expressed in neurons, one splice variant of dynamin3 (dynamin3-baa) altered the morphology of spine-like protrusions along neurites from short, mushroom-shaped structures to elongated filopodia characteristic of immature dendritic spines (Gray et al., 2003; Gray et al., 2005). The ability of dynamin3-baa to influence spine morphology depended on its interaction with cortactin, implicating dynamin3-baa and cortactin in regulating F-actin-based structures in developing neurons (Gray et al., 2005).

A complex of dynamin2 and cortactin was also implicated in regulating another neuronal appendage, the growth cone (Kurklinsky et al., 2011). Growth cones are dynamic structures at the tips of axons that guide elongating axons during neuronal development. Lamellipodia and filopodia, composed of branched actin filaments and actin filament bundles, respectively, comprise the growth cone periphery. These F-actin networks, together with microtubules in the central body of growth cones, respond to guidance cues and orchestrate the direction and rate of growth cone advance. Cortactin and dynamin2, but not dynamin1 or dynamin3, were localized with adhesion proteins at 
the transition zone between the peripheral and central regions of growth cones. When overexpressed, cortactin or dynamin2 enhanced growth cone spreading and the recruitment of the F-actin crosslinking protein, $\alpha$-actinin, to the transition zone (Kurklinsky et al., 2011). Thus, dynamin2 and cortactin appear to influence the architecture of F-actin networks of growth cones, which in turn, may regulate adhesion to the ECM and the rate of neurite outgrowth.

\subsubsection{Dynamin action during whole cell migration and tumor cell invasion.}

Cell migration requires the concerted actions of many cellular processes: actin filament and microtubule dynamics, formation of adhesive structures, integrin receptor cycling, contractility, and controlled directionality. Dynamin may contribute to cell migration via several non-mutually-exclusive mechanisms: by recycling integrin receptors to influence cell-substrate adhesion (Chao and Kunz, 2009; Ezratty et al., 2005), by regulating secretion of degradative enzymes involved in ECM invasion (Cao et al., 2005), by recruiting actin assembly factors through its PRD-interacting partners to drive protrusive behavior (Kruchten and McNiven, 2006), by generating signaling molecules associated with internalized receptors that regulate the cytoskeleton downstream of endocytic activity (Feng et al., 2012), or by maintaining microtubule organization and dynamics required to maintain cell polarity and focal adhesion disassembly (Ezratty et al., 2005; Kaverina et al., 1999; Tanabe and Takei, 2009; Thompson et al., 2004). As discussed above, dynamin2 is a component of lamellipodia and of growth factor-induced circular ruffles on the plasma membrane, both of which are associated with advancing the leading edge of migrating cells (Krueger et al., 2003; 
McNiven et al., 2000; Schafer et al., 2002). Through dynamin's direct interactions with signaling enzymes, such as the p85 subunit of phosphatidylinositol 3-phosphate kinase (PI3K) (Feng et al., 2012), Src (Cao et al., 2010; Foster-Barber and Bishop, 1998), or FAK (Ezratty et al., 2005; Wang et al., 2011), dynamin could organize signaling complexes that fine-tune the migration machinery for efficient motility. A future challenge is to elucidate the molecular mechanisms by which the dynamin regulates these many different elements that, together, move a cell.

The contributions of dynamin to metastatic tumor progression in cancer may be tumor-specific. Dynamin2 expression was elevated in tissues of patients with aggressive, metastatic pancreatic cancer (Eppinga et al., 2012). In cultured pancreatic cancer cells, cellular levels of dynamin2 correlated directly with whole cell migration rate, ECM invasion and metastatic potential in vivo. The ability of dynamin 2 to promote migration of pancreatic cancer cells depended on phosphorylation by c-Src kinase, which together with interactions of dynamin and FAK, may contribute to focal adhesion disassembly and migration (Ahn et al., 2002; Cao et al., 2010; Ezratty et al., 2005; Wang et al., 2011; Weller et al., 2010). Cellular levels of dynamin 2 similarly correlated directly with cell growth and invasion in an experimental model of glioblastoma, however, in this system, interactions of dynamin2 with PI3K and the phosphatase, SHP-2, were involved (Feng et al., 2012). Thus, in two different model systems, dynamin2 supported metastatic behavior via mechanisms that involve complexes with signaling proteins. Whereas the studies cited above point to a pro-migratory function for dynamin 2 in tumor cell invasion, metastatic progression of cervical squamous cell carcinoma and expression of the ECM- 
degrading metalloprotease, MMP-2, were inversely correlated with dynamin2 expression (Lee et al., 2010).

\subsection{Molecular mechanisms of actin filament regulation by dynamin}

Understanding the molecular mechanisms by which dynamin regulates cytoskeletal filaments is an active goal. Initial clues that dynamin might regulate cytoskeletal filaments arose from identifying its direct interactions with microtubules (Shpetner and Vallee, 1989) and actin regulatory proteins (Ferguson and De Camilli, 2012; Kruchten and McNiven, 2006; McNiven et al., 2000; Schafer, 2004). The list of dynamin-interacting proteins continues to expand, with dynamin now recognized as a versatile molecular scaffold that could assemble a myriad of different protein complexes, including actin assembly factors (Krueger et al., 2003; McNiven et al., 2000; Weed et al., 2000), scaffolds and enzymes involved in signal transduction (Cao et al., 2010; Lai et al., 1999; Okamoto et al., 1999; Salazar et al., 2003) and membrane curvature sensing and generating proteins (Anggono and Robinson, 2007; David et al., 1996; Grabs et al., 1997). Most often, interactions with binding partners occur between the dynamin PRD and SH3 domain(s) of the partner protein. Indeed, a mutant form of dynamin lacking the PRD rarely functions in cells. Among dynamin isoforms, the PRD varies most in primary amino acid sequence (Urrutia et al., 1997). The potential for dynamin family members to participate in a complex array of activities as specified by the diverse interactions of its PRD is vast. In this section, we review recent research that provides mechanistic insight for how dynamin regulates actin filaments. 


\subsubsection{Dynamin and cortactin}

The F-actin binding protein cortactin appears to mediate many actin-dependent processes in which dynamin is implicated to participate. Cortactin binds the dynamin PRD and, like dynamin, is enriched in lamellipodia (McNiven et al., 2000; Weed et al., 2000), dorsal ruffles (Krueger et al., 2003), invadopodia (Clark et al., 2007), podosomes (Tehrani et al., 2006), actin comets (Barroso et al., 2006) and at the trans-Golgi network (Cao et al., 2005). Other activities of cortactin, specifically its ability to bind and activate the actin nucleating activity of Arp2/3 complex and to stabilize branched F-actin junctions (Weaver et al., 2001), highlight cortactin as a key bridge between dynamin and dynamic actin filament networks. Although dynamin was recently found to directly bind actin filaments with sub- $\mu \mathrm{M}$ affinity ( $\mathrm{Gu}$ et al., 2010), cortactin greatly potentiates the association of dynamin with actin filaments in vitro (Gray et al., 2005; Mooren et al., 2009). Several different complexes of dynamin and cortactin, together with other actin regulators or signaling molecules, have been identified (Cao et al., 2005; Chua et al., 2009; Gray et al., 2005; Krueger et al., 2003; Kurklinsky et al., 2011; McNiven et al., 2000). Cortactin also stimulated the basal GTPase activity of dynamin2 in vitro, but the biological significance of this activation is unknown (Mooren et al., 2009).

Biochemical experiments performed with purified proteins in vitro have provided intriguing clues of molecular mechanisms by which dynamin might influence actin filament dynamics and organization. Curiously, dynamin influences actin filaments in vitro via several mechanisms. An ongoing challenge will be to validate these mechanisms in living cells and identify how each may be executed in space and time to influence actin-dependent processes. 
It is likely that dynamin exploits many of the intrinsic activities required for membrane remodeling and scission in its actions on actin filaments. Dynamin2 and cortactin, which together potentiate multivalent actin filament crosslinking, tightly bundled actin filaments in vitro; importantly, GTP hydrolysis by dynamin2 remodeled the bundled filaments from tight to loosely-associated (Mooren et al., 2009). When viewed in real time using total internal reflection fluorescence microscopy, filaments in bundles actively unraveled upon addition of GTP (Mooren et al., 2009). GTP hydrolysis by dynamin 2 also influenced the organization of actin filaments associated with PI-4,5$\mathrm{P}_{2}$-containing liposomes (Schafer et al., 2002). GTPase-dependent changes in the relative orientations of filaments within bundled networks could influence access and binding to filaments of other actin regulatory factors, such as cofilin (Mooren et al., 2009). Moreover, actin filament severing was observed as a result of dynamin2-mediated GTP hydrolysis (Mooren et al., 2009); filament severing could influence filament dynamics either by promoting F-actin turnover or by promoting de novo actin assembly, depending on the cellular context. Thus, GTP hydrolysis by dynamin2 and cortactin may influence actin filaments via a broad range of mechanisms, including promoting actin assembly, promoting actin filament turnover or remodeling filament architecture.

\subsubsection{Direct binding of actin filaments by dynamin}

The recent discovery that dynamin directly binds actin filaments brought a novel twist to the scope of mechanisms by which dynamin might regulate actin filaments. Dynamin bundled actin filaments in vitro via interactions of filaments with conserved basic residues within an $\alpha$-helix of the dynamin stalk (Gu et al., 2010). Interestingly, 
ring-shaped dynamin oligomers preferentially associated with short actin filaments, which also maximally stimulated GTP hydrolysis by dynamin. Unexpectedly, dynamin rings promoted "uncapping" of filaments capped at their barbed end by gelsolin (Gu et al., 2010). GTP hydrolysis was not required to uncap filaments, which occurred in the presence of the non-hydrolyzable GTP analog, GTP $\gamma \mathrm{S}$ (Gu et al., 2010). Like filament severing, dynamin-mediated filament uncapping could either promote actin assembly or promote filament disassembly, depending on the local conditions. Support for the hypothesis that dynamin-mediated filament uncapping promoted actin assembly came from studies of cultured podocytes depleted of dynamin2, in which actomyosincontaining stress fibers were disrupted and the accessibility of actin filament barbed ends was reduced (Figure 1) (Gu et al., 2010).

In summary, a growing body of evidence supports the hypothesis that proteins of the dynamin family act directly, or indirectly via PRD-binding partners, on actin filaments. The GTP hydrolysis-dependent activities of dynamin on actin filaments identified in in vitro experiments could modulate cellular actin networks via several different mechanisms, including stimulating local F-actin assembly, promoting F-actin turnover and remodeling actin filament architecture. Additionally, dynamin may modulate actin networks by acting as a dynamic molecular scaffold that recruits factors for de novo actin assembly (Cestra et al., 2005; Feng et al., 2012; Gomez et al., 2005; Salazar et al., 2003; Soulet et al., 2005; Wang et al., 2011; Xin et al., 2009).

\section{Dynamin mutations, the cytoskeleton and human disease}


Mutations in the gene encoding dynamin2 have been linked to two diseases that affect muscle and peripheral nerve function: centronuclear myopathy (CNM), a disorder resulting in muscle degeneration (Bohm et al., 2012; Durieux et al., 2010), and CharcotMarie Tooth disease (CMT), a neuropathy of peripheral nerves (Zuchner et al., 2005). Clustered mutations in the dynamin 2 stalk and PH domain, along with a few others, have been linked to CNM and CMT (Bitoun et al., 2005; Zuchner et al., 2005). Some diseaseassociated mutant dynamin proteins are associated with microtubules. For example, exogenously expressed dynamin2- $\Delta 3$, a CMT-related dynamin with a small deletion within the PH domain, prominently decorated microtubules and induced increased levels of acetylated tubulin along microtubules of cultured COS-1 cells (Tanabe and Takei, 2009). In other studies, however, cellular levels of acetylated tubulin appeared normal when dynamin2- $\Delta 3$ was expressed at near endogenous levels in fibroblastoid cells lacking wild-type dynamin2 (Liu et al., 2011a). Surprisingly, CME was not perturbed in fibroblastoid cells expressing some common mutant dynamin2 proteins associated with CNM or CMT; rather, slight defects in selected secretory pathways and in non-clathrindependent endocytic pathways were noted (Liu et al., 2011a). To the extent that diseaseassociated mutant dynamin2 proteins have been studied, none perturbed PDGFstimulated macropinocytosis, an actin-dependent endocytic process (Liu et al., 2011a). A recently developed mouse model of $\mathrm{CNM}$ in which a common human mutation, dynamin2-R465W, was expressed in skeletal muscle, highlights a role for dynamin2 in several aspects of muscle tissue homeostasis, including sarcomere assembly, contractility and myofiber integrity. 


\section{Concluding Remarks}

The spectrum of cellular activities in which dynamin is implicated to participate is expanding rapidly. Evidence collected over the past decade bolsters hypotheses that dynamin may broadly influence cytoskeletal actin and microtubule networks via a diverse repertoire of molecular mechanisms. Future work will focus on clarifying the relationship between dynamin's actions during endocytosis or other membrane remodeling events and its actions on cytoskeletal filaments. During endocytosis and exocytosis, dynamin may execute membrane remodeling in concert with actin filament remodeling to maximize efficiency. New mutant dynamin proteins that allow investigators to separate dynamin's activities on membranes and cytoskeletal filaments will be needed to clarify this relationship. Moreover, because the scope of cellular processes in which dynamin modulates the cytoskeleton is broad, it will be necessary to investigate the physiological significance of its actions in different cellular contexts.

Clearly, the focus of most investigations to date has centered on the actin-related functions of dynamin. In this regard, biochemical experiments have offered intriguing clues of potential molecular mechanisms that must now be examined in living cells. Despite its discovery as a microtubule bundling protein, very little is known about the molecular mechanisms by which dynamin influences microtubules. This aspect of dynamin action is particularly mysterious because dynamin does not associate prominently with microtubules in interphase cells yet depleting dynamin impacts microtubule dynamics globally. Identifying how dynamin influences microtubule dynamic instability remains an important focus for future investigations. With respect to actin filaments, GTP hydrolysis by dynamin, in combination with its myriad of direct and 
indirect interactions with actin filaments and their regulators, provide a diverse array of mechanisms by which dynamin could modulate actin filament networks. In keeping with the restricted distribution of dynamin with most networks, dynamin's actions are likely to be in local, rather than global. Site-specific actions of dynamin may serve to coordinate local reorganization of the actin cytoskeleton with respect to other cellular structures, such as at clathrin-coated pits, EPEC pedestals, actin comets and podosomes. However, local actin filament remodeling by dynamin within lamellipodia or adherens junctions may act to ultimately influence actin networks required for contractility and whole cell migration. 


\section{Figure. 1: The various ways by which dynamin influences cytoskeletal networks}

Dynamin influences cytoskeletal networks via a variety of mechanisms, including recruiting actin assembly factors, bundling and cross-linking actin filaments, remodeling actin filaments, promoting actin filament turnover and influencing microtubule dynamics and centrosome cohesion. During endocytosis, dynamin modulates actin filament turnover as clathrin-coated structures separate from the plasma membrane (Ferguson et al., 2009). Dynamin contributes to phagosome formation for phagocytosis (Di et al., 2003; Gold et al., 1999) and influences microtubule dynamics and centrosome cohesion via unknown mechanisms (Tanabe and Takei, 2009; Thompson et al., 2004). Dynamin is involved in formation and stability of podosomes and invadopodia, actin-rich structures that promote migration and ECM degradation in some cells (Buccione et al., 2004; Ochoa et al., 2000; Oser et al., 2009). Dynamin is enriched on the F-actin comet tails elaborated by intracellular bacterial pathogens (Lee and De Camilli, 2002; Orth et al., 2002); the function of dynamin for bacterial motility is not known, but indirect effects arising from the influence of dynamin on microtubule stability are not ruled out (Henmi et al., 2011). F-actin assembly at pedestal structures elaborated by pathogenic E. coli depends on dynamin recruitment to bacterial attachment sites (Unsworth et al., 2007). As a component of the dendritic actin networks of lamellipodia and ruffles, dynamin remodels actin filaments to support filament turnover and actomyosin formation (Chua et al., 2009; Krueger et al., 2003; McNiven et al., 2000; Mooren et al., 2009). Actin filament uncapping by dynamin is implicated in maintaining the actomyosin-containing stress fibers in podocytes (Gu et al., 2010). 


\section{Figure. 1: The various ways by which dynamin influences cytoskeletal networks}

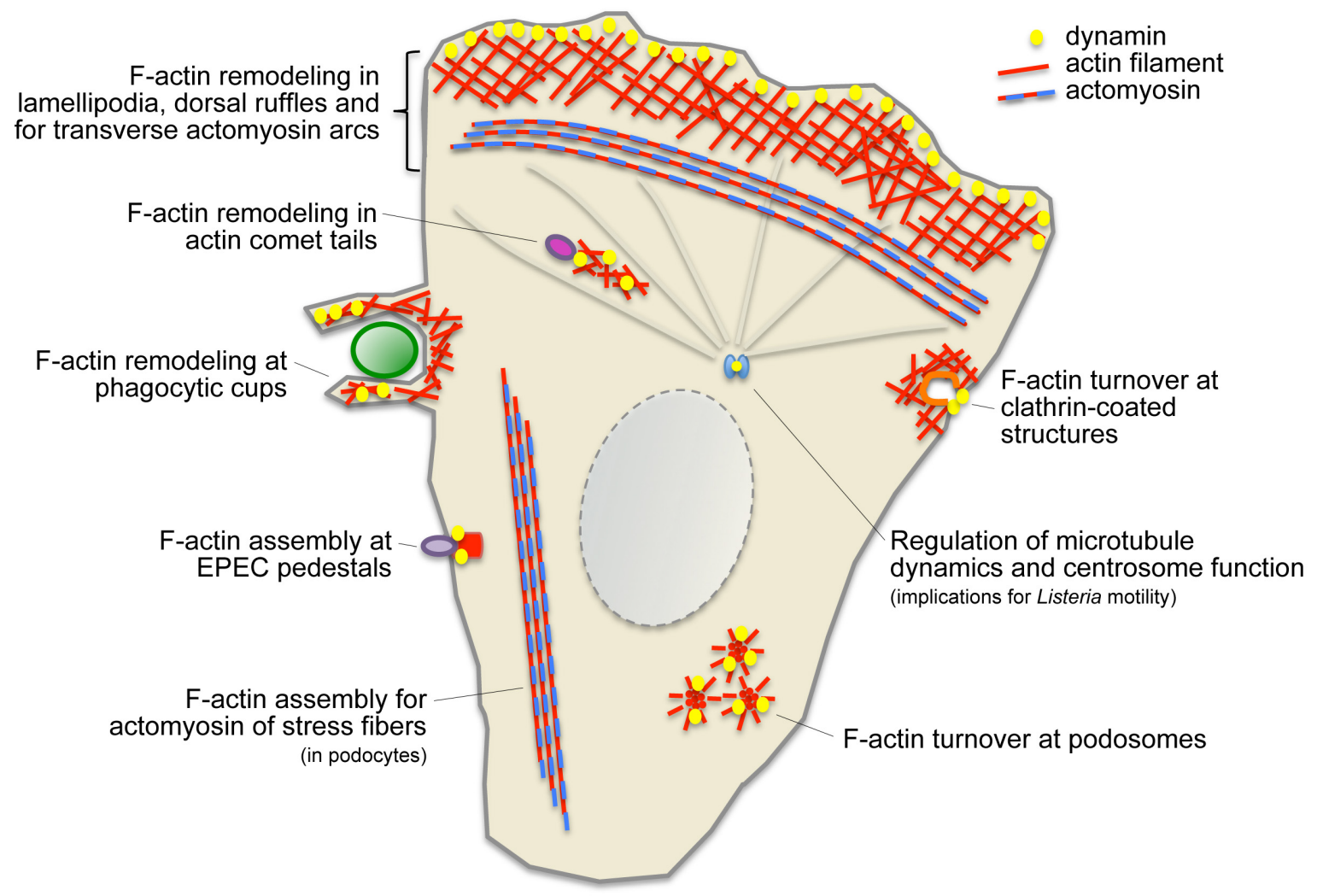




\section{References}

Aghamohammadzadeh, S., and Ayscough, K.R. (2009). Differential requirements for actin during yeast and mammalian endocytosis. Nat Cell Biol 11, 1039-1042.

Ahn, S., Kim, J., Lucaveche, C.L., Reedy, M.C., Luttrell, L.M., Lefkowitz, R.J., and Daaka, Y. (2002). Src-dependent tyrosine phosphorylation regulates dynamin selfassembly and ligand-induced endocytosis of the epidermal growth factor receptor. J Biol Chem 277, 26642-26651.

Anantharam, A., Bittner, M.A., Aikman, R.L., Stuenkel, E.L., Schmid, S.L., Axelrod, D., and Holz, R.W. (2011). A new role for the dynamin GTPase in the regulation of fusion pore expansion. Mol Biol Cell 22, 1907-1918.

Anggono, V., and Robinson, P.J. (2007). Syndapin I and endophilin I bind overlapping proline-rich regions of dynamin I: role in synaptic vesicle endocytosis. J Neurochem 102, 931-943.

Artym, V.V., Matsumoto, K., Mueller, S.C., and Yamada, K.M. (2011). Dynamic membrane remodeling at invadopodia differentiates invadopodia from podosomes. Eur $\mathrm{J}$ Cell Biol 90, 172-180.

Baldassarre, M., Pompeo, A., Beznoussenko, G., Castaldi, C., Cortellino, S., McNiven, M.A., Luini, A., and Buccione, R. (2003). Dynamin participates in focal extracellular matrix degradation by invasive cells. Mol Biol Cell 14, 1074-1084.

Barroso, C., Rodenbusch, S.E., Welch, M.D., and Drubin, D.G. (2006). A role for cortactin in Listeria monocytogenes invasion of NIH $3 \mathrm{~T} 3$ cells, but not in its intracellular motility. Cell Motil Cytoskeleton 63, 231-243.

Barylko, B., Binns, D., Lin, K.M., Atkinson, M.A., Jameson, D.M., Yin, H.L., and Albanesi, J.P. (1998). Synergistic activation of dynamin GTPase by Grb2 and phosphoinositides. J Biol Chem 273, 3791-3797.

Bashkirov, P.V., Akimov, S.A., Evseev, A.I., Schmid, S.L., Zimmerberg, J., and Frolov, V.A. (2008). GTPase cycle of dynamin is coupled to membrane squeeze and release, leading to spontaneous fission. Cell 135, 1276-1286.

Bethoney, K.A., King, M.C., Hinshaw, J.E., Ostap, E.M., and Lemmon, M.A. (2009). A possible effector role for the pleckstrin homology $(\mathrm{PH})$ domain of dynamin. Proc Natl Acad Sci U S A 106, 13359-13364. 
Bitoun, M., Maugenre, S., Jeannet, P.Y., Lacene, E., Ferrer, X., Laforet, P., Martin, J.J., Laporte, J., Lochmuller, H., Beggs, A.H., et al. (2005). Mutations in dynamin 2 cause dominant centronuclear myopathy. Nat Genet 37, 1207-1209.

Bohm, J., Biancalana, V., Dechene, E.T., Bitoun, M., Pierson, C.R., Schaefer, E., Karasoy, H., Dempsey, M.A., Klein, F., Dondaine, N., et al. (2012). Mutation spectrum in the large GTPase dynamin 2, and genotype-phenotype correlation in autosomal dominant centronuclear myopathy. Hum Mutat 33, 949-959.

Bruzzaniti, A., Neff, L., Sanjay, A., Horne, W.C., De Camilli, P., and Baron, R. (2005). Dynamin forms a Src kinase-sensitive complex with $\mathrm{Cbl}$ and regulates podosomes and osteoclast activity. Mol Biol Cell 16, 3301-3313.

Buccione, R., Orth, J.D., and McNiven, M.A. (2004). Foot and mouth: podosomes, invadopodia and circular dorsal ruffles. Nat Rev Mol Cell Biol 5, 647-657.

Cameron, L.A., Robbins, J.R., Footer, M.J., and Theriot, J.A. (2004). Biophysical parameters influence actin-based movement, trajectory, and initiation in a cell-free system. Mol Biol Cell 15, 2312-2323.

Cao, H., Chen, J., Krueger, E.W., and McNiven, M.A. (2010). SRC-mediated phosphorylation of dynamin and cortactin regulates the "constitutive" endocytosis of transferrin. Mol Cell Biol 30, 781-792.

Cao, H., Weller, S., Orth, J.D., Chen, J., Huang, B., Chen, J.L., Stamnes, M., and McNiven, M.A. (2005). Actin and Arf1-dependent recruitment of a cortactin-dynamin complex to the Golgi regulates post-Golgi transport. Nat Cell Biol 7, 483-492.

Cestra, G., Kwiatkowski, A., Salazar, M., Gertler, F., and De Camilli, P. (2005). Tuba, a GEF for CDC42, links dynamin to actin regulatory proteins. Methods Enzymol 404, 537545.

Chao, W.T., and Kunz, J. (2009). Focal adhesion disassembly requires clathrin-dependent endocytosis of integrins. FEBS Lett 583, 1337-1343.

Chappie, J.S., Acharya, S., Leonard, M., Schmid, S.L., and Dyda, F. (2010). G domain dimerization controls dynamin's assembly-stimulated GTPase activity. Nature 465, 435440 . 
Chappie, J.S., Acharya, S., Liu, Y.W., Leonard, M., Pucadyil, T.J., and Schmid, S.L. (2009). An intramolecular signaling element that modulates dynamin function in vitro and in vivo. Mol Biol Cell 20,3561-3571.

Chappie, J.S., Mears, J.A., Fang, S., Leonard, M., Schmid, S.L., Milligan, R.A., Hinshaw, J.E., and Dyda, F. (2011). A pseudoatomic model of the dynamin polymer identifies a hydrolysis-dependent powerstroke. Cell 147, 209-222.

Chen, M.S., Obar, R.A., Schroeder, C.C., Austin, T.W., Poodry, C.A., Wadsworth, S.C., and Vallee, R.B. (1991). Multiple forms of dynamin are encoded by shibire, a Drosophila gene involved in endocytosis. Nature 351, 583-586.

Chircop, M., Sarcevic, B., Larsen, M.R., Malladi, C.S., Chau, N., Zavortink, M., Smith, C.M., Quan, A., Anggono, V., Hains, P.G., et al. (2011). Phosphorylation of dynamin II at serine-764 is associated with cytokinesis. Biochim Biophys Acta 1813, 1689-1699.

Chua, J., Rikhy, R., and Lippincott-Schwartz, J. (2009). Dynamin 2 orchestrates the global actomyosin cytoskeleton for epithelial maintenance and apical constriction. Proc Natl Acad Sci U S A 106, 20770-20775.

Clark, E.S., and Weaver, A.M. (2008). A new role for cortactin in invadopodia: regulation of protease secretion. Eur J Cell Biol 87, 581-590.

Clark, E.S., Whigham, A.S., Yarbrough, W.G., and Weaver, A.M. (2007). Cortactin is an essential regulator of matrix metalloproteinase secretion and extracellular matrix degradation in invadopodia. Cancer Res 67, 4227-4235.

Collins, A., Warrington, A., Taylor, K.A., and Svitkina, T. (2011). Structural organization of the actin cytoskeleton at sites of clathrin-mediated endocytosis. Curr Biol $21,1167-1175$.

Conner, S.D., and Schmid, S.L. (2003). Regulated portals of entry into the cell. Nature $422,37-44$.

Damke, H., Baba, T., Warnock, D.E., and Schmid, S.L. (1994). Induction of mutant dynamin specifically blocks endocytic coated vesicle formation. J Cell Biol 127, 915934.

Danino, D., Moon, K.H., and Hinshaw, J.E. (2004). Rapid constriction of lipid bilayers by the mechanochemical enzyme dynamin. J Struct Biol 147, 259-267. 
David, C., McPherson, P.S., Mundigl, O., and de Camilli, P. (1996). A role of amphiphysin in synaptic vesicle endocytosis suggested by its binding to dynamin in nerve terminals. Proc Natl Acad Sci U S A 93, 331-335.

Di, A., Nelson, D.J., Bindokas, V., Brown, M.E., Libunao, F., and Palfrey, H.C. (2003). Dynamin regulates focal exocytosis in phagocytosing macrophages. Mol Biol Cell 14, 2016-2028.

Doherty, G.J., and McMahon, H.T. (2009). Mechanisms of endocytosis. Annu Rev Biochem 78, 857-902.

Durieux, A.C., Vignaud, A., Prudhon, B., Viou, M.T., Beuvin, M., Vassilopoulos, S., Fraysse, B., Ferry, A., Laine, J., Romero, N.B., et al. (2010). A centronuclear myopathydynamin 2 mutation impairs skeletal muscle structure and function in mice. Hum Mol Genet 19, 4820-4836.

Ehrlich, J.S., Hansen, M.D., and Nelson, W.J. (2002). Spatio-temporal regulation of Rac1 localization and lamellipodia dynamics during epithelial cell-cell adhesion. Dev Cell 3, 259-270.

Eppinga, R.D., Krueger, E.W., Weller, S.G., Zhang, L., Cao, H., and McNiven, M.A. (2012). Increased expression of the large GTPase dynamin 2 potentiates metastatic migration and invasion of pancreatic ductal carcinoma. Oncogene 31, 1228-1241.

Ezratty, E.J., Partridge, M.A., and Gundersen, G.G. (2005). Microtubule-induced focal adhesion disassembly is mediated by dynamin and focal adhesion kinase. Nat Cell Biol 7, 581-590.

Faelber, K., Posor, Y., Gao, S., Held, M., Roske, Y., Schulze, D., Haucke, V., Noe, F., and Daumke, O. (2011). Crystal structure of nucleotide-free dynamin. Nature 477, 556560 .

Feng, H., Liu, K.W., Guo, P., Zhang, P., Cheng, T., McNiven, M.A., Johnson, G.R., Hu, B., and Cheng, S.Y. (2012). Dynamin 2 mediates PDGFRalpha-SHP-2-promoted glioblastoma growth and invasion. Oncogene 31, 2691-2702.

Ferguson, K.M., Lemmon, M.A., Schlessinger, J., and Sigler, P.B. (1994). Crystal structure at 2.2 A resolution of the pleckstrin homology domain from human dynamin. Cell 79, 199-209. 
Ferguson, S.M., and De Camilli, P. (2012). Dynamin, a membrane-remodelling GTPase. Nat Rev Mol Cell Biol 13, 75-88.

Ferguson, S.M., Raimondi, A., Paradise, S., Shen, H., Mesaki, K., Ferguson, A., Destaing, O., Ko, G., Takasaki, J., Cremona, O., et al. (2009). Coordinated actions of actin and BAR proteins upstream of dynamin at endocytic clathrin-coated pits. Dev Cell $17,811-822$.

Ford, M.G., Jenni, S., and Nunnari, J. (2011). The crystal structure of dynamin. Nature $477,561-566$.

Foster-Barber, A., and Bishop, J.M. (1998). Src interacts with dynamin and synapsin in neuronal cells. Proc Natl Acad Sci U S A 95, 4673-4677.

Fulop, T., Doreian, B., and Smith, C. (2008). Dynamin I plays dual roles in the activitydependent shift in exocytic mode in mouse adrenal chromaffin cells. Arch Biochem Biophys 477, 146-154.

Galletta, B.J., Mooren, O.L., and Cooper, J.A. (2010). Actin dynamics and endocytosis in yeast and mammals. Curr Opin Biotechnol 21, 604-610.

Girao, H., Geli, M.I., and Idrissi, F.Z. (2008). Actin in the endocytic pathway: from yeast to mammals. FEBS Lett 582, 2112-2119.

Gold, E.S., Underhill, D.M., Morrissette, N.S., Guo, J., McNiven, M.A., and Aderem, A. (1999). Dynamin 2 is required for phagocytosis in macrophages. J Exp Med 190, 18491856.

Gomez, T.S., Hamann, M.J., McCarney, S., Savoy, D.N., Lubking, C.M., Heldebrant, M.P., Labno, C.M., McKean, D.J., McNiven, M.A., Burkhardt, J.K., et al. (2005). Dynamin 2 regulates $\mathrm{T}$ cell activation by controlling actin polymerization at the immunological synapse. Nat Immunol 6, 261-270.

Gonzalez-Jamett, A.M., Baez-Matus, X., Hevia, M.A., Guerra, M.J., Olivares, M.J., Martinez, A.D., Neely, A., and Cardenas, A.M. (2010). The association of dynamin with synaptophysin regulates quantal size and duration of exocytotic events in chromaffin cells. J Neurosci 30, 10683-10691. 
Goosney, D.L., DeVinney, R., and Finlay, B.B. (2001). Recruitment of cytoskeletal and signaling proteins to enteropathogenic and enterohemorrhagic Escherichia coli pedestals. Infect Immun 69, 3315-3322.

Grabs, D., Slepnev, V.I., Songyang, Z., David, C., Lynch, M., Cantley, L.C., and De Camilli, P. (1997). The SH3 domain of amphiphysin binds the proline-rich domain of dynamin at a single site that defines a new SH3 binding consensus sequence. J Biol Chem 272, 13419-13425.

Gray, N.W., Fourgeaud, L., Huang, B., Chen, J., Cao, H., Oswald, B.J., Hemar, A., and McNiven, M.A. (2003). Dynamin 3 is a component of the postsynapse, where it interacts with mGluR5 and Homer. Curr Biol 13, 510-515.

Gray, N.W., Kruchten, A.E., Chen, J., and McNiven, M.A. (2005). A dynamin-3 spliced variant modulates the actin/cortactin-dependent morphogenesis of dendritic spines. J Cell Sci 118, 1279-1290.

Gu, C., Yaddanapudi, S., Weins, A., Osborn, T., Reiser, J., Pollak, M., Hartwig, J., and Sever, S. (2010). Direct dynamin-actin interactions regulate the actin cytoskeleton. EMBO J 29, 3593-3606.

Haglund, C.M., and Welch, M.D. (2011). Pathogens and polymers: microbe-host interactions illuminate the cytoskeleton. J Cell Biol 195, 7-17.

Hamao, K., Morita, M., and Hosoya, H. (2009). New function of the proline rich domain in dynamin-2 to negatively regulate its interaction with microtubules in mammalian cells. Exp Cell Res 315, 1336-1345.

Hartsock, A., and Nelson, W.J. (2008). Adherens and tight junctions: structure, function and connections to the actin cytoskeleton. Biochim Biophys Acta 1778, 660-669.

Henley, J.R., Krueger, E.W., Oswald, B.J., and McNiven, M.A. (1998). Dynaminmediated internalization of caveolae. J Cell Biol 141, 85-99.

Henmi, Y., Tanabe, K., and Takei, K. (2011). Disruption of microtubule network rescues aberrant actin comets in dynamin2-depleted cells. PLoS One 6, e28603.

Hinshaw, J.E., and Schmid, S.L. (1995). Dynamin self-assembles into rings suggesting a mechanism for coated vesicle budding. Nature 374, 190-192. 
Holroyd, P., Lang, T., Wenzel, D., De Camilli, P., and Jahn, R. (2002). Imaging direct, dynamin-dependent recapture of fusing secretory granules on plasma membrane lawns from PC12 cells. Proc Natl Acad Sci U S A 99, 16806-16811.

Ishida, N., Nakamura, Y., Tanabe, K., Li, S.A., and Takei, K. (2011). Dynamin 2 associates with microtubules at mitosis and regulates cell cycle progression. Cell Struct Funct 36, 145-154.

Itoh, T., Erdmann, K.S., Roux, A., Habermann, B., Werner, H., and De Camilli, P. (2005). Dynamin and the actin cytoskeleton cooperatively regulate plasma membrane invagination by BAR and F-BAR proteins. Dev Cell 9, 791-804.

Ivanov, A.I., Nusrat, A., and Parkos, C.A. (2005). Endocytosis of the apical junctional complex: mechanisms and possible roles in regulation of epithelial barriers. Bioessays 27, 356-365.

Jaiswal, J.K., Rivera, V.M., and Simon, S.M. (2009). Exocytosis of post-Golgi vesicles is regulated by components of the endocytic machinery. Cell 137, 1308-1319.

Jones, S.M., Howell, K.E., Henley, J.R., Cao, H., and McNiven, M.A. (1998). Role of dynamin in the formation of transport vesicles from the trans-Golgi network. Science 279, 573-577.

Kaksonen, M., Peng, H.B., and Rauvala, H. (2000). Association of cortactin with dynamic actin in lamellipodia and on endosomal vesicles. J Cell Sci 113 Pt 24, 44214426.

Kaksonen, M., Sun, Y., and Drubin, D.G. (2003). A pathway for association of receptors, adaptors, and actin during endocytic internalization. Cell 115, 475-487.

Kaksonen, M., Toret, C.P., and Drubin, D.G. (2006). Harnessing actin dynamics for clathrin-mediated endocytosis. Nat Rev Mol Cell Biol 7, 404-414.

Kaverina, I., Krylyshkina, O., and Small, J.V. (1999). Microtubule targeting of substrate contacts promotes their relaxation and dissociation. J Cell Biol 146, 1033-1044.

Kerr, M.C., and Teasdale, R.D. (2009). Defining macropinocytosis. Traffic 10, 364-371. Kessels, M.M., Engqvist-Goldstein, A.E., Drubin, D.G., and Qualmann, B. (2001). Mammalian Abp1, a signal-responsive F-actin-binding protein, links the actin cytoskeleton to endocytosis via the GTPase dynamin. J Cell Biol 153, 351-366. 
Kim, Y.T., and Wu, C.F. (1987). Reversible blockage of neurite development and growth cone formation in neuronal cultures of a temperature-sensitive mutant of Drosophila. J Neurosci 7, 3245-3255.

Klein, D.E., Lee, A., Frank, D.W., Marks, M.S., and Lemmon, M.A. (1998). The pleckstrin homology domains of dynamin isoforms require oligomerization for high affinity phosphoinositide binding. J Biol Chem 273, 27725-27733.

Kovacs, E.M., Goodwin, M., Ali, R.G., Paterson, A.D., and Yap, A.S. (2002). Cadherindirected actin assembly: E-cadherin physically associates with the Arp2/3 complex to direct actin assembly in nascent adhesive contacts. Curr Biol 12, 379-382.

Kruchten, A.E., and McNiven, M.A. (2006). Dynamin as a mover and pincher during cell migration and invasion. J Cell Sci 119, 1683-1690.

Krueger, E.W., Orth, J.D., Cao, H., and McNiven, M.A. (2003). A dynamin-cortactinArp2/3 complex mediates actin reorganization in growth factor-stimulated cells. Mol Biol Cell 14, 1085-1096.

Kurklinsky, S., Chen, J., and McNiven, M.A. (2011). Growth cone morphology and spreading are regulated by a dynamin-cortactin complex at point contacts in hippocampal neurons. J Neurochem 117, 48-60.

Lai, M.M., Hong, J.J., Ruggiero, A.M., Burnett, P.E., Slepnev, V.I., De Camilli, P., and Snyder, S.H. (1999). The calcineurin-dynamin 1 complex as a calcium sensor for synaptic vesicle endocytosis. J Biol Chem 274, 25963-25966.

Lamaze, C., Fujimoto, L.M., Yin, H.L., and Schmid, S.L. (1997). The actin cytoskeleton is required for receptor-mediated endocytosis in mammalian cells. J Biol Chem 272, 20332-20335.

Lanzetti, L. (2007). Actin in membrane trafficking. Curr Opin Cell Biol 19, 453-458.

Lecuit, T., and Wieschaus, E. (2002). Junctions as organizing centers in epithelial cells? A fly perspective. Traffic 3, 92-97.

Lee, E., and De Camilli, P. (2002). Dynamin at actin tails. Proc Natl Acad Sci U S A 99, 161-166.

Lee, Y.Y., Do, I.G., Park, Y.A., Choi, J.J., Song, S.Y., Kim, C.J., Kim, M.K., Song, T.J., Park, H.S., Choi, C.H., et al. (2010). Low dynamin 2 expression is associated with tumor 
invasion and metastasis in invasive squamous cell carcinoma of cervix. Cancer Biol Ther $10,329-335$.

Letourneau, P.C., Shattuck, T.A., and Ressler, A.H. (1986). Branching of sensory and sympathetic neurites in vitro is inhibited by treatment with taxol. J Neurosci 6, 19121917.

Lim, J.P., and Gleeson, P.A. (2011). Macropinocytosis: an endocytic pathway for internalising large gulps. Immunol Cell Biol 89, 836-843.

Linder, S. (2009). Invadosomes at a glance. J Cell Sci 122, 3009-3013.

Linder, S., Wiesner, C., and Himmel, M. (2011). Degrading devices: invadosomes in proteolytic cell invasion. Annu Rev Cell Dev Biol 27, 185-211.

Liu, J., Sun, Y., Oster, G.F., and Drubin, D.G. (2010). Mechanochemical crosstalk during endocytic vesicle formation. Curr Opin Cell Biol 22, 36-43.

Liu, Y.W., Lukiyanchuk, V., and Schmid, S.L. (2011a). Common membrane trafficking defects of disease-associated dynamin 2 mutations. Traffic 12, 1620-1633.

Liu, Y.W., Neumann, S., Ramachandran, R., Ferguson, S.M., Pucadyil, T.J., and Schmid, S.L. (2011b). Differential curvature sensing and generating activities of dynamin isoforms provide opportunities for tissue-specific regulation. Proc Natl Acad Sci U S A 108, E234-242.

Liu, Y.W., Surka, M.C., Schroeter, T., Lukiyanchuk, V., and Schmid, S.L. (2008). Isoform and splice-variant specific functions of dynamin-2 revealed by analysis of conditional knock-out cells. Mol Biol Cell 19, 5347-5359.

Loisel, T.P., Boujemaa, R., Pantaloni, D., and Carlier, M.F. (1999). Reconstitution of actin-based motility of Listeria and Shigella using pure proteins. Nature 401, 613-616.

Luxenburg, C., Geblinger, D., Klein, E., Anderson, K., Hanein, D., Geiger, B., and Addadi, L. (2007). The architecture of the adhesive apparatus of cultured osteoclasts: from podosome formation to sealing zone assembly. PLoS One 2, e179.

Macia, E., Ehrlich, M., Massol, R., Boucrot, E., Brunner, C., and Kirchhausen, T. (2006). Dynasore, a cell-permeable inhibitor of dynamin. Dev Cell 10, 839-850. 
Maeda, K., Nakata, T., Noda, Y., Sato-Yoshitake, R., and Hirokawa, N. (1992). Interaction of dynamin with microtubules: its structure and GTPase activity investigated by using highly purified dynamin. Mol Biol Cell 3, 1181-1194.

Marks, B., Stowell, M.H., Vallis, Y., Mills, I.G., Gibson, A., Hopkins, C.R., and McMahon, H.T. (2001). GTPase activity of dynamin and resulting conformation change are essential for endocytosis. Nature 410, 231-235.

Marsh, L., and Letourneau, P.C. (1984). Growth of neurites without filopodial or lamellipodial activity in the presence of cytochalasin B. J Cell Biol 99, 2041-2047.

McMahon, H.T., and Boucrot, E. (2011). Molecular mechanism and physiological functions of clathrin-mediated endocytosis. Nat Rev Mol Cell Biol 12, 517-533.

McNiven, M.A., Kim, L., Krueger, E.W., Orth, J.D., Cao, H., and Wong, T.W. (2000). Regulated interactions between dynamin and the actin-binding protein cortactin modulate cell shape. J Cell Biol 151, 187-198.

Merrifield, C.J., Feldman, M.E., Wan, L., and Almers, W. (2002). Imaging actin and dynamin recruitment during invagination of single clathrin-coated pits. Nat Cell Biol 4, 691-698.

Merrifield, C.J., Moss, S.E., Ballestrem, C., Imhof, B.A., Giese, G., Wunderlich, I., and Almers, W. (1999). Endocytic vesicles move at the tips of actin tails in cultured mast cells. Nat Cell Biol 1, 72-74.

Merrifield, C.J., Perrais, D., and Zenisek, D. (2005). Coupling between clathrin-coatedpit invagination, cortactin recruitment, and membrane scission observed in live cells. Cell 121, 593-606.

Mooren, O.L., Galletta, B.J., and Cooper, J.A. (2012). Roles for actin assembly in endocytosis. Annu Rev Biochem 81, 661-686.

Mooren, O.L., Kotova, T.I., Moore, A.J., and Schafer, D.A. (2009). Dynamin2 GTPase and cortactin remodel actin filaments. J Biol Chem 284, 23995-24005.

Morita, M., Hamao, K., Izumi, S., Okumura, E., Tanaka, K., Kishimoto, T., and Hosoya, H. (2010). Proline-rich domain in dynamin-2 has a low microtubule-binding activity: how is this activity controlled during mitosis in HeLa cells? J Biochem 148, 533-538. 
Ochoa, G.C., Slepnev, V.I., Neff, L., Ringstad, N., Takei, K., Daniell, L., Kim, W., Cao, H., McNiven, M., Baron, R., et al. (2000). A functional link between dynamin and the actin cytoskeleton at podosomes. J Cell Biol 150, 377-389.

Okamoto, M., Schoch, S., and Sudhof, T.C. (1999). EHSH1/intersectin, a protein that contains $\mathrm{EH}$ and SH3 domains and binds to dynamin and SNAP-25. A protein connection between exocytosis and endocytosis? J Biol Chem 274, 18446-18454.

Orth, J.D., Krueger, E.W., Cao, H., and McNiven, M.A. (2002). The large GTPase dynamin regulates actin comet formation and movement in living cells. Proc Natl Acad Sci U S A 99, 167-172.

Oser, M., Yamaguchi, H., Mader, C.C., Bravo-Cordero, J.J., Arias, M., Chen, X., Desmarais, V., van Rheenen, J., Koleske, A.J., and Condeelis, J. (2009). Cortactin regulates cofilin and N-WASp activities to control the stages of invadopodium assembly and maturation. J Cell Biol 186, 571-587.

Pollard, T.D. (2007). Regulation of actin filament assembly by Arp2/3 complex and formins. Annu Rev Biophys Biomol Struct 36, 451-477.

Poodry, C.A., and Edgar, L. (1979). Reversible alteration in the neuromuscular junctions of Drosophila melanogaster bearing a temperature-sensitive mutation, shibire. J Cell Biol $81,520-527$.

Praefcke, G.J., and McMahon, H.T. (2004). The dynamin superfamily: universal membrane tubulation and fission molecules? Nat Rev Mol Cell Biol 5, 133-147.

Pucadyil, T.J., and Schmid, S.L. (2008). Real-time visualization of dynamin-catalyzed membrane fission and vesicle release. Cell 135, 1263-1275.

Qualmann, B., Kessels, M.M., and Kelly, R.B. (2000). Molecular links between endocytosis and the actin cytoskeleton. J Cell Biol 150, F111-116.

Raimondi, A., Ferguson, S.M., Lou, X., Armbruster, M., Paradise, S., Giovedi, S., Messa, M., Kono, N., Takasaki, J., Cappello, V., et al. (2011). Overlapping role of dynamin isoforms in synaptic vesicle endocytosis. Neuron 70, 1100-1114.

Ramachandran, R. (2011). Vesicle scission: dynamin. Semin Cell Dev Biol 22, 10-17. 
Ramachandran, R., Pucadyil, T.J., Liu, Y.W., Acharya, S., Leonard, M., Lukiyanchuk, V., and Schmid, S.L. (2009). Membrane insertion of the pleckstrin homology domain variable loop 1 is critical for dynamin-catalyzed vesicle scission. Mol Biol Cell 20, 46304639 .

Ramachandran, R., and Schmid, S.L. (2008). Real-time detection reveals that effectors couple dynamin's GTP-dependent conformational changes to the membrane. EMBO J 27, 27-37.

Ringstad, N., Gad, H., Low, P., Di Paolo, G., Brodin, L., Shupliakov, O., and De Camilli, P. (1999). Endophilin/SH3p4 is required for the transition from early to late stages in clathrin-mediated synaptic vesicle endocytosis. Neuron 24, 143-154.

Roux, A., Uyhazi, K., Frost, A., and De Camilli, P. (2006). GTP-dependent twisting of dynamin implicates constriction and tension in membrane fission. Nature 441, 528-531.

Salazar, M.A., Kwiatkowski, A.V., Pellegrini, L., Cestra, G., Butler, M.H., Rossman, K.L., Serna, D.M., Sondek, J., Gertler, F.B., and De Camilli, P. (2003). Tuba, a novel protein containing bin/amphiphysin/Rvs and Dbl homology domains, links dynamin to regulation of the actin cytoskeleton. J Biol Chem 278, 49031-49043.

Salvarezza, S.B., Deborde, S., Schreiner, R., Campagne, F., Kessels, M.M., Qualmann, B., Caceres, A., Kreitzer, G., and Rodriguez-Boulan, E. (2009). LIM kinase 1 and cofilin regulate actin filament population required for dynamin-dependent apical carrier fission from the trans-Golgi network. Mol Biol Cell 20, 438-451.

Sauvonnet, N., Dujeancourt, A., and Dautry-Varsat, A. (2005). Cortactin and dynamin are required for the clathrin-independent endocytosis of gammac cytokine receptor. J Cell Biol 168, 155-163.

Schafer, D.A. (2004). Regulating actin dynamics at membranes: a focus on dynamin. Traffic 5, 463-469.

Schafer, D.A., Weed, S.A., Binns, D., Karginov, A.V., Parsons, J.T., and Cooper, J.A. (2002). Dynamin2 and cortactin regulate actin assembly and filament organization. Curr Biol 12, 1852-1857.

Schlunck, G., Damke, H., Kiosses, W.B., Rusk, N., Symons, M.H., Waterman-Storer, C.M., Schmid, S.L., and Schwartz, M.A. (2004). Modulation of Rac localization and function by dynamin. Mol Biol Cell 15, 256-267. 
Schmid, S.L., and Frolov, V.A. (2011). Dynamin: functional design of a membrane fission catalyst. Annu Rev Cell Dev Biol 27, 79-105.

Schmitz, U., Thommes, K., Beier, I., Dusing, R., and Vetter, H. (2004). Identification of Nck interacting proteins in vascular smooth muscle cells. Clin Exp Hypertens 26, 267275.

Shajahan, A.N., Timblin, B.K., Sandoval, R., Tiruppathi, C., Malik, A.B., and Minshall, R.D. (2004). Role of Src-induced dynamin-2 phosphorylation in caveolae-mediated endocytosis in endothelial cells. J Biol Chem 279, 20392-20400.

Shpetner, H.S., Herskovits, J.S., and Vallee, R.B. (1996). A binding site for SH3 domains targets dynamin to coated pits. J Biol Chem 271, 13-16.

Shpetner, H.S., and Vallee, R.B. (1989). Identification of dynamin, a novel mechanochemical enzyme that mediates interactions between microtubules. Cell 59, 421432.

Shpetner, H.S., and Vallee, R.B. (1992). Dynamin is a GTPase stimulated to high levels of activity by microtubules. Nature 355, 733-735.

Song, B.D., Leonard, M., and Schmid, S.L. (2004a). Dynamin GTPase domain mutants that differentially affect GTP binding, GTP hydrolysis, and clathrin-mediated endocytosis. J Biol Chem 279, 40431-40436.

Song, B.D., Yarar, D., and Schmid, S.L. (2004b). An assembly-incompetent mutant establishes a requirement for dynamin self-assembly in clathrin-mediated endocytosis in vivo. Mol Biol Cell 15, 2243-2252.

Soulet, F., Yarar, D., Leonard, M., and Schmid, S.L. (2005). SNX9 regulates dynamin assembly and is required for efficient clathrin-mediated endocytosis. Mol Biol Cell 16, 2058-2067.

Stowell, M.H., Marks, B., Wigge, P., and McMahon, H.T. (1999). Nucleotide-dependent conformational changes in dynamin: evidence for a mechanochemical molecular spring. Nat Cell Biol 1, 27-32.

Sundborger, A., Soderblom, C., Vorontsova, O., Evergren, E., Hinshaw, J.E., and Shupliakov, O. (2011). An endophilin-dynamin complex promotes budding of clathrincoated vesicles during synaptic vesicle recycling. J Cell Sci 124, 133-143. 
Takei, K., McPherson, P.S., Schmid, S.L., and De Camilli, P. (1995). Tubular membrane invaginations coated by dynamin rings are induced by GTP-gamma $\mathrm{S}$ in nerve terminals. Nature 374, 186-190.

Takei, K., Slepnev, V.I., Haucke, V., and De Camilli, P. (1999). Functional partnership between amphiphysin and dynamin in clathrin-mediated endocytosis. Nat Cell Biol 1, 3339.

Tanabe, K., and Takei, K. (2009). Dynamic instability of microtubules requires dynamin 2 and is impaired in a Charcot-Marie-Tooth mutant. J Cell Biol 185, 939-948.

Taylor, M.J., Lampe, M., and Merrifield, C.J. (2012). A feedback loop between dynamin and actin recruitment during clathrin-mediated endocytosis. PLoS Biol 10, e1001302.

Tehrani, S., Faccio, R., Chandrasekar, I., Ross, F.P., and Cooper, J.A. (2006). Cortactin has an essential and specific role in osteoclast actin assembly. Mol Biol Cell 17, 28822895.

Thompson, H.M., Cao, H., Chen, J., Euteneuer, U., and McNiven, M.A. (2004). Dynamin 2 binds gamma-tubulin and participates in centrosome cohesion. Nat Cell Biol 6, 335342.

Thompson, H.M., Skop, A.R., Euteneuer, U., Meyer, B.J., and McNiven, M.A. (2002). The large GTPase dynamin associates with the spindle midzone and is required for cytokinesis. Curr Biol 12, 2111-2117.

Torre, E., McNiven, M.A., and Urrutia, R. (1994). Dynamin 1 antisense oligonucleotide treatment prevents neurite formation in cultured hippocampal neurons. J Biol Chem 269, 32411-32417.

Tse, S.M., Furuya, W., Gold, E., Schreiber, A.D., Sandvig, K., Inman, R.D., and Grinstein, S. (2003). Differential role of actin, clathrin, and dynamin in Fc gamma receptor-mediated endocytosis and phagocytosis. J Biol Chem 278, 3331-3338.

Unsworth, K.E., Mazurkiewicz, P., Senf, F., Zettl, M., McNiven, M., Way, M., and Holden, D.W. (2007). Dynamin is required for F-actin assembly and pedestal formation by enteropathogenic Escherichia coli (EPEC). Cell Microbiol 9, 438-449. 
Urrutia, R., Henley, J.R., Cook, T., and McNiven, M.A. (1997). The dynamins: redundant or distinct functions for an expanding family of related GTPases? Proc Natl Acad Sci U S A $94,377-384$.

van der Bliek, A.M., and Meyerowitz, E.M. (1991). Dynamin-like protein encoded by the Drosophila shibire gene associated with vesicular traffic. Nature 351, 411-414.

Verma, S., Shewan, A.M., Scott, J.A., Helwani, F.M., den Elzen, N.R., Miki, H., Takenawa, T., and Yap, A.S. (2004). Arp2/3 activity is necessary for efficient formation of E-cadherin adhesive contacts. J Biol Chem 279, 34062-34070.

Vicente-Manzanares, M., Ma, X., Adelstein, R.S., and Horwitz, A.R. (2009). Non-muscle myosin II takes centre stage in cell adhesion and migration. Nat Rev Mol Cell Biol 10, 778-790.

Wang, Y., Cao, H., Chen, J., and McNiven, M.A. (2011). A direct interaction between the large GTPase dynamin-2 and FAK regulates focal adhesion dynamics in response to active Src. Mol Biol Cell 22, 1529-1538.

Warnock, D.E., and Schmid, S.L. (1996). Dynamin GTPase, a force-generating molecular switch. Bioessays 18, 885-893.

Weaver, A.M., Karginov, A.V., Kinley, A.W., Weed, S.A., Li, Y., Parsons, J.T., and Cooper, J.A. (2001). Cortactin promotes and stabilizes Arp2/3-induced actin filament network formation. Curr Biol 11, 370-374.

Weed, S.A., Karginov, A.V., Schafer, D.A., Weaver, A.M., Kinley, A.W., Cooper, J.A., and Parsons, J.T. (2000). Cortactin localization to sites of actin assembly in lamellipodia requires interactions with F-actin and the Arp2/3 complex. J Cell Biol 151, 29-40.

Weinberg, J., and Drubin, D.G. (2012). Clathrin-mediated endocytosis in budding yeast. Trends Cell Biol 22, 1-13.

Weller, S.G., Capitani, M., Cao, H., Micaroni, M., Luini, A., Sallese, M., and McNiven, M.A. (2010). Src kinase regulates the integrity and function of the Golgi apparatus via activation of dynamin 2. Proc Natl Acad Sci U S A 107, 5863-5868.

Wunderlich, L., Farago, A., and Buday, L. (1999). Characterization of interactions of Nck with Sos and dynamin. Cell Signal 11, 25-29. 
Xin, X., Rabiner, C.A., Mains, R.E., and Eipper, B.A. (2009). Kalirin12 interacts with dynamin. BMC Neurosci 10,61.

Yamada, H., Abe, T., Li, S.A., Masuoka, Y., Isoda, M., Watanabe, M., Nasu, Y., Kumon, H., Asai, A., and Takei, K. (2009). Dynasore, a dynamin inhibitor, suppresses lamellipodia formation and cancer cell invasion by destabilizing actin filaments. Biochem Biophys Res Commun 390, 1142-1148.

Zheng, J., Cahill, S.M., Lemmon, M.A., Fushman, D., Schlessinger, J., and Cowburn, D. (1996). Identification of the binding site for acidic phospholipids on the $\mathrm{pH}$ domain of dynamin: implications for stimulation of GTPase activity. J Mol Biol 255, 14-21.

Zhu, J., Zhou, K., Hao, J.J., Liu, J., Smith, N., and Zhan, X. (2005). Regulation of cortactin/dynamin interaction by actin polymerization during the fission of clathrincoated pits. J Cell Sci 118, 807-817.

Zuchner, S., Noureddine, M., Kennerson, M., Verhoeven, K., Claeys, K., De Jonghe, P., Merory, J., Oliveira, S.A., Speer, M.C., Stenger, J.E., et al. (2005). Mutations in the pleckstrin homology domain of dynamin 2 cause dominant intermediate Charcot-MarieTooth disease. Nat Genet 37, 289-294. 


\section{Chapter 3}

Dynamin2 remodels lamellipodial actin networks to orchestrate lamellar actomyosin

This chapter is under preparation for publication 


\section{Summary}

Actin networks in migrating cells exist as several interdependent structures: sheet-like networks of branched actin filaments in lamellipodia; linear arrays of bundled actin filaments co-assembled with myosin II in lamellae; and actin filaments that couple lamellar actomyosin to focal adhesions. These networks are integrated and coordinated to maintain a coherent actin cytoskeleton as cells move. We show that the large GTPase dynamin2 is enriched in the distal lamellipod where it functions to integrate lamellipodial and lamellar actin networks as they form and flow in U2-OS cells. Dynamin2 remodels actin filaments of the distal lamellipod where it influences the spatiotemporal distribution of $\alpha$-actinin. Perturbing dynamin2 activity disrupted formation of lamellar actomyosin arcs that emerge along $\alpha$-actinin-decorated cables oriented parallel to the cell edge. In the presence of dynamin2, arcs couple to focal adhesions, linking the contractile lamellar actin network to adhesions. Actin filament remodeling by dynamin2 required direct and indirect interactions with actin filaments and finely tuned GTP hydrolysis. Expression in dynamin2-depleted cells of mutant dynamin2 proteins that restore endocytic activity, but not actin remodeling activity, showed that actin remodeling by dynamin 2 was independent of its functions in endocytosis. We conclude that dynamin2 acts within lamellipodia to organize actin filaments required for efficient assembly of lamellar actomyosin. We hypothesize that dynamin2 orchestrates the formation within lamellipodia of a structural template for assembly of lamellar actomyosin capable of engaging at focal adhesions. In this way, dynamin2 acts at a distance to influence the global actin cytoskeleton. 


\section{Introduction}

Cells assemble a spectrum of dynamic actin networks comprised of branched, crosslinked and bundled filaments that, together, move cells and organelles, enable cell division and organize cells within tissues. The best-characterized actin networks are the sheet-like, dendritic networks of lamellipodia that support protrusion of cell membranes and the contractile actomyosin networks of lamellae. Much is known about the biochemical mechanisms by which individual actin networks form in migrating cells, but the processes by which different actin networks are integrated in time and space to maintain a dynamic, but coherent, actin cytoskeleton are less well understood.

In migrating cells, the lamellipodial, lamellar and focal adhesion-associated actin networks are interdependent. Adhesions are initiated within active lamellipodia but mature in response to tension generated by lamellar actomyosin (Choi et al., 2008). Lamellar actomyosin forms, in part, from Arp2/3-dependent actin filaments of lamellipodia and from remnants of lamellipodial F-actin-rich structures such as filopodia or F-actin of retracting protrusions (Anderson et al., 2008; Burnette et al., 2011; Heath, 1983; Hotulainen and Lappalainen, 2006; Nemethova et al., 2008; Tojkander et al., 2012). The centripetal flow of lamellar actomyosin arcs is regulated by the strength of the coupling between the lamellar networks and nascent adhesions (Alexandrova et al., 2008; Burnette et al., 2011; Gardel et al., 2008). The actin filament architecture within these different networks is important for both their individual and interdependent physiologic functions.

Coordination of lamellipodial and lamellar actin networks requires architectural linkages between them and with sites of cell adhesion to the ECM. Actin filament 
crosslinks have emerged as an essential architectural motif for coupling lamellipodial and lamellar actin networks to each other and at sites of cell adhesion (Choi et al., 2008; Oakes et al., 2012). Depleting the actin filament crosslinker, $\alpha$-actinin, which is a component of both lamellipodial and lamellar actin networks and at focal adhesions, perturbed formation of nascent adhesions within the lamellipodia. Re-expressing a mutant myosin II heavy chain that crosslinks filaments but does not exert force on them, restored nascent adhesions to $\alpha$-actinin-depleted cells (Choi et al., 2008). Although maturation of adhesions depended on threshold levels of tension generated by myosin II, $\alpha$-actinin-crosslinked filaments that organized and stabilized lamellar actin networks were also required (Oakes et al., 2012). Similarly, $\alpha$-actinin is part of the molecular linkage between the lamellar actomyosin and adhesions that triggers its transition from fast to slow flow (Brown et al., 2006; Hu et al., 2007; Kanchanawong et al., 2010). The strength of this linkage influences force transmission to the extracellular substrate (Gardel et al., 2010; Giannone et al., 2009; Oakes et al., 2012; Schwarz and Gardel, 2012). Although the molecular mechanisms that regulate the interdependent relationships among actin filament networks near the leading edge are complex, we report here that the large GTPase dynamin2 is involved.

Dynamin2 is increasingly acknowledged to regulate elements of both the actin and microtubule cytoskeletons, but its mechanisms of action and the functions executed by dynamin2 on cytoskeletal filaments are unknown (Hamao et al., 2009; Henmi et al., 2011; Kruchten and McNiven, 2006; Menon and Schafer, 2013; Tanabe and Takei, 2009; Thompson et al., 2004). Dynamins bind and bundle actin filaments in vitro (Gu et al., 2010), an activity that is enhanced by proteins such as cortactin that bind dynamin's C- 
terminal proline-rich domain (PRD) (Chua et al., 2009; McNiven et al., 2000; Mooren et al., 2009). In the presence of cortactin, stimulated dynamin2 GTPase activity remodeled bundled filaments in vitro, resulting in filament severing and altering filament packing within the bundles (Mooren et al., 2009). In cultured cells, dynamin2 influenced the actin cytoskeleton in a cell-type and context-specific manner, as determined primarily from dynamin2-depletion experiments. In osteosarcoma cells, dynamin2 was implicated in organizing lamellar actomyosin (Mooren et al., 2009). In epithelial cells, dynamin2 loss perturbed cell-cell junctions and expressed mutant dynamin 2 increased contractility of apical actomyosin (Chua et al., 2009). In podocytes from kidney glomerulus, dynamin2 maintained actin stress fibers that anchored podocytes to the culture substratum $(\mathrm{Gu}$ et al., 2010). In fibroblasts lacking both dynamin 1 and dynamin2, F-actin accumulated at clathrin-coated structures suggesting a role for dynamin2 in promoting actin turnover at these sites (Ferguson et al., 2009). Each of the studies described above was performed on fixed cells, making it difficult to deduce mechanisms by which dynamin2 acts on dynamic actin networks. In this study, we observed lamellipodial and lamellar actin networks in living U2-OS cells subjected to siRNA-mediated downregulation of dynamin2. Our data identify a role for dynamin2 in remodeling lamellipodial actin networks to influence lamellar actomyosin assembly and flow. 


\section{Results}

\section{Dynamin2 influences formation of lamellar actomyosin}

Dynamin2 was implicated in regulating actomyosin networks in osteosarcoma cells, podocytes and epithelial cells (Chua et al., 2009; Mooren et al., 2009; Gu et al., 2010). To determine how dynamin2, which is enriched in lamellipodial actin networks (Krueger et al., 2003; McNiven et al., 2000; Razidlo et al., 2013; Schafer et al., 2002) also influences lamellar actomyosin, we observed formation of nascent actomyosin in living U2-OS cells with and without depletion of dynamin2 using siRNAs. U2-OS cells assemble several actomyosin structures, including transverse arcs aligned parallel to the cell edge (Aratyn-Schaus et al., 2011; Hotulainen and Lappalainen, 2006; Mooren et al., 2009; Oakes et al., 2012; Tojkander et al., 2012). Transverse arcs were proposed to arise, in part, from lamellipodial F-actin and the early steps in their assembly occur near the boundary between the lamellipod and the lamellum (Anderson et al., 2008; Burnette et al., 2011; Hotulainen and Lappalainen, 2006; Nemethova et al., 2008). Once formed, transverse arcs flow retrogradely and interact with dorsal fibers oriented orthogonal to the arcs and anchored via a single focal adhesion near the cell periphery (Burridge and Wittchen, 2013; Hotulainen and Lappalainen, 2006; Naumanen et al., 2008; Oakes et al., 2012).

To observe actomyosin and transverse arcs assemble, we acquired time-lapse movies of cells expressing GFP-myosin light chain 2 (MLC2), a marker for non-muscle myosin II, and mCherry (mCh)- $\alpha$-actinin, a marker for actin filaments (Fig. 1A, Movie 1). Several features of transverse arc formation were apparent in movies of control siRNAtreated cells. Nascent assemblies of non-muscle myosin II appeared near the lamellipod- 
lamellum boundary as small uniformly sized punctae of GFP-MLC2, approximately 0.6 microns in width. GFP-MLC2 punctae appeared at a rate of $\sim 0.8$ punctae $/ \mathrm{min} / 10 \mu \mathrm{m}$ of cell edge and flowed retrogradely (Fig. 1E,F). GFP-MLC2 punctae generally maintained a uniform size and adjacent punctae became aligned in parallel with the cell edge as they moved rearward together. In contrast, the spatiotemporal distribution of mCh- $\alpha$-actinin in lamellipodial and lamellar actin networks was complex. $\mathrm{mCh}-\alpha$-actinin was enriched in lamellipodia where it flowed rearward at a fast rate. $\mathrm{mCh}-\alpha$-actinin also accumulated at focal adhesions and decorated curved, cable-like structures that bridged adhesions as the cables flowed rearward (Fig. 1A, arrowheads and Movie 1) (Rossier et al., 2010). GFP-MLC2 punctae slowed in concert with the cables, suggesting that actomyosin assembled along cables to form the transverse arcs. As transverse arcs matured, the distribution of mCh- $\alpha$-actinin became discontinuous and interspersed with punctae of GFP-MLC2. In contrast with other reports that nascent actomyosin arcs form by coalescence of punctae of MLC2 and $\alpha$-actinin (Hotulainen and Lappalainen, 2006), the earliest actomyosin structures we observed existed as cables decorated uniformly with mCh- $\alpha$-actinin; sub-assemblies of mCh- $\alpha$-actinin-decorated F-actin were not resolved.

To assess the function of dynamin 2 in assembly of transverse arcs, we depleted all isoforms of dynamin2 by nucleofecting siRNAs targeting dynamin2. As in our previous studies (Mooren et al., 2009), 89\% \pm 2.6 s.e.m. of dynamin2 was uniformly depleted in cultured cells using this approach (Fig. 1D). As previously observed in fixed cells (Mooren et al., 2009), transverse arcs formed in dynamin2-depleted cells, but the actomyosin was poorly organized (Fig. 1A,B and Movie 1). Moreover, the dynamic behavior and retrograde flow of nascent transverse arcs in dynamin2-depleted cells 
differed strikingly from those in control siRNA-treated cells (Movie 1). Nascent punctae of GFP-MLC2 appeared more frequently in dyn2-depleted cells (Fig. 1F) and some punctae acquired irregular shapes, either expanding in size or merging with adjacent punctae as they flowed rearward (Movie 1). Transverse arcs flowed retrogradely $~ 1.5-$ fold faster in dyn2-depleted cells compared to arcs in control siRNA-treated cells. Importantly, the rate of retrograde flow was slowed in dyn2-depleted cells to that observed in control cells upon expression of wild type mCh-dynamin2 (Fig. 1E). Unlike the uniformly radial pattern of retrograde flow in control cells, retrograde flow patterns were non-uniform in some dynamin2-depleted cells (Movie 1). The aberrant behavior of GFP-MLC2 punctae in dynamin2-depleted cells was apparent by kymography (Fig. 1B) and in brightest point z-projected images generated from 40 frames of the time-lapse movies (Fig. 1C).

As observed in control cells, mCh- $\alpha$-actinin was diffusely distributed within lamellipodia of dynamin2-depleted cells, but distinct cable-like structures that served as templates of nascent actomyosin were not apparent (Fig. 1A, Movie 1). Cable-like structures that did form in dynamin2-depleted cells appeared discontinuous and transient (Movie 1). Although focal adhesions formed near the lamellipod-lamellar boundary in dynamin2-depleted cells (see below, Fig. 7), mCh- $\alpha$-actinin was not enriched at them, suggesting that dynamin2 influenced the protein composition at adhesions. We conclude that dynamin2 influences the formation of actomyosin arcs and their retrograde flow in U2-OS cells. Because dynamin2 is enriched in lamellipodial F-actin networks (Krueger et al., 2003; McNiven et al., 2000; Razidlo et al., 2013; Schafer et al., 2002) (and see Fig. 
3 below), we hypothesize that dynamin 2 influences actomyosin assembly and dynamics of transverse arcs via its actions on lamellipodial actin filaments.

To rule out that affects of dynamin2 loss on actomyosin result secondarily from alterations in endocytic pathways, we quantified internalization of two membrane receptors. Importantly, the rate of internalization of transferrin receptor and $\beta 1$-integrin, as well as their steady state surface amounts, were similar in control and dynamin2depleted cells (Fig. 2 A, B). Although the lack of an effect on receptor internalization in dynamin2-depleted cells was unexpected, endocytosis could be executed by dynamin 1 (which is expressed in U2-OS cells (Fig. 2C)), by residual dynamin2, or by an unidentified compensatory endocytic pathway. Based on these results, we conclude that effects of depleting dynamin 2 on actin networks and actomyosin do not result from perturbations in receptor endocytosis. Other experiments using mutant dynamin2 proteins that are competent for endocytosis, but do not fully restore transverse arcs (see below), lend additional support to the conclusion that dynamin2 regulates actin filaments independently from its actions in endocytosis. 


\section{Figure 1: Dynamin2 influences actomyosin assembly in U2-OS cells.}

(A) Single-frame images from movies of control and dynamin2-depleted U2-OS cells transiently expressing GFP-MLC2 and mCh- $\alpha$-actinin (see Movie 1). Blue arrowheads identify $\alpha$-actinin-decorated cables in control U2-OS cells that engage focal adhesions during retrograde flow. Scale bar: $2 \mu \mathrm{m}$

(B) Representative kymographs generated from pixel-wide segments perpendicular to the cell edge of movies of control and dyn2-depleted cells expressing GFP-MLC2 and mCh$\alpha$-actinin. (C) Brightest point projected images generated from 40 frames of the GFPMLC2 movie highlight differences in actomyosin retrograde flow patterns in control and dyn2-depleted cells. Scale bar: $2 \mu \mathrm{m}$

(D) Western blot showing dyn 2 in $2 \times 10^{5}$ control and dyn2-depleted cells; actin is the loading control.

(E) Bar graphs indicate the retrograde flow rate of GFP-MLC2 punctae in control, dyn2depleted and in dyn2-depleted cells expressing mCh-WT-dyn2. Error bars indicate the s.e.m; data compiled from two independent experiments. $* * \mathrm{p}<0.005$; ns, not significant.

(F) Bar graphs depicting the number of nascent GFP-MLC2 punctae formed along $10 \mu \mathrm{m}$ of the cell membrane in 20 minutes. Error bars indicate the s.e.m.; data compiled from three independent experiments. ${ }^{*} \mathrm{p}<0.05, * * \mathrm{p}<0.005$. Scale bars: $2 \mu \mathrm{m}$. 
Figure. 1: Dynamin2 influences actomyosin assembly in U2-OS cells.

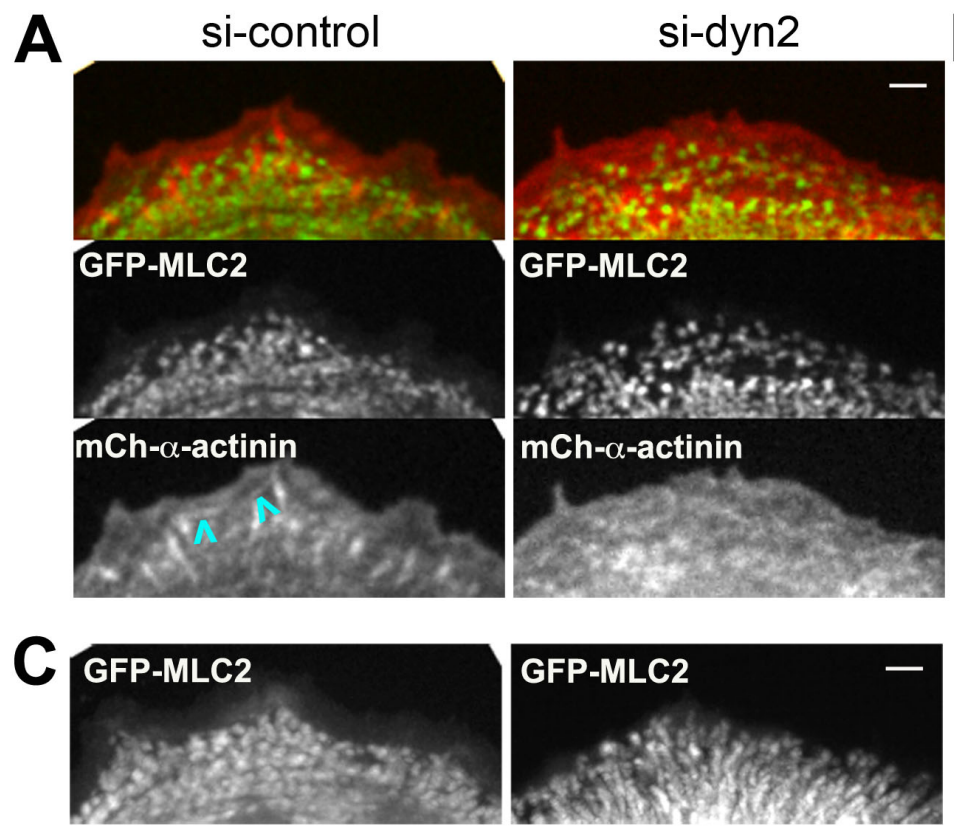

z-projected images
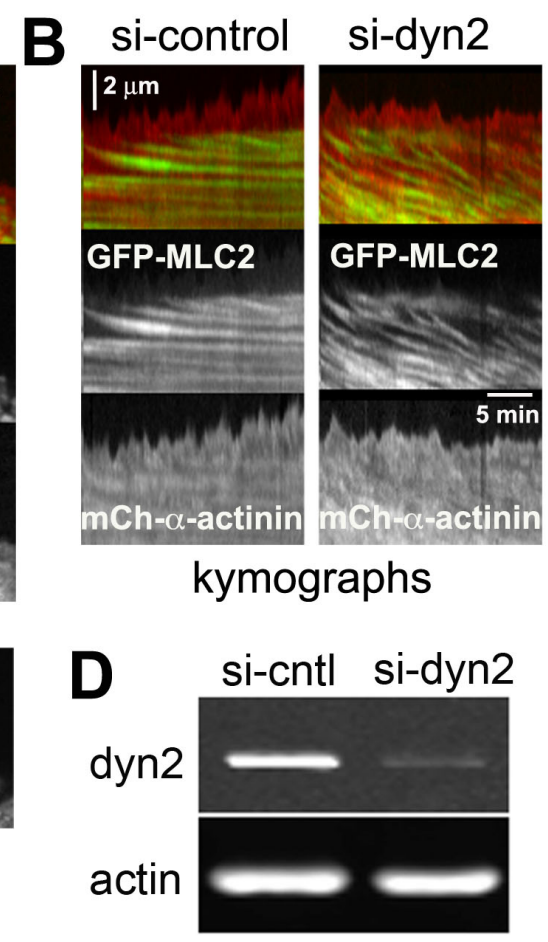

E

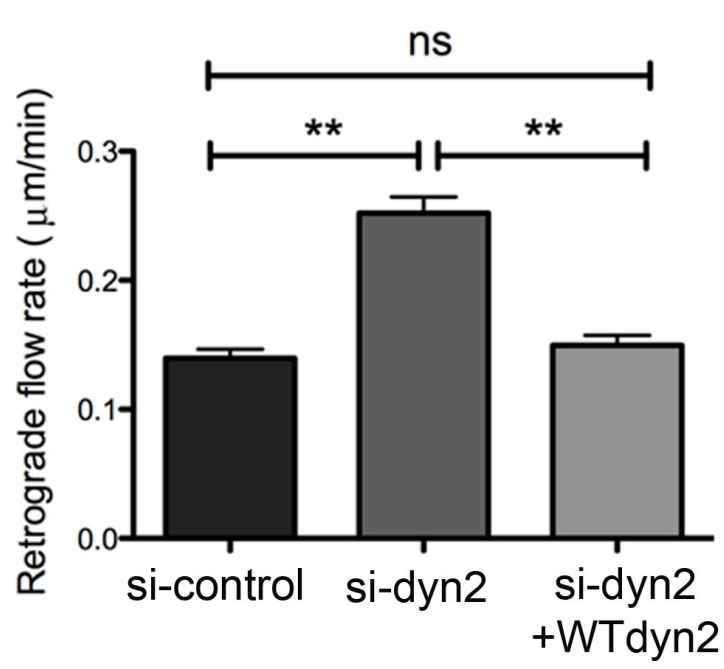

$\mathbf{F}$

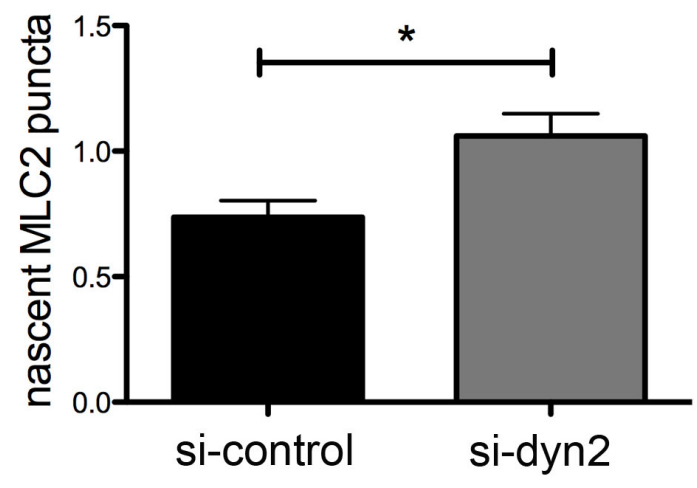


Figure 2: Depleting dynamin2 does not significantly affect the rate of internalization of integrin $\beta 1$ or transferrin receptor.

(A) Plotted is the percentage of integrin $\beta 1$ (top) or transferrin receptor (bottom) internalized by control and dynamin2-depleted cells vs. time after switch to $37^{\circ} \mathrm{C}$.

(B) Total surface level of integrin $\beta 1$ (left) and TfnR (right) for control and dyn2depleted cells. Plotted is the intensity of the fluorophore-tagged primary antibody specific for each receptor for cells at $\mathrm{t}=0 \mathrm{~min}$.

(C) Western blots of whole cell lysates from control and dyn2-depleted cells probed with antibodies to detect dynamin2, integrin $\beta 1, \mathrm{TfnR}$, actin and dynamin 1 , as indicated. 
Figure. 2: Depleting dynamin2 does not significantly affect the rate of internalization of integrin $\beta 1$ or transferrin receptor.
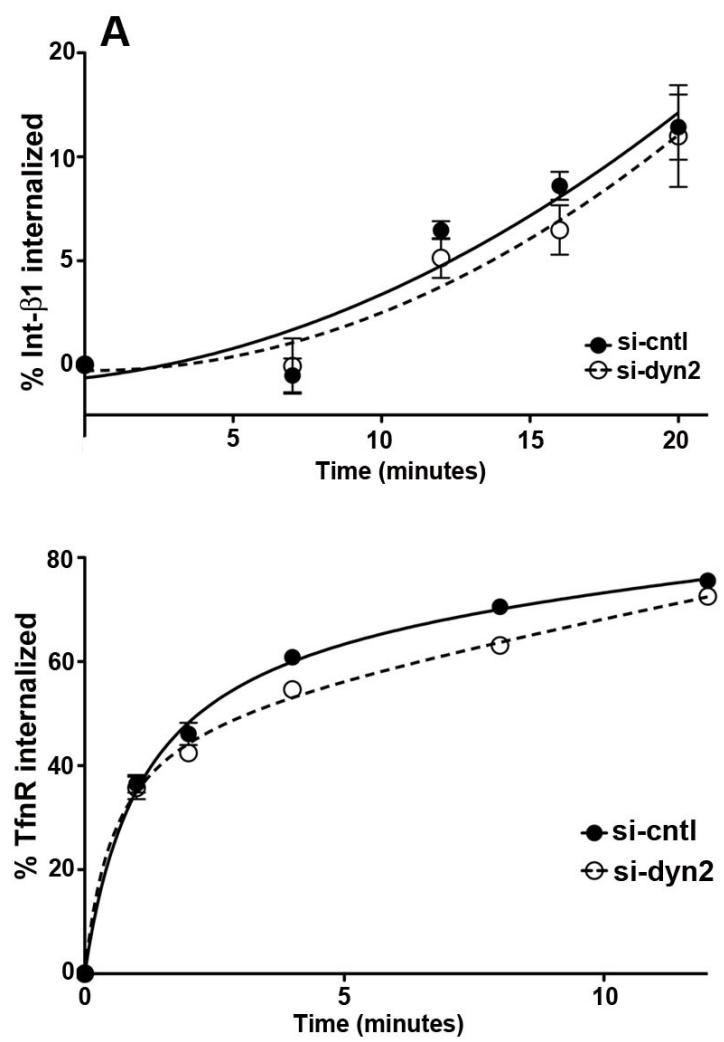

B
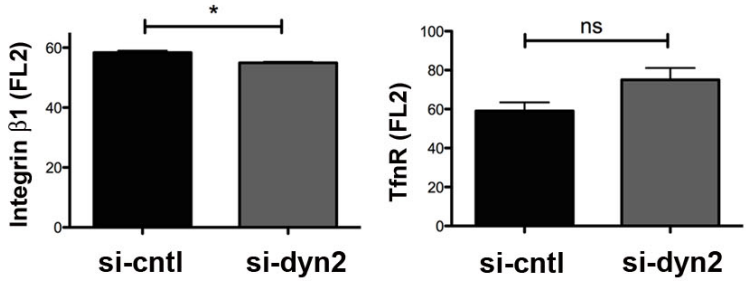

C

si-cntl si-dyn2
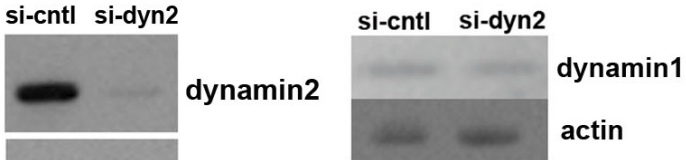


\section{Dynamin2 is enriched in the distal region of advancing lamellipodia}

To determine how dynamin2 influences actomyosin networks of the lamellum, we visualized GFP-WT-dynamin2 (GFP-WT-dyn2aa) in living U2-OS cells either depleted or not of dynamin2. As expected from previous studies (McNiven et al., 2000; Razidlo et al., 2013; Schafer et al., 2002), when expressed at low amounts, GFP-WT-dyn2 associated with lamellipodial actin networks and with dynamic $\alpha$-actinin-rich foci (Fig. 3A, Movie 2). Interestingly, GFP-WT-dyn2 was enriched in the distal-most region of the lamellipodial actin network, within $0.5 \mu \mathrm{m}$ of the cell edge. Moreover, the association of GFP-WT-dyn2 with the cell edge was discontinuous, being most enriched on advancing, as opposed to retracting, lamellipodial protrusions as visualized in movies and kymographs (Fig. 3B, Movie 2). GFP-WT-dyn2 was transiently associated with lamellipodial F-actin undergoing retrograde flow and with ruffles and filopodia that collapsed rearward and disassembled with the lamellipodial network (Fig. 3C, Movie 3). Away from the lamellipod, GFP-WT-dyn2 appeared transiently at diffraction-limited punctae that are likely sites of clathrin-mediated endocytosis (Movie 2) (Merrifield et al., 2002). GFP-WT-dyn2 was also dynamically associated with intracellular structures, most likely endosomes (not shown). In contrast with GFP-WT-dyn2, mCh-dynamin1 was primarily diffuse in the cytoplasm and appeared at transient, diffraction-limited punctae, but was not associated with lamellipodial actin networks (Fig. 4). The dynamic association of GFP-WT-dyn2 with the distal-most region of advancing lamellipodial Factin networks supports a role for dynamin2 in regulating the filaments that comprise those networks. 
Interactions of its C-terminal, proline-rich domain (PRD) were implicated in targeting dynamin2 to growth factor-induced ruffles and to lamellipodia (Krueger et al., 2003; McNiven et al., 2000). Dynamin2 binds components of lamellipodial F-actin, including the F-actin binding protein, cortactin, and the Rac-GEF, Vav1, via interactions of its PRD and the SH3 domains of cortactin or Vav1 (McNiven et al., 2000; Razidlo et al., 2013). Dynamin2 also binds actin filaments directly in vitro via a basic region in the dynamin2 stalk (Gu et al., 2010). To determine the mechanisms by which dynamin 2 is recruited to lamellipodial F-actin, we examined the spatial distribution and dynamics of mutant forms of fluorescent protein-tagged dynamin2 expressed in dyn2-depleted cells. Specifically, we examined GFP-dynamin2 lacking the PRD (GFP-dyn2- $\Delta \mathrm{PRD})$ and mCh-dynamin2 in which 5 lysine residues in the middle domain (K 414, 415, 419, 421 and 426) previously implicated in F-actin binding, were mutated to glutamic acid (mCh-dyn2- $\left.\mathrm{K}_{5} \mathrm{E}_{5}\right)(\mathrm{Gu}$ et al., 2010). Whereas dyn2- $\mathrm{K}_{5} \mathrm{E}_{5}$ binds F-actin with reduced affinity, it is reported to function during receptor-mediated endocytosis (Gu et al., 2010). Consistent with its reported endocytic activity, $\mathrm{mCh}$-dyn2- $\mathrm{K}_{5} \mathrm{E}_{5}$ was detected at transient, diffraction-limited punctae that are likely sites of endocytosis or endomembranes undergoing fission (not shown).

When expressed at low amounts in U2-OS cells previously depleted of endogenous dynamin 2 , mCh-dyn $2-\mathrm{K}_{5} \mathrm{E}_{5}$ was primarily diffusely distributed and not enriched at the distal lamellipod (Fig. 3A). Timelapse movies showed that regions within the lamellipod were transiently enriched in mCh-dyn2- $\mathrm{K}_{5} \mathrm{E}_{5}$, some of which moved rearward a short distance before disappearing (Movie 4). These transient regions of increased mCh-dyn2$\mathrm{K}_{5} \mathrm{E}_{5}$ fluorescence likely result from local increased cytoplasmic volume. Thus, we conclude that recruitment of dynamin2 to the distal lamellipod depends on basic amino 
acid residues in the stalk domain, presumably via direct interactions with actin filaments. Expression of $\mathrm{mCh}$-dyn2- $\mathrm{K}_{5} \mathrm{E}_{5}$ also promoted the formation of transient $\alpha$-actinindecorated cable-like structures that flowed rearward within the lamellipod (Fig. 3A, Movie 4), indicating that this mutant dynamin2 might rescue some of the effects of dyn2depletion on actomyosin formation (see below).

As expected from studies of fixed cells (McNiven et al., 2000; Orth et al., 2002), GFP-dyn2- $\triangle \mathrm{PRD}$ was diffusely distributed and conspicuously absent from lamellipodia when expressed in dyn2-depleted cells (Fig. 3A and Movie 5). As was observed with mCh-dyn2- $\mathrm{K}_{5} \mathrm{E}_{5}$, GFP-dyn2- $\triangle \mathrm{PRD}$ accumulated transiently in regions proximal to the cell periphery that may represent regions of increased cell thickness. Further back from the lamellipod, GFP-dyn2- $\triangle \mathrm{PRD}$ associated with transient, diffraction-limited punctae that are likely sites of endocytosis or endomembranes (not shown). Thus, interactions by both the C-terminal PRD and basic regions within its stalk are required to target dynamin2 to the distal edge of advancing lamellipodial protrusions. Moreover, the enrichment of dynamin2 within lamellipodia, but not with lamellar actin networks, supports a role for dynamin2 in regulating lamellipodial F-actin. 
Figure 3: Targeting dynamin2 to the distal lamellipod depends on direct interactions with F-actin and the C-terminal PRD.

(A) Single-frame images from movies of dyn2-depleted U2-OS cells transiently expressing mCh- $\alpha$-actinin and either GFP-wt-dyn2 or GFP-dyn2- $\Delta$ PRD, or mCh-dyn2$\mathrm{K}_{5} \mathrm{E}_{5}$ and GFP- $\alpha$-actinin, as indicated (see Movie 2). (The images of mCh-dyn2-K5E5 and GFP- $\alpha$-actinin were pseudocolored as green and red, respectively, to match other images displayed in this panel.)

(B) Representative kymographs depicting the spatial and temporal distribution of GFPWT-dyn2 in dyn2-depleted U2-OS cells.

(C) GFP-dyn2 associated transiently with filopodia and ruffles that collapsed rearward into the lamellipodia (see Movie 3). 
Figure. 3: Targeting dynamin2 to the distal lamellipod depends on direct interactions with F-actin and the C-terminal PRD.

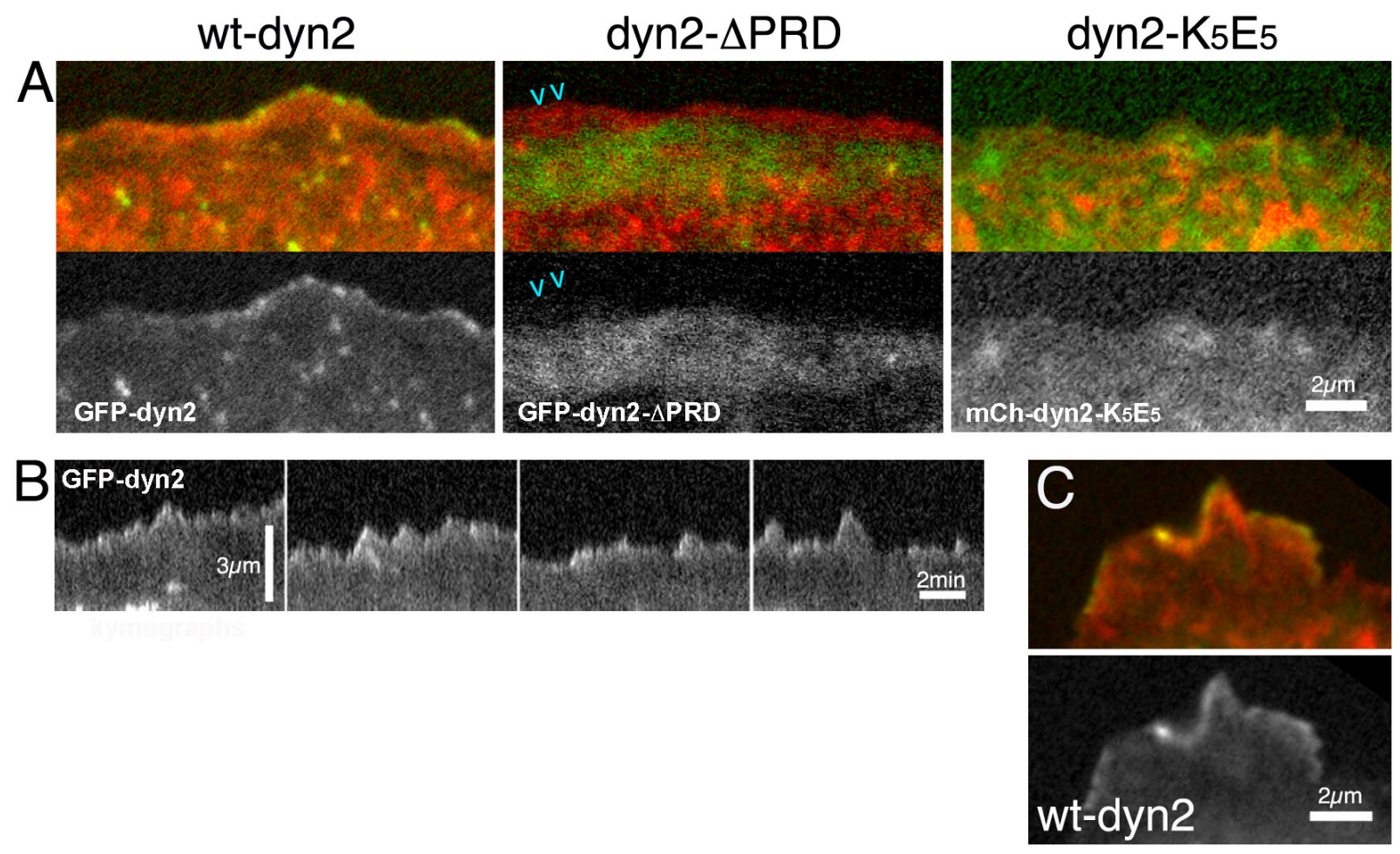


Figure 4: Dynamin1 is detected at diffraction-limited cytoplasmic punctae but not at the lamellipodia.

Single frames from two 5-frame movies of dyn2-depleted U2OS cells transiently expressing mCherry-dynamin1. Even though predominantly cytoplasmic, mCherrydynamin1 localizes to diffraction-limited puncta, but unlike dynamin2, is not present at the distal edge of the lamellipodia. 
Figure 4: Dynamin1 is detected at diffraction-limited cytoplasmic punctae but not at the lamellipodia.

mCherry-dynamin1
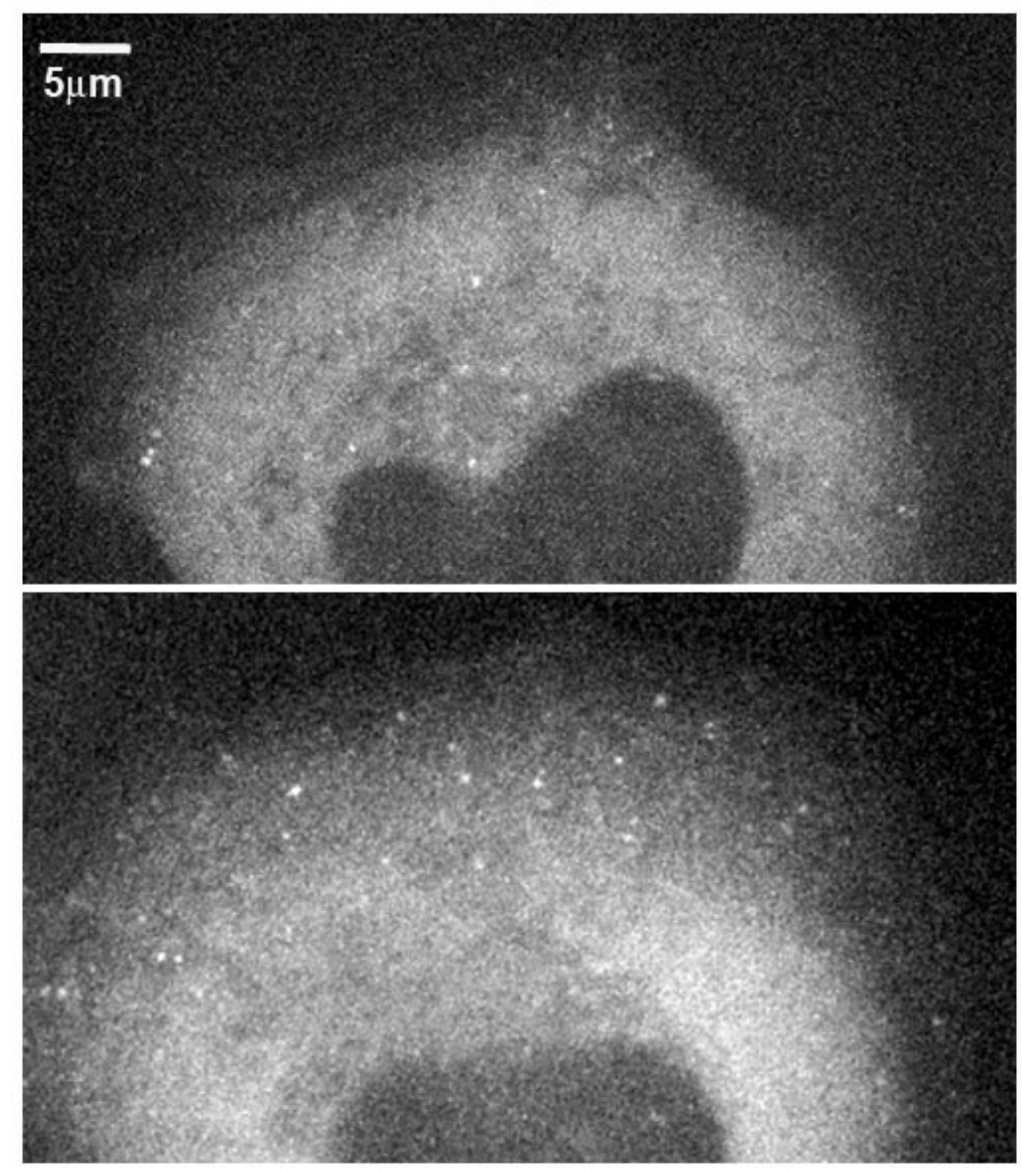


\section{Dynamin2 influences actin filament organization in lamellipodia}

To determine how dynamin2 influences lamellipodial actin networks, we examined the spatiotemporal dynamics of GFP- $\alpha$-actinin within lamellipodia. GFP- $\alpha$-actinin, rather than a fluorescent probe for actin, was used because it identified a subset of actin filaments within lamellipodial networks, some of which comprise actin filaments of lamellar actomyosin (Hotulainen and Lappalainen, 2006). GFP- $\alpha$-actinin was most enriched in the distal lamellipod of control siRNA-treated cells and flowed rearward at a rapid rate expected for a component of lamellipodial actin networks (Movie 6). The spatiotemporal distribution of GFP- $\alpha$-actinin within lamellipodial actin networks of control cells was non-homogeneous, as visualized in kymographs as distinct, diagonally oriented stripes (Fig. 5A, arrows). Stripes depicted the lamellipodial retrograde flow and frequently, but not always, coincided with retracting protrusions. Stripes were most intense at the cell periphery, becoming less intense closer to the boundary of the lamellipod and lamellum, indicating that the $\alpha$-actinin-enriched F-actin had disassembled and/or that GFP- $\alpha$-actinin had dissociated during retrograde flow. The loss of GFP- $\alpha-$ actinin contributed to the appearance of a distinct boundary between the lamellipod and lamellum where the retrograde flow slowed abruptly. Occasionally, stripes of GFP- $\alpha-$ actinin transited this boundary and moved into the lamellum but this event was rare in cells treated with control siRNAs. We interpret the stripes apparent in kymographs of lamellipodial actin networks as representing subsets of $\alpha$-actinin-enriched actin filaments undergoing retrograde flow.

In contrast, the spatiotemporal distribution of GFP- $\alpha$-actinin within lamellipodia of dynamin2-depleted cells appeared homogeneous; distinct stripes of GFP- $\alpha$-actinin were 
not prominent in kymographs (Fig. 5A and Movie 6). Notably, the boundary between the lamellipod and lamellum was not distinct in dyn2-depleted cells. In some cases, $\alpha$ actinin-decorated F-actin appeared to flow directly from the lamellipod to the lamellum. To quantitatively assess the spatiotemporal distribution of GFP- $\alpha$-actinin in lamellipodia of U2-OS cells, we measured the standard deviation of GFP- $\alpha$-actinin fluorescence intensity within the lamellipodial region of kymographs (Fig. 5B). The standard deviation of GFP- $\alpha$-actinin fluorescence within this region was significantly lower for dynamin2-depleted cells than for control siRNA-treated cells. Importantly, expression of mCh-WT-dynamin2 in dyn2-depleted cells restored the spatiotemporal distribution of GFP- $\alpha$-actinin, as determined by the increased standard deviation of GFP- $\alpha$-actinin fluorescence (Fig. 5B). We conclude that the distinct spatiotemporal distributions of GFP- $\alpha$-actinin within lamellipodia of control and dyn2-depleted cells reflect distinct actin filament architectures within lamellipodia under these two conditions. We further suggest that, through its actions on actin filaments of the distal lamellipod, dynamin2 remodels actin filaments that ultimately give rise to nascent actomyosin and transverse arcs of the lamellum several microns away.

Dynamin2 also influenced the distribution of cortactin, another component of lamellipodial actin networks. Immunolabeling fixed cells with anti-cortactin revealed that cortactin was enriched in the lamellipodia of dyn2-depleted cells when compared with control cells, an effect that was not due to increased cellular levels of cortactin (Fig. 6 A, B), lending additional support for a function of dynamin2 in organizing lamellipodial actin networks. However, in contrast with recent findings with pancreatic tumor cells (Razidlo et al., 2013), the cellular levels of another lamellipodial actin 
regulator that binds dynamin2 directly, the Rac1 GEF, Vav1, were unchanged in dyn2depleted U2-OS cells (Fig. 6C). 
Figure 5: Dynamin2 influences the spatiotemporal organization of $\alpha$-actinin in lamellipodia.

(A) Representative kymographs obtained from timelapse movies of GFP- $\alpha$-actinin in lamellipodia of control, dyn2-depleted and mCh-wt-dyn2 rescued, dyn2-depleted cells. Stripes (arrows) in the kymographs represent subsets of lamellipodial F-actin enriched in GFP- $\alpha$-actinin. Scale bar: $1 \mu \mathrm{m}$

(B) Graph depicting the standard deviation of GFP- $\alpha$-actinin fluorescence intensity within the lamellipodial region of control, dyn2-depleted and mCh-wt-dyn2 rescued, dyn2-depleted cells. ** $\mathrm{p}<0.005$; ns, not significant. Data were obtained from two independent experiments. 
Figure 5: Dynamin2 influences the spatiotemporal organization of $\alpha$-actinin in lamellipodia.

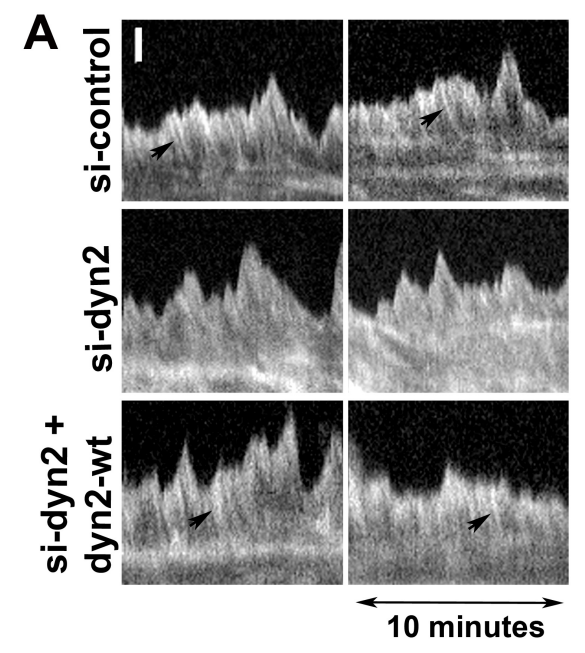

B

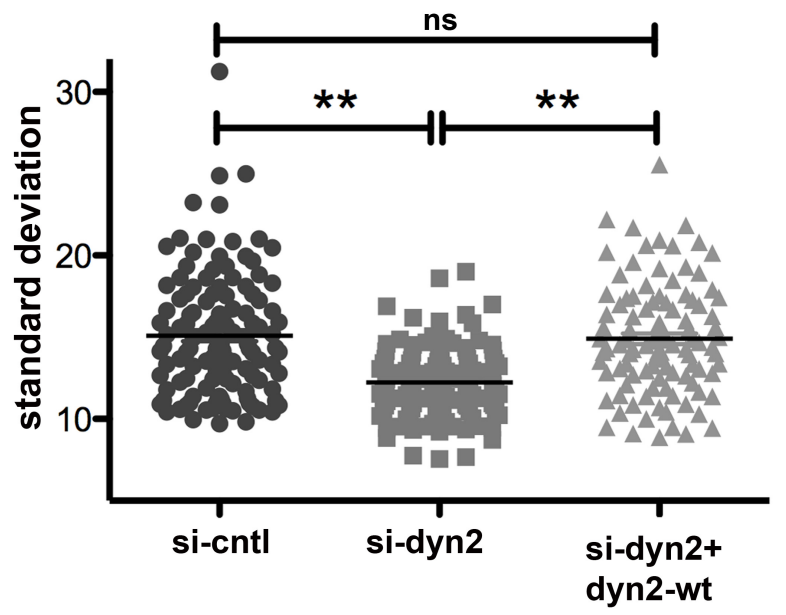


Figure 6: The spatial distribution of cortactin within lamellipodia and in the cytoplasm is altered in dyn2-depleted cells.

(A) Representative images of control and dyn2-depleted fixed cells immunolabeled with anti-cortactin (red) and Alexa488-phalloidin (cyan-blue). (B) Plotted are the integrated fluorescence intensities/area for anti-cortactin immunostaining in control and dyn2depleted U2-OS cells. Each point represents data obtained from a single cell and data were collected from at least 30 cells in each group.

(C) Cell lysates from equal numbers of control and dyn2-depleted cells were subjected to electrophoresis in $10 \%$ polyacrylamide gels followed by transfer to nitrocellulose for detection of cortactin and Vav1/2. Expression of cortactin and Vav1/2 were not perturbed in dyn2-depleted cells. A cell lysate from Jurkat cells was used as a positive control for the anti-Vav antibody. 
Figure 6: The spatial distribution of cortactin within lamellipodia and in the cytoplasm is altered in dyn2-depleted cells.
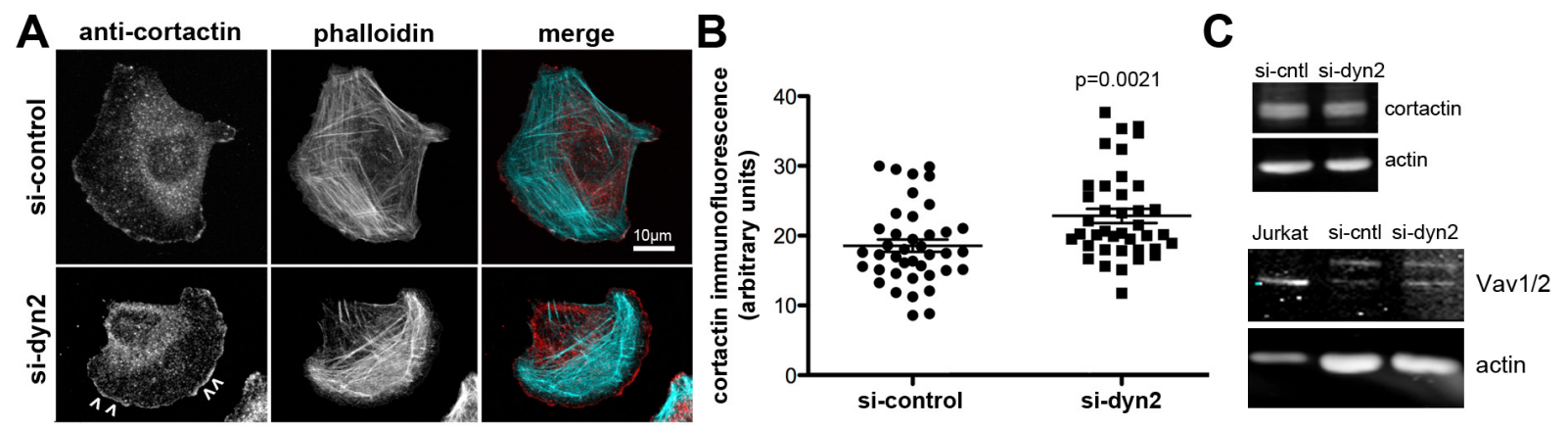


\section{Dynamin2-dependent filament remodeling influences the linkage of lamellar actomyosin and focal adhesions}

The increased retrograde flow exhibited by transverse arcs in dynamin2-depleted cells could arise via several mechanisms, including signaling that increases activation of myosin II, the motor powering lamellar retrograde flow, decreased assembly of focal adhesions that engage transverse arcs and slow their retrograde flow or decreased coupling between adhesions and transverse arcs. To determine if dynamin 2 influences signaling downstream of RhoA, leading to activation of myosin II, we examined phosphorylation of two effectors of active RhoA, MLC2 and ADF/cofilin, in control and dynamin2-depleted cells. Neither the total amount of MLC2 or of ADF/cofilin, nor the amount of phosphorylated form of each protein, was altered in lysates obtained from dyn2-depleted U2-OS cells compared with control cells (Fig. 7A). Although we have not ruled out that these RhoA effectors may be selectively activated locally and transiently within lamellipodia via a dyn2-dependent process, we conclude that dynamin2 does not likely increase lamellar retrograde flow via effects on signaling pathways that activate myosin II. In addition, levels of focal adhesion kinase (FAK) and activated, phosphoFAK were unchanged in dyn2-depleted cells.

To determine if dyn2 influences the focal adhesions that engage transverse arcs during retrograde flow, we examined the dynamics and morphology of newly formed focal adhesions in cells expressing GFP-paxillin. Nascent adhesions in U2-OS cells elongate rapidly, forming elongated structures, some of which became wider as they matured. In contrast, focal adhesions formed near the leading edge in dyn2-depleted cells remained thin, resulting in a higher length/width ratio (Fig. 7B). Nonetheless, the rates of 
adhesion assembly and disassembly were identical in control and dyn2-depleted cells (Fig. 7C). Thus, dynamin2 influences adhesion morphology in U2-OS cells, but not adhesion dynamics.

Focal adhesions associate with transverse arcs and slow the rate of lamellar retrograde flow (Alexandrova et al., 2008; Burnette et al., 2011). $\alpha$-Actinin is one link between adhesions and flowing actin filaments (Brown et al., 2006; Hu et al., 2007; Kanchanawong et al., 2010). To explore a role for dynamin 2 in regulating the linkage between transverse arcs and adhesions, we observed mCh- $\alpha$-actinin and GFP-paxillin as new adhesions formed in U2-OS cells (Fig. 7D). Timelapse movies of control siRNAtreated U2-OS cells revealed that nascent adhesions formed within lamellipodia and elongated centripetally at a rapid rate (Movie 7). mCh- $\alpha$-actinin accumulated at the proximal end of elongating adhesions (Fig. 7E and Movie 7). Kymographs obtained from pixel-wide lines drawn along newly formed adhesions indicated that $\mathrm{mCh}-\alpha$-actinin accumulated at the proximal end of adhesions in control cells within $\sim 6$ min (Fig. 7E). In contrast, whereas mCh- $\alpha$-actinin accumulated at many newly formed adhesions in dyn2depleted cells by $\sim 10.5 \mathrm{~min}, \mathrm{mCh}-\alpha$-actinin had not accumulated at $\sim 38 \%$ of adhesions by $<20 \mathrm{~min}$, the duration of our movies (Fig. 7E). Instead, amorphous mCh- $\alpha$-actinindecorated structures flowed from the lamellipodia to the lamellar region, bypassing adhesions (Fig. 7E, lower panels and Movie 7). Thus, we conclude that the rapid rate of lamellar actomyosin retrograde flow observed in dyn2-depleted cells results from weak engagement of poorly organized, discontinuous arcs with adhesions. We propose that dynamin2 remodels actin filaments within lamellipodia for optimal assembly of arcs and their engagement at adhesions. 


\section{Figure 7: Dynamin2 influences coupling of transverse arcs and adhesions.}

(A) Western blots depict levels of total and phosphorylated MLC2, ADF/cofilin and FAK in control and dynamin2-depleted U2OS cells. Images were obtained using a Li-COR imaging system and numbers denote the ratio of phospho-to-total protein for each pair; actin is the loading control.

(B) Graphs depict the length, width and length/width of nascent focal adhesions in control and dyn2-depleted cells. Measurements were obtained from adhesions formed near the cell edge in cells transiently expressing GFP-paxillin and $\mathrm{mCh}-\alpha$-actinin. ** $\mathrm{p}<0.005$; ns, not significant.

(C) Rates of focal adhesion assembly (left panel) and disassembly (right panel) are plotted as described in Experimental methods. The number of adhesions analyzed in control and in dyn2-depleted cells was 19 and 46, respectively.

(D) Single images from representative movies of control and dyn2-depleted cells expressing GFP-paxillin and mCh- $\alpha$-actinin (see Movie 7)

(E) Kymographs obtained from pixel-wide lines drawn along the length of elongating adhesions from timelapse movies described in panel D. In control cells, $\alpha$-actinin (red) accumulated at the proximal end of elongating adhesions (green) within about 6 min (arrowheads); $\alpha$-actinin appeared later at adhesions in dyn2-depleted cells and its association with adhesions was transient. 
Figure 7: Dynamin2 influences coupling of transverse arcs and adhesions.

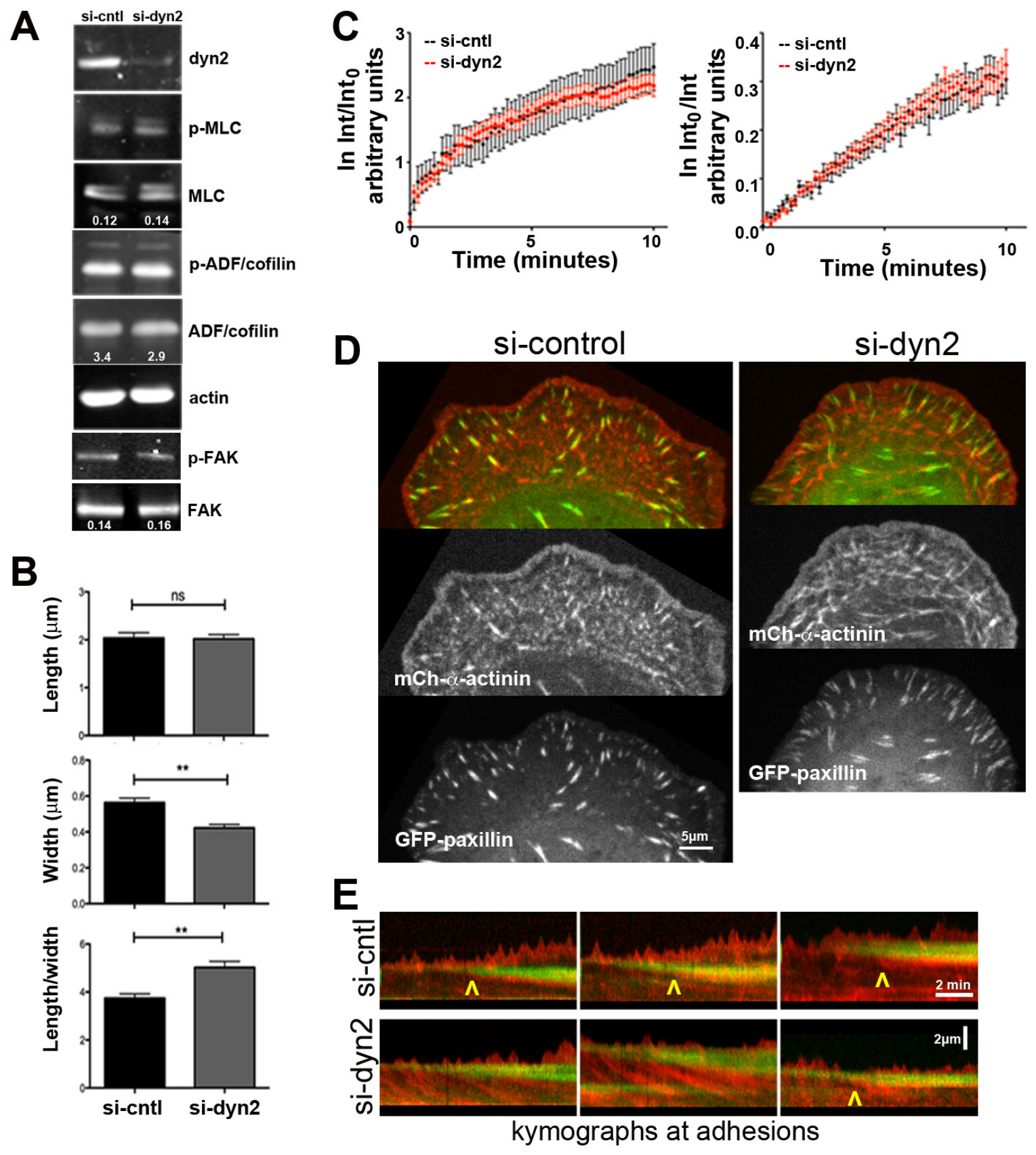


Actin filament remodeling by dynamin2 requires interactions with actin filaments, interactions of the PRD and finely tuned GTP hydrolysis.

To determine the biochemical activities of dynamin2 required for remodeling lamellipodial actin networks and for assembly of transverse arcs, we expressed mutant forms of dynamin2 in dyn2-depleted cells and assessed their ability to restore the spatiotemporal distribution of GFP- $\alpha$-actinin in lamellipodia and lamellar retrograde flow. We first examined mutant dynamin2 proteins defective in interactions with actin filaments or lacking the dynamin2 PRD. Since the C-terminal PRD targets dynamin2 to lamellipodial F-actin, is was not surprising that mCh-dyn2- $\triangle \mathrm{PRD}$ neither restored the spatiotemporal distribution of GFP- $\alpha$-actinin in lamellipodia nor the rate of retrograde flow of transverse arcs in dyn2-depleted cells (Fig. 8A and Fig. 9A). Other features of lamellar actomyosin networks characteristic of dyn2-depleted cells, such as misshaped, frequently appearing GFP-MLC2 punctae and a lack of continuous $\alpha$-actinin-decorated cables were also apparent in cells expressing mCh-dyn2- $\triangle \mathrm{PRD}$ (Movies 8 and 5). These findings highlight the importance of dynamin2 PRD interactions for its activities on actin filaments.

To determine if direct interactions of dynamin 2 and actin filaments were required for actin filament remodeling, we expressed the actin-binding deficient mutant protein, $\mathrm{mCh}$ dyn2- $\mathrm{K}_{5} \mathrm{E}_{5}$ in dyn2-depleted cells. Although $\mathrm{mCh}-\mathrm{dyn} 2-\mathrm{K}_{5} \mathrm{E}_{5}$ was diffusely distributed within lamellipodia and not enriched at the cell edge (Fig. 3C), its expression restored the spatiotemporal distribution of $\alpha$-actinin within lamellipodia as determined from the standard deviation of GFP- $\alpha$-actinin fluorescence in kymographs of dynamic lamellipods (Fig. 8B). Examination of time-lapse movies of cells expressing mCh-dyn2- $\mathrm{K}_{5} \mathrm{E}_{5}$ 
revealed that the increased standard deviation of $\alpha$-actinin fluorescence resulted from prominent $\alpha$-actinin-decorated cables that formed as lamellipodial protrusions retracted (Movie 4). As they flowed retrogradely, the cable structures slowed and deformed slightly as they encountered adhesions, but did not cohesively engage them. Consistent with this observation, a fast rate of lamellar retrograde flow typical of that observed in dyn2-depleted cells was retained in cells expressing mCh-dyn2- $\mathrm{K}_{5} \mathrm{E}_{5}$ (Fig. 9B). In addition, as was observed in dyn2-depleted cells, punctae of GFP-MLC2 appeared more frequently and were aberrantly shaped in cells expressing mCh-dyn2- $\mathrm{K}_{5} \mathrm{E}_{5}$ (Movie 8). We conclude that dyn2- $\mathrm{K}_{5} \mathrm{E}_{5}$ partially restores dynamin2 function to dyn2-depleted cells, most likely by inducing formation of $\alpha$-actinin-decorated cables. This activity of dyn2$\mathrm{K}_{5} \mathrm{E}_{5}$ could result from interactions of $\mathrm{mCh}-\mathrm{dyn} 2-\mathrm{K}_{5} \mathrm{E}_{5}$ with PRD-interacting proteins, such as cortactin, which binds actin filaments and promotes filament bundling in the presence of dynamin2 in vitro (Mooren et al., 2009). Moreover, since dyn2- $\mathrm{K}_{5} \mathrm{E}_{5}$ was reported to retain basal and assembly-stimulated GTP hydrolysis in vitro and to support clathrin-mediated endocytosis in vivo ( $\mathrm{Gu}$ et al., 2010), partial restoration of dyn2 function in cells expressing $\mathrm{mCh}-\mathrm{dyn} 2-\mathrm{K}_{5} \mathrm{E}_{5}$ supports the conclusion that dynamin2 regulates actin filaments independently from its role in endocytosis. In summary, both direct and indirect interactions of dynamin2 with actin filaments are required for dynamin2-dependent actin remodeling that contributes to formation and dynamics of transverse actomyosin arcs.

We previously showed that GTP hydrolysis by dynamin2, in the presence of cortactin, remodels bundled actin filaments in vitro (Mooren et al., 2009). Dynamin2 GTPase activity was also implicated in controlling F-actin recruitment and disassembly at 
sites of clathrin-mediated endocytosis (Taylor et al., 2012) and in regulating apical actomyosin in epithelial cells (Chua et al., 2009). To determine if the rate of GTP hydrolysis by dynamin2 influences lamellipodial and lamellar actin networks, we expressed in dyn2-depleted cells two mutant dynamin2 proteins, dyn2-T141A and dyn2S61D, that exhibit increased and decreased, respectively, rates of basal and lipidstimulated GTP hydrolysis (Song et al., 2004). Like GFP-WT-dyn2, GFP-dyn2-T141A and GFP-dyn2-S61D were targeted to the distal edge of advancing lamellipodial protrusions (Fig. 10A and Movie 9). Interestingly, compared with GFP-WT-dyn2, association of GFP-dyn2-T141A with the distal lamellipod appeared more transient, whereas GFP-dyn2-S61D was persistently associated with the distal lamellipod and flowed rearward a short distance with lamellipodial F-actin (Movie 9). Moreover, protrusions in cells expressing GFP-dyn2-S61D extended more slowly and were longerlived compared to those in cells expressing GFP-WT-dyn2 or GFP-dyn2-T141A (Fig. 10B), suggesting that the rate of GTP hydrolysis by dynamin2 acting on lamellipodial actin filaments influences actin network organization and its dynamic behavior. Consistent with this conclusion, neither GTP-hydrolysis defective mutant dynamin2 proteins restored lamellipodial filament organization, as assessed from the spatiotemporal distribution of GFP- $\alpha$-actinin (Fig. 8 C,D), or lamellar retrograde flow (Fig. 9 C-D). We conclude that the rate of GTP hydrolysis by dynamin2 associated with lamellipodial actin networks influences actin filament organization, the protrusive behavior driven by that network and, further downstream, lamellar actomyosin. Because dyn2-T141A supported receptor mediated endocytosis (Song et al., 2004) but did not restore dynamin2dependent activities on actin filaments, the conclusion that dynamin2 remodels 
lamellipodial actin networks independently of its function in receptor-mediated endocytosis is reinforced. 
Figure 8: Dynamin2-dependent organization of GFP- $\alpha$-actinin within lamellipodia depends on F-actin binding, the PRD and finely tuned GTP hydrolysis.

(A-D) Plotted is the standard deviation of lamellipodial GFP- $\alpha$-actinin fluorescence intensity obtained from kymographs in control, dyn2-depleted and dyn2-depleted cells reexpressing (A) mCh-dyn2- $\triangle \mathrm{PRD}$, (B) mCh-dyn2- $\mathrm{K}_{5} \mathrm{E}_{5}$, (C) mCh-dyn2-T141A and (D) mCh-dyn2-S61D. ** $\mathrm{p}<0.005$; ns, not significant. Data were obtained from two independent experiments that yielded similar results. 
Figure 8: Dynamin2-dependent organization of GFP- $\alpha$-actinin within lamellipodia depends on F-actin binding, the PRD and finely tuned GTP hydrolysis.
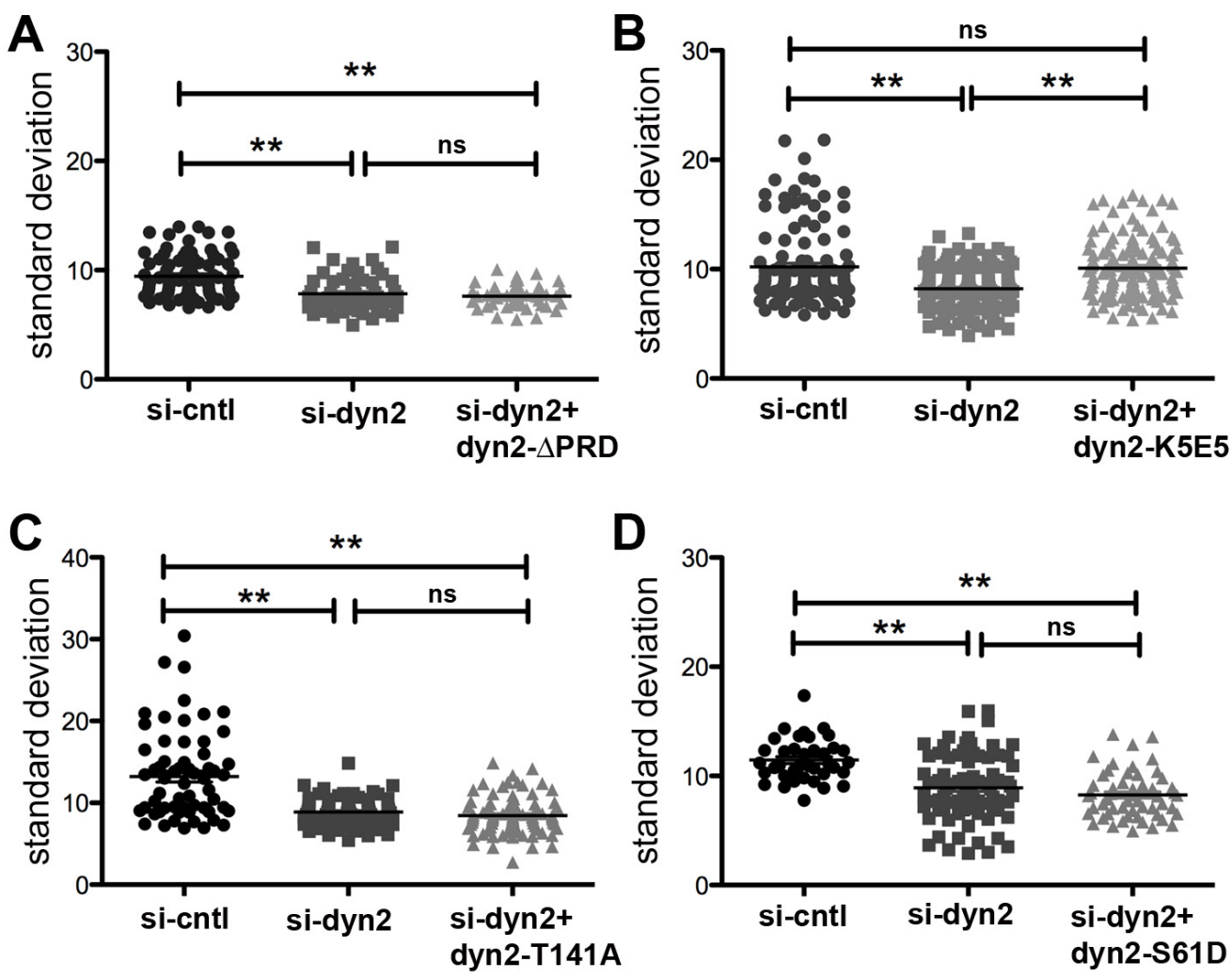
Figure 9: Dynamin2-dependent regulation of lamellar actomyosin retrograde flow depends on F-actin binding, the PRD and its rate of GTP hydrolysis.

(A-D) Plotted is the rate of retrograde flow of GFP-MLC2-containing arcs in control, dyn2-depleted and dyn2-depleted cells transiently expressing (A) mCh-dyn2- $\Delta \mathrm{PRD},(\mathrm{B})$ mCh-dyn2-K $\mathrm{E}_{5}$ (C) mCh-dyn2-T141A and (D) mCh-dyn2-S61D. ** $\mathrm{p}<0.005$; ns, not significant. Data were obtained from two independent experiments that yielded similar results. 
Figure 9: Dynamin2-dependent regulation of lamellar actomyosin retrograde flow depends on F-actin binding, the PRD and its rate of GTP hydrolysis.
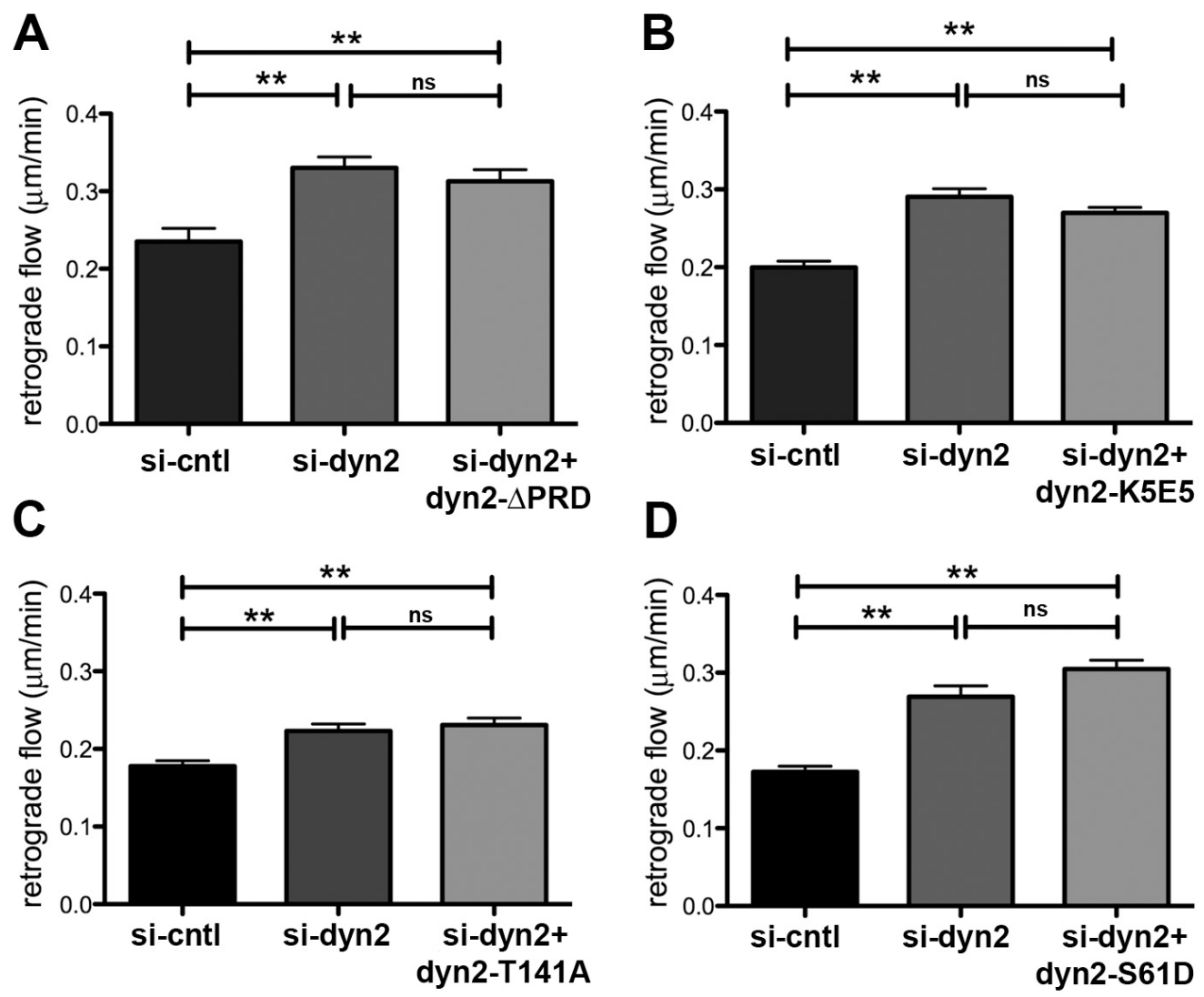
Figure 10: GTP hydrolysis by dynamin2 influences protrusion dynamics.

(A) Single images from movies of dyn2-depleted U2-OS cells re-expressing GFP-dyn2T141A or GFP-dyn2-S61D, along with mCh- $\alpha$-actinin (see Movie 9). Like GFP-WTdyn2, both GTPase mutants localize to the distal edge of the lamellipodia. Scale bar: 2 $\mu \mathrm{m}$.

(B) Plotted is the rate of advance, distance advanced and duration of lamellipodial protrusions in dyn2-depleted U2-OS cells and in depleted cells transiently expressing WT-dyn2, dyn2-T141A or dyn2-S61D. ** $\mathrm{p}<0.005, * \mathrm{p}<0.05$. Protrusions observed in 10 kymographs/cell were quantified; the number of cells analyzed for each condition was as follows: si-dyn2 (5); si-dyn2+wt-dyn2 (8); si-dyn2+dyn2-T141A (6) and si-dyn2+dyn2S61D (5). 
Figure 10: GTP hydrolysis by dynamin2 influences protrusion dynamics.
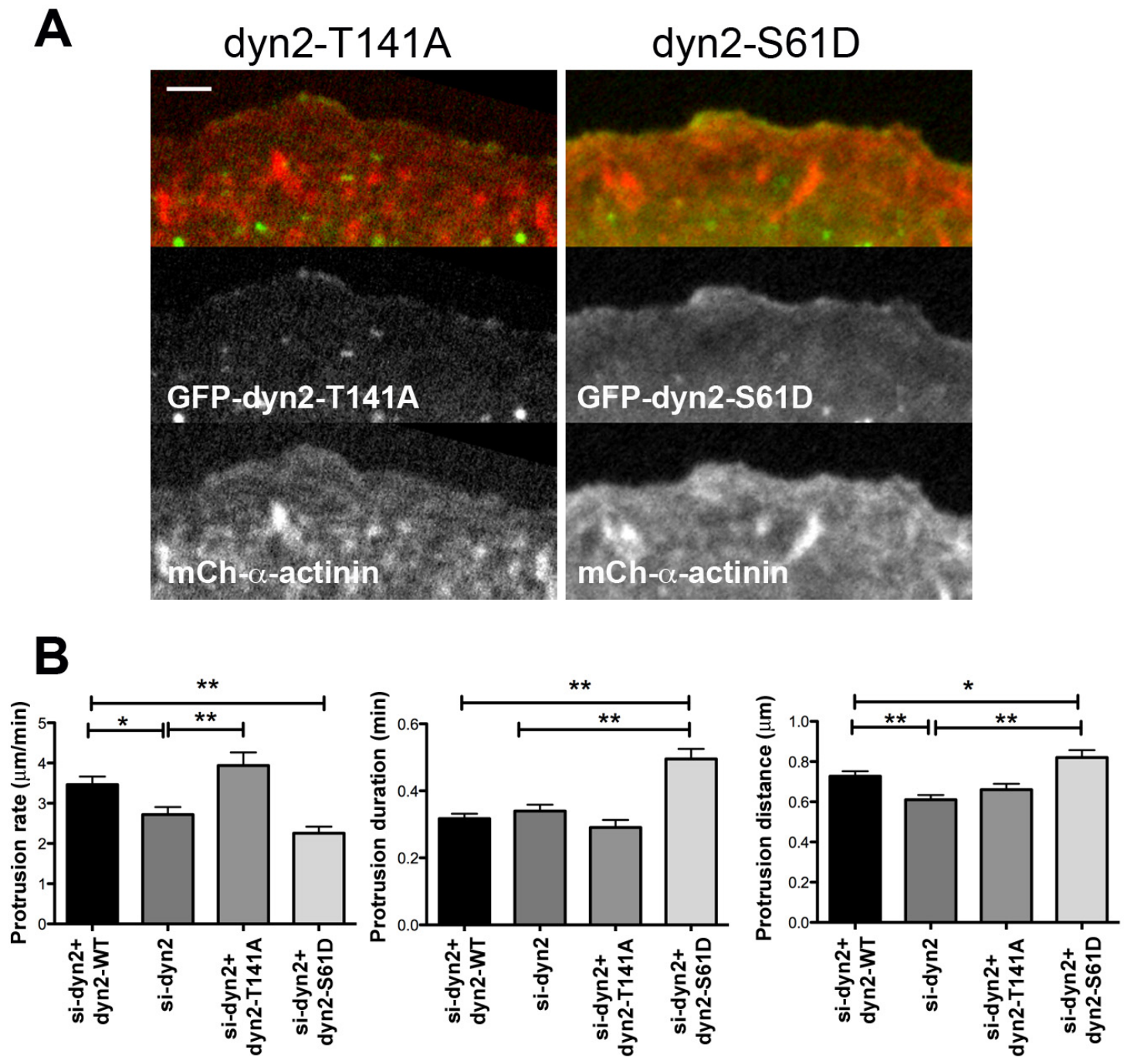


\section{Discussion}

We provide evidence that dynamin2, which is enriched at the distal lamellipodial actin network in U2-OS cells, regulates the assembly and dynamics of lamellar actomyosin situated several microns away. Loss of dynamin 2 perturbed assembly of nascent actomyosin of transverse arcs and increased arc retrograde flow via a mechanism that weakened the coupling of arcs to focal adhesions. Within lamellipodia, dynamin2 influenced protrusive behavior, regulated the distributions of $\alpha$-actinin and cortactin and defined the interface between the lamellipod and lamellum. The ability of dynamin 2 to organize lamellipodial F-actin, and thereby orchestrate lamellar actomyosin assembly depends on its rate of GTP hydrolysis, interactions via the dynamin2 PRD and F-actin binding by the dyn 2 stalk, but does not depend on membrane remodeling by dynamin 2 during receptor-mediated endocytosis. We propose that actin filament remodeling by dynamin 2 within the distal lamellipod integrates and coordinates the lamellipodial and lamellar actin networks and their coupling to cell-ECM adhesions to establish a coherent global actin cytoskeleton.

\section{Integrating actin networks in migrating cells}

Considerable evidence implicates dynamin2 in regulating the actin cytoskeleton but the functional significance of this activity in living cells remains unknown (Chua et al., 2009; Kruchten and McNiven, 2006; Krueger et al., 2003; Lee and De Camilli, 2002; Menon and Schafer, 2013; Mooren et al., 2009; Ochoa et al., 2000). Collectively, our experiments demonstrate how dynamin2 influences actin networks in U2-OS cells. First, dynamin 2 associated selectively with actin filaments situated at the distal-most edge of advancing protrusions. As expected from previous reports (McNiven et al., 2000), the 
dyn2 PRD was required for targeting dynamin2 to the lamellipod; we now report that Factin binding via the dyn 2 stalk is also involved. Like other actin binding proteins, the dynamin2 stalk may preferentially bind segments of actin filaments enriched in ATPactin subunits, which are abundant at the distal leading edge (Pollard and Borisy, 2003). Alternatively, as proposed for selective binding of Arp2/3 complex to filaments at the leading edge (Risca et al., 2012), compressive forces acting on actin filaments pushing against the plasma membrane may bend filaments, creating a structural template optimal for binding the dynamin2 stalk. Finally, interactions of dynamin2 and cortactin, which stabilizes Arp2/3 complex at branched filament junctions (Weaver et al., 2001), could cooperatively reinforce interactions of dynamin 2 with actin filaments subjacent to the plasma membrane.

Second, dynamin2 organizes actin filaments within lamellipodia that specify F-actin networks of lamella. Formation of transverse arcs is a highly coordinated process in which bipolar filaments of myosin II and $\alpha$-actinin-crosslinked lamellipodial F-actin coassemble (Hotulainen and Lappalainen, 2006; Shutova et al., 2012; Verkhovsky et al., 1995). Recent work has shown that components of lamellar actomyosin arise, in part, from actin filaments of the lamellipod (Anderson et al., 2008; Burnette et al., 2011; Hotulainen and Lappalainen, 2006; Nemethova et al., 2008; Tojkander et al., 2011). Although we did not observe filament organization directly, the observation that dynamin2 influenced the spatiotemporal distribution within lamellipodia of the actin filament crosslinker, $\alpha$-actinin, suggests a role for dynamin 2 in specifying actin filament architecture. We propose that dyn2-dependent actin filament remodeling generates within lamellipodia a filament template organized by $\alpha$-actinin that is optimal for co- 
assembly with myosin II. This idea is consistent with one model for assembly of actomyosin of transverse arcs that posits annealing of myosin II-containing structures and $\alpha$-actinin-decorated filaments derived from lamellipodial actin networks (Hotulainen and Lappalainen, 2006; Tojkander et al., 2011). Recent ultrastructural analyses of nascent actomyosin reforming after blebbistatin washout also showed that bundled filaments were preferred substrates for myosin II assembly (Shutova et al., 2012). In the absence of dynamin2, nascent actomyosin structures appeared uncontrolled. MLC2 punctae appeared more frequently and were misshaped, often merging with other punctae, expanding into irregular shapes or disassembling altogether as they flowed rearward. We predict these behaviors result because the appropriate actin filament templates are not generated in the absence of dynamin2. Consistent with this idea, distinct, continuous cable-like structures that emerge as transverse arcs engage focal adhesions also did not form in dyn2-depleted cells. Instead $\alpha$-actinin was associated with discontinuous, amorphous structures that flowed retrogradely at a fast rate. The enhanced accumulation of cortactin within lamellipodia of dyn2-depleted cells may stabilize branched filament junctions (Weaver et al., 2001), further interfering with filament alignment required to create a bundled filament template.

Finally, the distinct interface between the lamellipodium and lamellum depended on dynamin2. A hallmark of the lamellipodium-lamellum interface is the transition between fast and slow retrograde flow (Alexandrova et al., 2008; Ponti et al., 2004). Focal adhesions forming in the lamellipod are thought to trigger the transition from fast to slow flow by interacting with flowing actin filaments (Alexandrova et al., 2008; Burnette et al., 2011; Shemesh et al., 2009). In U2-OS cells, lamellar network retrograde flow 
slowed in dyn2-depleted cells, but not to the extent observed in control cells. Even though adhesion dynamics were relatively unperturbed in cells lacking dynamin2, adhesion width was reduced and coupling of lamellar actomyosin to adhesions near the leading edge, as viewed using the marker mCh- $\alpha$-actinin, was delayed. These observations support the idea that dynamin2 regulates formation of a structural template ideal for actomyosin assembly, formation of transverse arcs and their productive engagement with focal adhesions. Although adhesion assembly and maturation depends on contractile activity of myosin II (Chrzanowska-Wodnicka and Burridge, 1996; Totsukawa et al., 2000; Vicente-Manzanares et al., 2007), recent evidence suggests that actin filament organization within the lamellipod and the lamellum is also important for adhesion maturation (Choi et al., 2008; Oakes et al., 2012). Thus, we propose that dynamin2 integrates the actin networks of lamellipodia and lamellae and the dynamic linkages between lamellar actin networks and cell-ECM adhesions.

\section{Dynamin as an actin filament-remodeling machine}

How does dynamin2 direct actin filament architecture? Previous biochemical studies showed that dynamin proteins exhibit at least two actions on actin filaments in vitro: bundling and severing. Dynamin1 bundles actin filaments in vitro ( $\mathrm{Gu}$ et al., 2010), whereas filament bundling by dynamin2 was greatly enhanced by cortactin (Mooren et al., 2009). Notably, GTP hydrolysis by dynamin2 and cortactin remodeled bundled filaments, creating loosely bundled filaments from tightly associated filaments and also severing filaments (Mooren et al., 2009). The loosely bundled filaments formed upon GTP hydrolysis by dynamin 2 may be ideal templates for crosslinking by $\alpha$-actinin, an elongated molecule $\sim 37 \mathrm{~nm}$ in length (Meyer and Aebi, 1990). Alternatively, loosely 
bundled filaments generated by dynamin 2 and cortactin may be refractory to crosslinking by $\alpha$-actinin via a mechanism that involves interactions of dynamin 2 with the nonreceptor kinase, focal adhesion kinase (FAK) (Wang et al., 2011). FAK phosphorylates $\alpha$-actinin, decreasing its affinity for F-actin (Izaguirre et al., 2001). A FAK-dynamin2 complex at the leading edge could create a bundled template in which $\alpha$-actinin-mediated crosslinks are selectively limited due to elevated phospho- $\alpha$-actinin. Although activation of FAK was not perturbed in dyn2-depleted cells and GFP-FAK was not enriched at the distal lamellipodia (Fig. 11), whether or not dynamin2's interaction with FAK alters the level of phospho- $\alpha$-actinin in lamellipodia remains to be investigated.

$\alpha$-Actinin is a promiscuous actin filament crosslinking protein, capable of crosslinking filaments in vitro as either linear, bundled arrays or as meshworks of randomly-oriented filaments (Courson and Rock, 2010). Once crosslinked as bundles, $\alpha$ actinin-bound filaments remain bundled, even under conditions of rapid crosslinker exchange. Filament severing by dynamin2 GTPase could promote filament bundling within dense meshworks by shortening filaments, allowing them to diffuse into positions favorable for crosslinking as linear arrays (Maciver et al., 1991). Alternatively, dynamin2 GTPase-dependent filament severing could potentiate selective filament turnover within the lamellipod, particularly at the distal leading edge where actin depolymerization factors are less abundant (Pollard and Borisy, 2003; Svitkina and Borisy, 1999). In support of this idea, dynamin2 GTPase-dependent filament remodeling promoted cofilin-mediated filament severing in vitro (Mooren et al., 2009). The fact that $\alpha$-actinin-decorated F-actin is enriched in the distal lamellipod of control cells, but 
distributed more broadly throughout the lamellipod of dynamin2-depleted cells, suggests dyamin 2 limits the population of actin filaments that becomes organized by $\alpha$-actinin.

Filament severing by dynamin 2 could promote actin polymerization by creating new actin filament barbed ends. However, we obtained no evidence that dynamin2 promotes assembly of F-actin structures in U2-OS cells. Protrusions occurred frequently, albeit at a slightly decreased rate, in dyn2-depleted cells compared with control U2-OS cells, and dynamin2 expression did not alter the cellular level of the Rac1 GEF, Vav1 (Fig. 6), which was recently shown to promote protrusive activity in invasive tumor cells (Razidlo et al., 2013). In U2-OS cells, dynamin2 expression influenced the overall organization of F-actin as visualized in phalloidin-stained fixed cells and the extent of myosin IIA and $\alpha$ actinin associated with F-actin was increased in cells lacking dynamin2 (Mooren et al., 2009). On the other hand, a role for dynamin2 in promoting actin filament turnover is supported from the accumulation of F-actin at clathrin-coated structures in cells either lacking dynamin or expressing mutant dynamins that perturb fission activity (Ferguson et al., 2009; Taylor et al., 2012). Whether or not filament remodeling by dynamin2 potentiates filament turnover at clathrin-coated structures is unknown.

Dynamin2 is emerging as a multi-functional scaffolding protein that may assemble a diverse array of actin regulators. Hence, its actions on actin filaments may be cell- and context-specific. A general function for dynamin2 in specifying new filament architectures or potentiating altered dynamic properties to actin networks could account for the diverse effects on the actin cytoskeleton observed when dynamin 2 activity is disrupted in different cellular contexts. Our data includes a comprehensive set that indicates actin filament remodeling by dynamin 2 is not controlled by its actions during 
endocytic processes. However, given dynamin's expanding roles in secretion and exocytosis, endocytic recycling and signaling, dynamin2 may act to remodel both actin filaments and cellular membranes coordinately as it contributes to whole cell behaviors. 
Figure. 11: GFP-FAK does not localize to the distal lamellipod

Single frame from a movie of a control U2-OS cell co-transfected with GFP-FAK and mCherry- $\alpha$-actinin. Scale bar: $2 \mu \mathrm{m}$. 
Figure. 11: GFP-FAK does not localize to the distal lamellipod

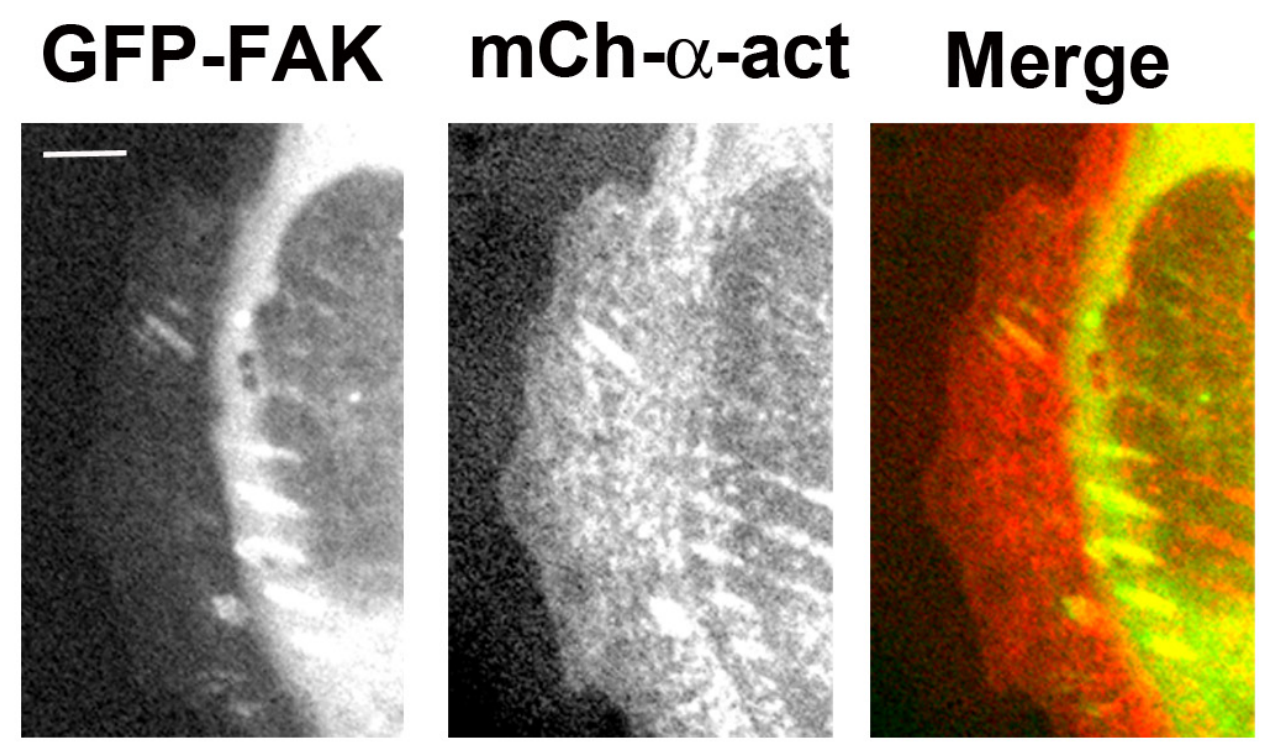




\section{Materials and Methods}

Plasmids and antibodies

Plasmids to express WT or mutant FP-tagged rat dynamin2aa (dyn2) were constructed from dyn2-wt pCR3.1 (a gift from Mark McNiven, Mayo Clinic, Rochester, MN). Site-directed mutagenesis was accomplished using the QuickChange II kit (Agilent Technologies, Santa Clara, CA, USA) and primers from Integrated DNA Technologies, Inc. (Coralville, IA, USA) or Eurofins MWG Operon (Huntsville, AL, USA). To prepare dyn2- $\mathrm{K}_{5} \mathrm{E}_{5}$, three rounds of mutagenesis reactions were performed, introducing K-to-E mutations at positions $414,415,419,421$ and 426 (numbers for rat dyn2 cDNA). cDNAs were subcloned into the EcoR1/HindIII sites of either pmCherry-C1 (pmCh-C1) or pEGFP-C1 (Clontech, Mountain View, CA, USA) using standard procedures. GFP-WTdyn2 was a gift from Dr. K. Nakayama (Kasai et al., 1999). Plasmids for expressing GFP$\alpha$-actinin, mCh- $\alpha$-actinin and GFP-MLC2 were from Dr. Rick Horwitz (University of Virginia, Charlottesville, VA), GFP-paxillin was from Dr. Martin Schwartz (Yale School of Medicine, New Haven, CT) and mCh-dynamin1 was from Dr. David Castle (University of Virginia, Charlottesville, VA). Primary antibodies used in this study are listed in Table S1. Secondary antibodies were obtained from Jackson ImmunoResearch (West Grove, PA, USA), LI-COR Biosciences (Lincoln, Nebraska, USA) and Invitrogen (Grand Island, NY, USA).

Cell culture and transfections

U2-OS cells (ATCC HTB-96, Manassas, VA, USA) were grown in Dulbecco's modified Eagle's medium (DMEM) supplemented with $10 \%$ fetal bovine serum (FBS), glutamine, sodium pyruvate and non-essential amino acids. Dynamin2 was depleted using siGenome 
siRNAs (Dharmacon, Lafayette, CO, USA) targeting human dynamin2 (D2-18, AGUCCUACAUCAACACGAA (catalog number D-004007-18)); a non-targeting siRNA was the control siRNA (catalog number D-001210-05). siRNAs (2 $\mu \mathrm{g}$ ) were delivered into cells using an Amaxa Nucleofector II, program X-001, and nucleofection kit V (Amaxa Biosystems, Lonza, Basel, Switzerland) according to the manufacturer's protocol. Cells were used $48 \mathrm{~h}$ after nucleofection. Cells were transfected with plasmids (1-1.2 $\mu \mathrm{g}$ ) using FuGene HD (Promega, Fitchburg, WI, USA) as directed by the manufacturer and cultured $\sim 16$ hours before imaging.

$\underline{\text { Live-cell imaging }}$

U2-OS cells were plated on glass-bottomed dishes (MatTek Corporation, Ashland, MA, USA) coated with $2 \mu \mathrm{g} / \mathrm{ml}$ fibronectin (Sigma, St. Louis, MO, USA) for 34 hours; 30 min prior to imaging, media was replaced with 'movie media' (phenol-red free MEM supplemented with 10\% FBS, glutamine and 20mM HEPES, pH 7). Confocal microscopy was performed using a Zeiss microscope with a Nakagawa spinning disc, $63 \mathrm{X}$ oil objective lens, lasers to excite GFP (488nm) and mCherry $(587 \mathrm{~nm})$, dual 512x512 EM X2-CCD cameras and/or ORCA R2 camera (Hamamatsu, Bridgewater, NJ, USA), all controlled by Metamorph software. Cells were maintained at $37^{\circ} \mathrm{C}$ during microscopy. For image stacks, 5-7 images, each $\sim 0.5 \mu \mathrm{m}$ apart, were obtained at each time point. A Definite Focus controller (Carl Zeiss, Jena, Germany) was used to maintain focus during single focal plane collection.

Quantitation of receptor internalization

Internalization of transferrin receptor (TfnR) or $\beta 1$-integrin was quantified using flow cytometry as described (Moravec et al., 2012), with slight modifications. $8 \times 10^{4}$ 
cells/well in a 12-well plate were washed with ice-cold HyQCCM1 media containing 0.2\% BSA and 0.01M HEPES, pH $7.0(\mathrm{HyQ})$ and incubated on an ice-slurry $1 \mathrm{~h}$ with either PE-conjugated mouse anti-integrin $\beta 1$ or PE-conjugated mouse anti-TfnR diluted in HyQ. After three washes in ice-cold HyQ, warm HyQ was added and cells incubated at $37^{\circ} \mathrm{C}$ for varying periods of time. At each time point, warm media was replaced with ice-cold HyQ and incubation 30 min with AlexaFluor-647 donkey anti-mouse to label any surface-associated anti-receptor antibody. After washing in HyQ, cells were detached using Accutase (Innovative Cell technologies, San Diego, CA, USA) and analyzed by flow cytometry (FACSCalibur, Becton Dickinson, Franklin Lakes, NJ, USA). The fluorescence intensities of PE-conjugated anti-receptor antibody (which represents the total surface-associated pool before internalization) and of AlexaFluor647-conjugated antibody (which binds any receptor-PE-antibody complex remaining on the surface) at each time point were normalized to the respective intensity at zero time to determine relative amounts of total receptor and surface-bound receptor. Antibody remaining on the surface after internalization was obtained from the ratio of normalized AlexaFluor647-labeled antibody fluorescence/normalized PE-conjugated antibody fluorescence for each time point; the inverse of this value represents the relative amount of internalized receptor.

Quantitative western blotting

Whole cell lysates were prepared from $2 \times 10^{5}$ cells suspended in $200 \mu l$ hot 2XSDS sample buffer, heated at $95^{\circ} \mathrm{C}$ and stored at $-20^{\circ} \mathrm{C}$. Lysates to quantify phosphorylated proteins were prepared from $4 \times 10^{5}$ cells plated on $60 \mathrm{~mm}$ dishes; cells were washed 2X with ice-cold PBS and lysed with $250 \mu$ l hot 1X SDS containing Roche 
protease inhibitor cocktail, $0.2 \mathrm{mM}$ PMSF and $2 \mathrm{mM}$ sodium orthovanadate. Cells were scraped from the dish, boiled at $95^{\circ} \mathrm{C}$, and stored at $-20^{\circ} \mathrm{C}$. Whole cell lysates were subjected to electrophoresis in 10-15\% polyacrylamide gels (Laemmli, 1970); proteins in gels were transferred to nitrocellulose for detection using immunoblotting. Blots were blocked in 5\% milk or LiCOR blocking buffer (for phospho-proteins) (Lincoln, Nebraska, USA), incubated with primary antibodies and IR-dye-conjugated secondary antibodies and quantitatively analyzed using a Li-COR Odyssey infrared imager (Lincoln, Nebraska, USA).

Quantitative analysis of lamellar network retrograde flow, the spatiotemporal distribution of GFP- $\alpha$-actinin in lamellipodia and lamellipodial protrusion dynamics

Timelapse movies of GFP-MLC2-expressing cells were collected at 10s/frame over 20 min using an EM-CCD camera. Lamellar retrograde flow was determined from kymographs of pixel-wide lines perpendicular to the cell edge at regions where transverse arcs formed using the kymography Plugin in ImageJ (National Institute of Health, Bethesda, MD, USA). The rate of lamellar retrograde flow was determined from the slope of lines in the kymographs that represent individual segments of transverse arcs. Data were exported to MS Excel and GraphPad Prism for analysis and graphing.

Timelapse movies of cells expressing GFP- $\alpha$-actinin were collected at $5 \mathrm{~s} /$ frame over 10 min using an ORCA R2 camera. Kymographs were generated as described above from regions where lamellipodia were actively protruding. The lamellipodial region of each kymograph was selected as a region-of-interest (ROI) and the standard deviation of the intensity of GFP fluorescence within the ROI was determined using the Measure function in ImageJ. 
To analyze protrusion dynamics, kymographs were plotted from pixel-wide wide lines perpendicular to the cell edge along regions exhibiting protrusive activity. Kymographs were analyzed using a custom program to obtain the rate of extension, distance and duration of protrusions as described (Bear et al., 2002; Cai et al., 2007; Hinz et al., 1999).

\section{Analysis of focal adhesions}

The length and width of newly formed focal adhesions were determined from movies of cells expressing GFP-paxillin using measurement tools available in ImageJ. To quantify the rates of assembly and disassembly of GFP-paxillin-containing adhesions, timelapse movies were collected at $10 \mathrm{~s} /$ frame over $20 \mathrm{~min}$. Adhesion assembly rate was determined from the slope of graphs of the change in GFP-paxillin fluorescence intensity vs. time, plotted as $\operatorname{Ln}\left(\right.$ intensity $\left(\mathrm{I}_{\mathrm{t}}\right)$ /initial intensity $\left(\mathrm{I}_{0}\right)$ ) as described (Bryce et al., 2005). Similarly, adhesion disassembly rate was determined from graphs of Ln(initial intensity $\left(\mathrm{I}_{0}\right)$ /intensity $\left.\left(\mathrm{I}_{\mathrm{t}}\right)\right)$ vs. time. Background fluorescence obtained from a region adjacent to adhesions was subtracted before analysis. 


\section{References}

Alexandrova, A.Y., Arnold, K., Schaub, S., Vasiliev, J.M., Meister, J.J., Bershadsky, A.D., and Verkhovsky, A.B. (2008). Comparative dynamics of retrograde actin flow and focal adhesions: formation of nascent adhesions triggers transition from fast to slow flow. PLoS One 3, e3234.

Anderson, T.W., Vaughan, A.N., and Cramer, L.P. (2008). Retrograde flow and myosin II activity within the leading cell edge deliver F-actin to the lamella to seed the formation of graded polarity actomyosin II filament bundles in migrating fibroblasts. Mol Biol Cell 19, 5006-5018.

Aratyn-Schaus, Y., Oakes, P.W., and Gardel, M.L. (2011). Dynamic and structural signatures of lamellar actomyosin force generation. Mol Biol Cell 22, 1330-1339.

Bear, J.E., Svitkina, T.M., Krause, M., Schafer, D.A., Loureiro, J.J., Strasser, G.A., Maly, I.V., Chaga, O.Y., Cooper, J.A., Borisy, G.G., et al. (2002). Antagonism between Ena/VASP proteins and actin filament capping regulates fibroblast motility. Cell 109, 509-521.

Brown, C.M., Hebert, B., Kolin, D.L., Zareno, J., Whitmore, L., Horwitz, A.R., and Wiseman, P.W. (2006). Probing the integrin-actin linkage using high-resolution protein velocity mapping. J Cell Sci 119, 5204-5214.

Bryce, N.S., Clark, E.S., Leysath, J.L., Currie, J.D., Webb, D.J., and Weaver, A.M. (2005). Cortactin promotes cell motility by enhancing lamellipodial persistence. Curr Biol 15, 1276-1285.

Burnette, D.T., Manley, S., Sengupta, P., Sougrat, R., Davidson, M.W., Kachar, B., and Lippincott-Schwartz, J. (2011). A role for actin arcs in the leading-edge advance of migrating cells. Nat Cell Biol 13, 371-381.

Burridge, K., and Wittchen, E.S. (2013). The tension mounts: stress fibers as forcegenerating mechanotransducers. J Cell Biol 200, 9-19.

Cai, L., Marshall, T.W., Uetrecht, A.C., Schafer, D.A., and Bear, J.E. (2007). Coronin 1B coordinates Arp2/3 complex and cofilin activities at the leading edge. Cell 128, 915-929.

Choi, C.K., Vicente-Manzanares, M., Zareno, J., Whitmore, L.A., Mogilner, A., and Horwitz, A.R. (2008). Actin and alpha-actinin orchestrate the assembly and maturation of nascent adhesions in a myosin II motor-independent manner. Nat Cell Biol 10, 1039-1050

Chrzanowska-Wodnicka, M., and Burridge, K. (1996). Rho-stimulated contractility drives the formation of stress fibers and focal adhesions. J Cell Biol 133, 1403-1415. 
Chua, J., Rikhy, R., and Lippincott-Schwartz, J. (2009). Dynamin 2 orchestrates the global actomyosin cytoskeleton for epithelial maintenance and apical constriction. Proc Natl Acad Sci U S A 106, 20770-20775.

Courson, D.S., and Rock, R.S. (2010). Actin cross-link assembly and disassembly mechanics for alpha-Actinin and fascin. J Biol Chem 285, 26350-26357.

Ferguson, S.M., Raimondi, A., Paradise, S., Shen, H., Mesaki, K., Ferguson, A., Destaing, O., Ko, G., Takasaki, J., Cremona, O., et al. (2009). Coordinated actions of actin and BAR proteins upstream of dynamin at endocytic clathrin-coated pits. Dev Cell 17, 811-822.

Gardel, M.L., Sabass, B., Ji, L., Danuser, G., Schwarz, U.S., and Waterman, C.M. (2008). Traction stress in focal adhesions correlates biphasically with actin retrograde flow speed. J Cell Biol 183, 999-1005.

Gardel, M.L., Schneider, I.C., Aratyn-Schaus, Y., and Waterman, C.M. (2010). Mechanical integration of actin and adhesion dynamics in cell migration. Annu Rev Cell Dev Biol 26, 315-333.

Giannone, G., Mege, R.M., and Thoumine, O. (2009). Multi-level molecular clutches in motile cell processes. Trends Cell Biol 19, 475-486.

Gu, C., Yaddanapudi, S., Weins, A., Osborn, T., Reiser, J., Pollak, M., Hartwig, J., and Sever, S. (2010). Direct dynamin-actin interactions regulate the actin cytoskeleton. Embo J 29, 3593-3606.

Hamao, K., Morita, M., and Hosoya, H. (2009). New function of the proline rich domain in dynamin-2 to negatively regulate its interaction with microtubules in mammalian cells. Exp Cell Res 315, 1336-1345.

Heath, J.P. (1983). Behaviour and structure of the leading lamella in moving fibroblasts. I. Occurrence and centripetal movement of arc-shaped microfilament bundles beneath the dorsal cell surface. J Cell Sci 60,331-354.

Henmi, Y., Tanabe, K., and Takei, K. (2011). Disruption of microtubule network rescues aberrant actin comets in dynamin2-depleted cells. PLoS One 6, e28603.

Hinz, B., Alt, W., Johnen, C., Herzog, V., and Kaiser, H.W. (1999). Quantifying lamella dynamics of cultured cells by SACED, a new computer- assisted motion analysis. Exp Cell Res 251, 234-243.

Hotulainen, P., and Lappalainen, P. (2006). Stress fibers are generated by two distinct actin assembly mechanisms in motile cells. J Cell Biol 173, 383-394. 
Hu, K., Ji, L., Applegate, K.T., Danuser, G., and Waterman-Storer, C.M. (2007). Differential transmission of actin motion within focal adhesions. Science 315, 111115.

Izaguirre, G., Aguirre, L., Hu, Y.P., Lee, H.Y., Schlaepfer, D.D., Aneskievich, B.J., and Haimovich, B. (2001). The cytoskeletal/non-muscle isoform of alpha-actinin is phosphorylated on its actin-binding domain by the focal adhesion kinase. J Biol Chem 276, 28676-28685.

Kanchanawong, P., Shtengel, G., Pasapera, A.M., Ramko, E.B., Davidson, M.W., Hess, H.F., and Waterman, C.M. (2010). Nanoscale architecture of integrin-based cell adhesions. Nature 468, 580-584.

Kasai, K., Shin, H.W., Shinotsuka, C., Murakami, K., and Nakayama, K. (1999). Dynamin II is involved in endocytosis but not in the formation of transport vesicles from the trans-Golgi network. J Biochem (Tokyo) 125, 780-789.

Kruchten, A.E., and McNiven, M.A. (2006). Dynamin as a mover and pincher during cell migration and invasion. J Cell Sci 119, 1683-1690.

Krueger, E.W., Orth, J.D., Cao, H., and McNiven, M.A. (2003). A dynamin-cortactinArp2/3 complex mediates actin reorganization in growth factor-stimulated cells. Mol Biol Cell 14, 1085-1096.

Laemmli, U.K. (1970). Cleavage of structural proteins during the assembly of the head of bacteriophage T4. Nature 227, 680-685.

Lee, E., and De Camilli, P. (2002). Dynamin at actin tails. Proc Natl Acad Sci U S A 99, 161-166.

Maciver, S.K., Wachsstock, D.H., Schwarz, W.H., and Pollard, T.D. (1991). The actin filament severing protein actophorin promotes the formation of rigid bundles of actin filaments crosslinked with alpha-actinin. J Cell Biol 115, 1621-1628.

McNiven, M.A., Kim, L., Krueger, E.W., Orth, J.D., Cao, H., and Wong, T.W. (2000). Regulated interactions between dynamin and the actin-binding protein cortactin modulate cell shape. J Cell Biol 151, 187-198.

Menon, M., and Schafer, D.A. (2013). Dynamin: expanding its scope to the cytoskeleton. International Review of Cell and Molecular Biology 302, 188-219.

Merrifield, C.J., Feldman, M.E., Wan, L., and Almers, W. (2002). Imaging actin and dynamin recruitment during invagination of single clathrin-coated pits. Nat Cell Biol 4, 691-698. 
Meyer, R.K., and Aebi, U. (1990). Bundling of actin filaments by alpha-actinin depends on its molecular length. J Cell Biol 110, 2013-2024.

Mooren, O.L., Kotova, T.I., Moore, A.J., and Schafer, D.A. (2009). Dynamin2 GTPase and cortactin remodel actin filaments. J Biol Chem 284, 23995-24005.

Moravec, R., Conger, K.K., D'Souza, R., Allison, A.B., and Casanova, J.E. (2012). BRAG2/GEP100/IQSec1 interacts with clathrin and regulates alpha5beta1 integrin endocytosis through activation of ADP ribosylation factor 5 (Arf5). J Biol Chem 287, 31138-31147.

Naumanen, P., Lappalainen, P., and Hotulainen, P. (2008). Mechanisms of actin stress fibre assembly. J Microsc 231, 446-454.

Nemethova, M., Auinger, S., and Small, J.V. (2008). Building the actin cytoskeleton: filopodia contribute to the construction of contractile bundles in the lamella. J Cell Biol 180, 1233-1244.

Oakes, P.W., Beckham, Y., Stricker, J., and Gardel, M.L. (2012). Tension is required but not sufficient for focal adhesion maturation without a stress fiber template. J Cell Biol 196,363-374.

Ochoa, G.C., Slepnev, V.I., Neff, L., Ringstad, N., Takei, K., Daniell, L., Kim, W., Cao, H., McNiven, M., Baron, R., et al. (2000). A functional link between dynamin and the actin cytoskeleton at podosomes. J Cell Biol 150, 377-389.

Orth, J.D., Krueger, E.W., Cao, H., and McNiven, M.A. (2002). The large GTPase dynamin regulates actin comet formation and movement in living cells. Proc Natl Acad Sci U S A 99, 167-172.

Orth, J.D., and McNiven, M.A. (2003). Dynamin at the actin-membrane interface. Curr Opin Cell Biol 15, 31-39.

Pollard, T.D., and Borisy, G.G. (2003). Cellular motility driven by assembly and disassembly of actin filaments. Cell 112, 453-465.

Ponti, A., Machacek, M., Gupton, S.L., Waterman-Storer, C.M., and Danuser, G. (2004). Two distinct actin networks drive the protrusion of migrating cells. Science $305,1782-1786$.

Razidlo, G.L., Wang, Y., Chen, J., Krueger, E.W., Billadeau, D.D., and McNiven, M.A. (2013). Dynamin 2 Potentiates Invasive Migration of Pancreatic Tumor Cells through Stabilization of the Rac1 GEF Vav1. Dev Cell 24, 573-585. 
Risca, V.I., Wang, E.B., Chaudhuri, O., Chia, J.J., Geissler, P.L., and Fletcher, D.A. (2012). Actin filament curvature biases branching direction. Proc Natl Acad Sci U S A 109, 2913-2918.

Rossier, O.M., Gauthier, N., Biais, N., Vonnegut, W., Fardin, M.A., Avigan, P., Heller, E.R., Mathur, A., Ghassemi, S., Koeckert, M.S., et al. (2010). Force generated by actomyosin contraction builds bridges between adhesive contacts. EMBO J 29, 10551068.

Schafer, D.A., Weed, S.A., Binns, D., Karginov, A.V., Parsons, J.T., and Cooper, J.A. (2002). Dynamin2 and cortactin regulate actin assembly and filament organization. Curr Biol 12, 1852-1857.

Schwarz, U.S., and Gardel, M.L. (2012). United we stand: integrating the actin cytoskeleton and cell-matrix adhesions in cellular mechanotransduction. J Cell Sci 125, 3051-3060.

Shemesh, T., Verkhovsky, A.B., Svitkina, T.M., Bershadsky, A.D., and Kozlov, M.M. (2009). Role of focal adhesions and mechanical stresses in the formation and progression of the lamellipodium-lamellum interface [corrected]. Biophys J 97, 12541264.

Shutova, M., Yang, C., Vasiliev, J.M., and Svitkina, T. (2012). Functions of nonmuscle myosin II in assembly of the cellular contractile system. PLoS One 7, e40814.

Song, B.D., Leonard, M., and Schmid, S.L. (2004). Dynamin GTPase domain mutants that differentially affect GTP binding, GTP hydrolysis, and clathrin-mediated endocytosis. J Biol Chem 279, 40431-40436.

Svitkina, T.M., and Borisy, G.G. (1999). Arp2/3 complex and actin depolymerizing factor cofilin in dendritic organization and treadmilling of actin filament array in lamellipodia. Journal of Cell Biology 145, 1009-1026.

Tanabe, K., and Takei, K. (2009). Dynamic instability of microtubules requires dynamin 2 and is impaired in a Charcot-Marie-Tooth mutant. J Cell Biol 185, 939-948.

Taylor, M.J., Lampe, M., and Merrifield, C.J. (2012). A feedback loop between dynamin and actin recruitment during clathrin-mediated endocytosis. PLoS Biol 10, e1001302.

Thompson, H.M., Cao, H., Chen, J., Euteneuer, U., and McNiven, M.A. (2004). Dynamin 2 binds gamma-tubulin and participates in centrosome cohesion. Nat Cell Biol 6, 335-342.

Tojkander, S., Gateva, G., and Lappalainen, P. (2012). Actin stress fibers--assembly, dynamics and biological roles. J Cell Sci 125, 1855-1864. 
Tojkander, S., Gateva, G., Schevzov, G., Hotulainen, P., Naumanen, P., Martin, C., Gunning, P.W., and Lappalainen, P. (2011). A molecular pathway for myosin II recruitment to stress fibers. Curr Biol 21,539-550.

Totsukawa, G., Yamakita, Y., Yamashiro, S., Hartshorne, D.J., Sasaki, Y., and Matsumura, F. (2000). Distinct roles of ROCK (Rho-kinase) and MLCK in spatial regulation of MLC phosphorylation for assembly of stress fibers and focal adhesions in $3 \mathrm{~T} 3$ fibroblasts. J Cell Biol 150, 797-806.

Verkhovsky, A.B., Svitkina, T.M., and Borisy, G.G. (1995). Myosin II filament assemblies in the active lamella of fibroblasts: their morphogenesis and role in the formation of actin filament bundles. J Cell Biol 131, 989-1002.

Vicente-Manzanares, M., Zareno, J., Whitmore, L., Choi, C.K., and Horwitz, A.F. (2007). Regulation of protrusion, adhesion dynamics, and polarity by myosins IIA and IIB in migrating cells. J Cell Biol 176, 573-580.

Wang, Y., Cao, H., Chen, J., and McNiven, M.A. (2011). A direct interaction between the large GTPase dynamin-2 and FAK regulates focal adhesion dynamics in response to active Src. Mol Biol Cell 22, 1529-1538.

Weaver, A.M., Karginov, A.V., Kinley, A.W., Weed, S.A., Li, Y., Parsons, J.T., and Cooper, J.A. (2001). Cortactin promotes and stabilizes Arp2/3-induced actin filament network formation. Curr Biol 11,370-374. 


\section{Chapter 4}

\section{Discussion and Future Perspectives}


How actin networks in cells reorganize during processes like the assembly of actomyosin arcs, invagination of membrane during endocytosis or the formation and maintenance of cellular junctions is not completely understood. In this study, I identified a novel regulator of actomyosin assembly and organization, dynamin2. Dynamin2 localizes to the distal edge of the lamellipodia, close to the actin crosslinking protein $\alpha$ actinin. Based on this localization, I hypothesized that dynamin2 could influence the organization of lamellipodial actin which can then regulate actomyosin assembly. At the lamellipodia, dynamin2 influenced the spatiotemporal distribution of $\alpha$-actinin, suggesting that dynamin 2 could orchestrate the organization of lamellipodial actin networks to specify an actin architecture for cross-linking by $\alpha$-actinin. In the following sections, I will propose a model to explain how dynamin2 remodels lamellipodial actin networks.

Dynamin2 influenced assembly, organization and dynamics of lamellar actomyosin arcs. Dynamin2-depleted cells exhibited increased rate of appearance of myosin puncta at the lamellipod-lamellum boundary during assembly of nascent arcs. These arcs maintained a higher rate of retrograde flow, presumably due to defective engagement with newly forming focal adhesions. This research therefore uncovered a previously unappreciated function for dynamin2 in global actomyosin assembly and organization. Earlier work that implicated dynamin2 in actomyosin organization and contractility was based on experiments using fixed cells. My work used live-cell imaging to closely examine the dynamics of actomyosin assembly to better understand dynamin2's role in the process. 
Model for dynamin2-dependent regulation of actin networks

At the lamellipodia, actin networks exist in a branched, dendritic array and it is not well-understood whether and how lamellipodial actin gets remodeled to form lamellar actomyosin. Even though it has been shown that lamellipodial and lamellar actin are two separate populations (Ponti et al., 2004; Vallotton and Small, 2009), there is also empirical evidence suggesting that a part of the lamellipodial actin network contributes to formation of actomyosin arcs (Anderson et al., 2008; Burnette et al., 2011; Hotulainen and Lappalainen, 2006; Nemethova et al., 2008; Tojkander et al., 2011). Actomyosin arc formation involves length-wise association of $\alpha$-actinin-bundled actin filaments and myosin II, which starts at the lamellipod-lamellum interface and gets progressively aligned during lamellar retrograde flow (Hotulainen and Lappalainen, 2006). Hence, properly organized actin bundles might be required for efficient actomyosin assembly. One possible way the transition of the branched actin network organization at lamellipodia to that of a bundled network at lamella occurs is via localized severing of actin filaments at the lamellipodia that enables the filaments to diffuse more easily into a network of shorter parallel bundles cross-linked by proteins like $\alpha$-actinin (Maciver et al., 1991). I propose dynamin2 bundles and severs actin filaments at the distal lamellipodia. This actin remodeling by dynamin 2 could set up an effective template for lamellar actin assembly by generating actin filaments in the right orientation for bundling at the lamellipodia by crosslinking proteins like $\alpha$-actinin (Fig. 1A). An efficiently organized lamellar network can then associate with nascent adhesions forming along the lamellipod-lamellum boundary (Fig. 1A). 
GFP-dyn2-WT localizes to advancing edges of protrusions; there is evidence in vitro that dynamin functions as an actin bundling protein (Gu et al., 2010; Mooren et al., 2009). Therefore I propose that dynamin 2 bundles actin along advancing protrusions (Fig. 1B). Dynamin2-dependent GTP hydrolysis then induces a conformational change in the filaments, which either directly stimulates severing or makes them more susceptible to the actin severing protein cofilin (Fig. 1B) (Mooren et al., 2009). Severing as a mechanism for actin filament reorganization stems from an observation that addition of dynamin2 and GTP to Alexa488-actin filaments formed in the presence of cortactin and Arp2/3 in vitro, led to a 'frayed' appearance of the filaments and deposition of shorter filaments on the imaging plane, as observed using TIRF microscopy (Mooren et al., 2009). Severing could also be caused via GTP hydrolysis-dependent reorganization of Factin bundles by dynamin2, increasing the susceptibility of these filaments to severing and generation of more barbed ends by cofilin, as observed in vitro from pyrene-actin polymerization assays (Mooren et al., 2009). As a result of dynamin2-dependent reorganization of lamellipodial actin networks, the F-actin may become aligned into a favorable conformation for bundling by cross-linkers. The distinct 'stripes' of lamellipodial $\alpha$-actinin fluorescence observed in kymographs of control U2-OS cells (Chapter 3, Fig. 5) could indicate a subpopulation of lamellipodial F-actin that is generated following $\alpha$-actinin bundling. 
Figure. 1: Working model for dynamin2-dependent reorganization of actin networks at the lamellipodia

(A) Dynamin2-dependent reorganization of lamellipodial actin generates a subpopulation of remodeled filaments that can be efficiently bundled by $\alpha$-actinin to create an ideal template to initiate nascent actomyosin arc formation and productive interaction with focal adhesions.

(B) Dynamin2 associates with F-actin, directly via the F-actin binding domain and indirectly via the C-terminal PRD at the distal lamellipod, along the advancing edge of a protrusion. Dynamin2 remodels and severs the actin (grey bars) in a GTP hydrolysisdependent manner. For simplicity, only dynamin2's PRD and F-actin domain are shown. 
Figure. 1: Working model for dynamin2-dependent reorganization of actin networks at the lamellipodia

A With dynamin2

Without dynamin2
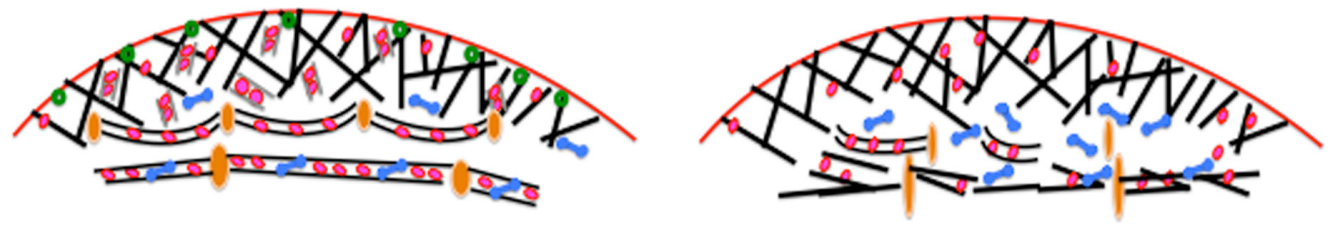

F-actin

B

Locally remodeled F-actin crosslinked with $\alpha$-actinin

- Myosin II

(1) Focal adhesion

- Dynamin2

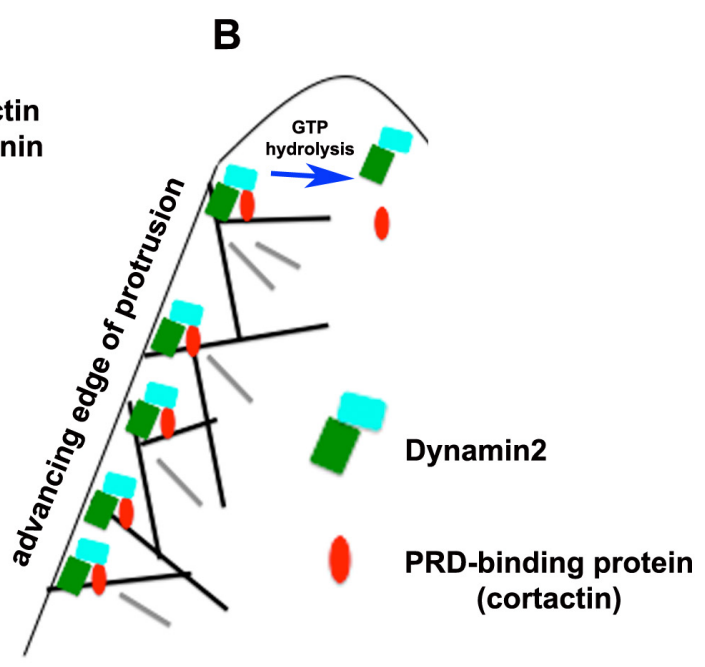




\section{GTP hydrolysis-dependent remodeling of actin networks in vivo}

My results indicate that dynamin2-dependent remodeling of lamellipodial actin is regulated by GTP hydrolysis, with neither increased nor decreased rates of GTP hydrolysis rescuing the diffuse lamellipodial $\alpha$-actinin distribution of dynamin2-depleted cells. This suggests a mechanism whereby the rate of GTP hydrolysis by dynamin2 is critical, and if the rate is higher or lower than wild-type, dynamin2 cannot efficiently remodel F-actin. Timelapse imaging suggests that a mutant with reduced hydrolysis rate, Dyn2-S61D, associates at the distal lamellipod to a greater extent than a mutant with an increased rate of hydrolysis, dyn2-T141A (Chapter 3, Fig. 10) and based on this data, I propose that rate of GTP hydrolysis influences association with lamellipodial actin networks. At a higher rate of hydrolysis, dynamin2 might dissociate from the actin network faster and therefore not remain bundled long enough to remodel the actin. On the other hand, Dyn2-S61D, due to the slower rate of hydrolysis, could remain associated with actin longer before undergoing hydrolysis-dependent reorganization and severing. The altered dynamics following reduced GTP hydrolysis rate could perturb template formation for downstream lamellar network assembly. Even though effects of Dyn2T141A and Dyn2-S61D on F-actin bundles in vitro have not yet been explored, effects of modulating the rate of GTP hydrolysis on exocytosis have been demonstrated. Rate of dynamin-dependent GTP hydrolysis, modulated using the mutants Dyn1-T141A and dyn1-T65A (Song et al., 2004), influenced the process of fusion pore expansion in chromaffin cells following exocytosis (Anantharam et al., 2011). Neither mutant behaved like the dyn1-WT. Interestingly, dynamin1 was not present at sites of exocytosis but close to those sites on the membrane (Anantharam et al., 2011), suggesting dynamin 
functioning at a distance in regulating membrane deformations during exocytosis in a GTP hydrolysis-dependent manner. One possibility is via a GTP-hydrolysis dependent remodeling of cortical actin networks to facilitate exocytosis. Therefore, a finely regulated rate of GTP hydrolysis is important and in my system, offers an interesting model of dynamin2 GTPase activity acting as a timer to determine the extent of association of dynamin2 with actin at the lamellipodia before GTP hydrolysis-dependent severing of actin.

\section{F-actin binding and $\mathrm{C}$-terminal proline rich domain}

How do direct F-actin binding and the C-terminal proline-rich domain (PRD) of dynamin2 influence actin remodeling? Dynamin2's middle domain was identified to have an F-actin binding region (Gu et al., 2010). The C-terminal PRD of dynamin2 interacts with a large repertoire of proteins that bind actin, membranes, signaling molecules like the Rac GEF vav1 and neuronal Rho GEF kalirin12, and also large scaffolding proteins that link dynamin2 to actin assembly machinery proteins like N-WASP (Qualmann et al., 2000; Razidlo et al., 2013; Schafer, 2004). Both the F-actin domain and PRD were essential for dynamin2's recruitment to the distal lamellipod. Also, a mutant lacking the PRD, dyn2- $\triangle \mathrm{PRD}$, did not restore to dynamin2-depleted cells either the diffuse lamellipodial $\alpha$-actinin distribution or the increased lamellar myosin retrograde flow

(Chapter 3, Figs. 8 and 9), suggesting that interactions mediated by dynamin2 via the PRD are critical for its function in actin reorganization.

The observations with the F-actin binding deficient mutant Dyn2- $\mathrm{K}_{5} \mathrm{E}_{5}$ (Gu et al., 2010) were intriguing. Dyn2- $\mathrm{K}_{5} \mathrm{E}_{5}$-expressing cells formed prominent $\alpha$-actinin bundles 
in the lamellipodia, rescuing the diffuse lamellipodial $\alpha$-actinin distribution in the dynamin2-depleted cells (Chapter 3, Fig. 8). However, these bundles did not engage efficiently with adhesions at the lamellipod-lamella boundary and lamellar actomyosin maintained a high rate of retrograde flow (Chapter 3, Fig. 9). Hence, the F-actin bindingdeficient mutant partially rescues the phenotypes studied, suggesting that both direct interactions of dynamin2 with F-actin and indirect interactions via PRD are required for efficient lamellipodial bundling and actomyosin assembly. It is important to note that in dyn2- $\mathrm{K}_{5} \mathrm{E}_{5}$-expressing cells, as in all dynamin2-depleted and mutant cells, the actomyosin does form eventually, but the temporal regulation is perturbed. Also, $\alpha$-actinin bundles formed in dyn2-K5E5 expressing cells are different from $\alpha$-actinin distribution in dyn2wt-expressing cells, suggesting differences in interaction of dyn2-K5e5 with actin, as compared to dyn2-WT. To understand better how Dyn2-K $\mathrm{E}_{5}$ works, further characterization is required. Dynamin2 bundles F-actin in vitro in the presence of cortactin and Arp2/3 (Mooren et al., 2009) and dynamin1 alone can bundle F-actin in vitro (Gu et al., 2010). An interesting question would be whether and how Dyn2- $\mathrm{K}_{5} \mathrm{E}_{5}$ bundles actin in vitro with cortactin and Arp2/3 and how this mutant remodels actin in the presence of GTP. I predict that the mutant would bundle F-actin because it has a Cterminal PRD through which it can interact with actin via cortactin. However, even though dyn1- $\mathrm{K}_{5} \mathrm{E}_{5}$ has wild-type rates of basal and lipid-stimulated GTP hydrolysis (Gu et al., 2010), its ability to facilitate GTP hydrolysis-dependent reorganization of F-actin is not known. Based on the observation in vivo that Dyn2- $\mathrm{K}_{5} \mathrm{E}_{5}$-expressing cells form prominent $\alpha$-actinin bundles in the lamellipodia, one prediction would be that this mutant 
remains associated with actin longer and cannot undergo GTP hydrolysis-dependent actin severing and remodeling as efficiently as dyn2 WT.

Corroboration of model with other studies

Over the past few years, several studies have implicated dynamin2 in actomyosin organization. Dynamin2 is involved in maintenance of epithelial monolayers and regulation of apical actomyosin contractility in epithelial cells (Chua et al., 2009). Dynamin2 depletion perturbed maintenance of epithelial junction architecture in MDCK cells. Dynamin2 mutants that shift the protein to a non-GTP bound state, like Dyn2K44A, induced apical constriction whereas mutants shifted to a GTP-bound state, like Dyn2-S61D, did not. Also, interactions with cortactin are required for mediating contractility in this system. Therefore, the authors postulated a concerted action for dynamin2 in regulating both actomyosin contractility and endocytosis at epithelial junctions, depending on dynamin2's nucleotide bound state. They proposed that dynamin2 could remodel F-actin via interactions with cortactin (Chua et al., 2009). It is possible that bundling and GTP hydrolysis-dependent remodeling of actin could induce localized severing of filaments that set up a template for efficient actomyosin organization and contractility in epithelial cells. This study however used dynamin2 mutants, some of them with dominant negative effects like Dyn2-K44A, and hence other effects, such as inhibited endocytosis, defects in signaling and other unaccounted effects on actin organization, cannot be ruled out.

The study by Mooren et al., demonstrating GTP hydrolysis-dependent remodeling of F-actin by dynamin2 in vitro (Mooren et al., 2009) provided key information to arrive 
at my model. They showed evidence of F-actin bundling by dynamin2, in the presence of cortactin and Arp2/3, and a possible GTP hydrolysis-dependent severing of filaments. The study also demonstrated changes in global actomyosin organization in fixed cells following depletion of dynamin2. Immunofluorescence showed a perturbed actomyosin, with enhanced association of two actomyosin proteins $\alpha$-actinin and myosin II A with the actin network in dynamin2-depleted cells, suggesting a role for dynamin2 in the global organization of the actomyosin.

Another study in podocytes showed that direct binding of dynamin to actin is critical for stress fiber organization and actin polymerization (Gu et al., 2010). Expression of dynamin1 mutants that do not bind actin led to a dramatic loss of stress fibers in dynamin2-depleted cells, whereas a mutant with enhanced actin binding could maintain stress fiber organization and also promote generation of free barbed ends, as observed from biotin G-actin labeling. The authors postulated a function for dynamin 1 in binding to actin and displacing the capping protein gelsolin to promote actin polymerization. Another interesting possibility that is not ruled out is dynamin acting as a localized actin severing protein, generating barbed ends for polymerization, which could mediate efficient stress fiber assembly. However, these effects observed in podocytes could be specific to the cell type studied.

\section{Dynamin2 in cell migration}

This research addresses a novel role for dynamin2 as a remodeler of lamellipodial and lamellar actin networks. Dynamin2 regulates assembly, organization and retrograde flow of actomyosin arcs in U2-OS cells and also influences the association of nascent 
arcs with newly forming focal adhesions. This association could slow down retrograde flow and maintain normal adhesion morphology. Dynamin2-depleted cells have high myosin retrograde flow and thinner nascent adhesions, suggesting that dynamin2 could be instrumental in setting up an actin architecture during arc formation that confers appropriate focal adhesion maturation. One important property of cells influenced by arcadhesion linkage is cell migration (Giannone et al., 2009). Appendix II of this dissertation addresses a function for dynamin2 in cell migration. My preliminary data indicate that dynamin2-depleted cells exhibit higher rate of migration during wound healing (Appendix II, Fig. 2). I therefore proposed that dynamin2-depleted cells might undergo some form of compensatory amoeboid-like migration, which some tumor cells have shown to exhibit under some conditions (Sanz-Moreno et al., 2008). Amoeboid migration is characterized by myosin contraction-dependent membrane blebbing or actin polymerization-based gliding motility and is commonly observed in dendritic cells and leukocytes (Lammermann and Sixt, 2009). Under confining conditions, which cells might experience when moving as a monolayer or through a matrix, amoeboid-like migration could predominate and the increased myosin flow and contractility could exert pressure on the membrane and push it forward. While a complete switch to a new mode of migration is unlikely, rather, partial compensation by alternate mechanisms of migration may account for the increased directed migration by dynamin2-depleted cells. One possible way of compensation is via increased rate of actin polymerization at the membrane to counter the enhanced myosin retrograde flow, as observed in dendritic cells (Renkawitz et al., 2009). One possible way to test enhanced actin assembly at 
lamellipodia is by a barbed end assay to quantitatively assess new barbed ends generated at the lamellipodia.

During random motility, dynamin2 depletion enhanced the persistence of migration, without changing the rate of migration (Appendix II, Fig. 1). Enhanced persistence could be caused by formation of protrusions predominantly on one side of the cell. Cofilin depletion exhibited increased persistence of migration in MTLn3 cells, by restricting Arp2/3 localization to the cell front and thereby limiting generation of new Arp2/3-mediated barbed ends to one side (Sidani et al., 2007). Cofilin-depleted cells cannot generate new barbed ends via severing and hence protrusions form at just one end. To test whether dynamin2 depletion affects formation of barbed ends via severing, a barbed end assay (Cai et al., 2007) can be used. Directional migration is also promoted by reducing levels of active Rac1, which decreases formation of multiple lamellipodia (Pankov et al., 2005). Even though levels of active Rac1 do not change in HT1080 cells (Appendix I, Fig. 1) as shown by pulldown experiments, more specific tests looking at active Rac1 at lamellipodial protrusions in U2-OS cells are required, using tools like a Rac1 biosensor (Machacek et al., 2009).

Finally, dynamin2 is implicated in tumor cell invasion. Dynamin2 stabilizes the Rac GEF Vav1 at the lamellipodia and promotes tumor cell invasion (Razidlo et al., 2013). Vav1 activates Rac1, which stimulates lamellipodial protrusions and invasion. Dynamin2 depletion leads to a reduction in vav1 levels (Razidlo et al., 2013). U2-OS cells however have very low levels of vav1 and these levels do not decrease following dynamin2 depletion (Chapter 3, Fig. 6). Dynamin2 can promote invasion by acting downstream of PDGFR $\alpha$-promoted tumor growth mediated by PI3 kinase and the 
phosphatase SHP2 (Feng et al., 2012). Invasion of pancreatic tumor cells in vitro and metastasis in vivo are mediated by src kinase-dependent phosphorylation of dynamin2. (Eppinga et al., 2012), with a non-phosphorylatable mutant of dynamin2 being defective in migration and invasion. An important next experiment is to understand if dynamin2 depletion in U2-OS cells influences invasion. One study demonstrated a pro-migratory function for dynamin2 during random cell motility and during directed migration into a wound (Eppinga et al., 2012), which are in contrast to my data. One explanation could be the difference in cell lines, as the other study used a highly metastatic pancreatic cancer cell line, which could have other altered properties.

Alternate mechanisms of dynamin2 influencing actin networks

In this research, I report how dynamin2 functions as an actin remodeling protein and influences lamellipodial and lamellar actin networks. However, dynamin2 is a multifaceted protein with a large number of interacting partners. Hence, other mechanisms where dynamin2 functions to modulate phosphorylation states or proteinprotein interactions cannot be ruled out.

Association of $\alpha$-actinin with actin is modulated by a variety of factors. $\alpha$-Actinin is phosphorylated by focal adhesion kinase (FAK) and phospho- $\alpha$-actinin has reduced affinity for actin (Izaguirre et al., 2001). An association of dynamin2 with FAK (Ezratty et al., 2005; Wang et al., 2011) has been shown previously and this complex could regulate $\alpha$-actinin phosphorylation. Therefore, via a dynamin2-FAK signaling pathway, dynamin 2 could limit actin cross-linking by $\alpha$-actinin at the lamellipodia and regulate filament organization. In U2-OS cells, there was no significant change in total cellular 
levels of FAK or p-FAK following dynamin2 knockdown (Chapter3, Fig. 7). Further, GFP-FAK does not localize to the distal lamellipodia in U2-OS cells, as observed for GFP-dynamin2, and hence is less likely to influence lamellipodial $\alpha$-actinin (Chapter 3, Fig. 11). However, inability to resolve the localization of FAK at the lamellipodia using a fluorescent probe is not ruled out. Phosphoinositide binding also inhibits $\alpha$-actinin-actin interaction (Fraley et al., 2003). An $\alpha$-actinin mutant that cannot interact with the phospholipid had altered association-dissociation kinetics with actin (Fraley et al., 2005). At the membrane via interactions of its $\mathrm{PH}$ domain with phospholipids, dynamin2 could modulate accessibility of phosphoinositides to $\alpha$-actinin, thereby affecting the ability of $\alpha$-actinin to bundle actin.

Dynamin2-dependent activation of the small GTPases Rac and Rho can also influence actin dynamics at the lamellipodia. Dynamin1 and dynamin2 interact with the neuronal Rho GEF kalirin12 (Xin et al., 2009) and dynamin2 interacts with and stabilizes the Rac GEF vav1 in pancreatic cancer cells (Razidlo et al., 2013). These signaling pathways regulated by dynamin 2 however could be tissue-specific.

\section{Future directions}

This research proposes a novel function for dynamin2 as a remodeler of lamellipodial actin networks that influences actomyosin assembly. Even though live cell imaging and fluorescent probes are valuable tools to understand dynamin2's action, a better understanding of mechanism can be obtained by studying remodeling of a dendritic actin network by dynamin 2 in vitro, for example by polymerizing actin comet tails attached to a polystyrene bead (Cameron et al., 2001). An interesting question would be 
to observe how dynamin2-WT bundles and remodels filaments in the dendritic network at the ultrastructural level and also how addition of the cross-linker $\alpha$-actinin influences filament organization. It will also be important to study how dyn2-T141A, dyn2-S61D, dyn2- $\Delta \mathrm{PRD}$ and dyn2- $\mathrm{K}_{5} \mathrm{E}_{5}$ influence dendritic $\mathrm{F}$-actin remodeling in vitro.

I have demonstrated a requirement for the C-terminal PRD and the F-actin binding domain for localization of dynamin2 to the distal lamellipod. However, the results with these mutants do not rule out the possibility of dynamin2 localizing to the lamellipod via a direct interaction with the advancing plasma membrane. To address this, it is important to transiently express a dynamin mutant with a pleckstrin homology $(\mathrm{PH})$ domain mutation at Lys535 - K535A - that inhibits interactions with the lipid membrane (Vallis et al., 1999), into dynamin2-depleted cells. If this mutant localizes to the distal lamellipod and also rescues standard deviation of lamellipodial $\alpha$-actinin intensity and the increased myosin retrograde flow, it will help rule out dynamin2 localization to the lamellipodia via an interaction with the plasma membrane.

Because dynamin2-depleted cells exhibit altered focal adhesion morphology and disorganized actomyosin, one hypothesis is that the adhesions do not mature efficiently due do the absence of a well-organized actomyosin (Oakes et al., 2012). To test this hypothesis, dynamin2-depleted cells can be probed for markers for mature adhesions, phospho-paxillin and phospho-focal adhesion kinase (FAK) (Oakes et al., 2012). I predict that in dynamin2-depleted cells, the newly formed adhesions closer to the cell edge at the lamellipodia-lamella interface would have reduced amounts of phosphopaxillin and phospho-FAK. Another important marker is tensin, which marks fibrillar adhesions - elaborate structures formed during ECM remodeling (Pankov et al., 2000). 
If dynamin2 is involved in efficient ECM remodeling by transducing force via a wellorganized actomyosin that can interact with adhesions, I would predict reduced tensin localization following dynamin2 depletion.

Even though this research demonstrated an endocytosis-independent function for dynamin2 in reorganization of actin networks, it is possible that dynamin2's role in endocytosis and actin remodeling are mutually non-exclusive. Dynamin is implicated in turnover of F-actin around clathrin-coated pits (Ferguson et al., 2009). Rate of GTP hydrolysis by dynamin regulates the recruitment and de-recruitment of actin to invaginating pits (Taylor et al., 2012). Therefore, dynamin2 could remodel actin networks around the invaginating vesicle to facilitate endocytosis. Another possibility is that dynamin2 could work with dynamin1 to concomitantly regulate endocytosis and actin reorganization.

\section{Concluding remarks}

This work established dynamin2 as a crucial regulator of global lamellipodial and lamellar actin networks. Dynamin2 is a key player in receptor-mediated endocytosis and in this work, I have demonstrated an endocytosis-independent function for dynamin2 in actin reorganization. Global remodeling of actin networks is relevant not only in cell migration but in other important cell biological functions like the maintenance of cell-cell junctions, endocytosis and mitosis; dynamin is implicated in all these processes (Chua et

al., 2009; Ferguson et al., 2009; Taylor et al., 2012; Thompson et al., 2002). It will be interesting to address how specific properties of dynamin2, such as rate of GTP hydrolysis and F-actin binding, modulate these important biological functions. Dynamin2 
is a multi-functional protein and through its varied roles in cell signaling, actin remodeling and endocytosis, it could regulate a large number of cell biological processes in more ways than one. 


\section{References}

Anantharam, A., Bittner, M.A., Aikman, R.L., Stuenkel, E.L., Schmid, S.L., Axelrod, D., and Holz, R.W. (2011). A new role for the dynamin GTPase in the regulation of fusion pore expansion. Mol Biol Cell 22, 1907-1918.

Anderson, T.W., Vaughan, A.N., and Cramer, L.P. (2008). Retrograde flow and myosin II activity within the leading cell edge deliver F-actin to the lamella to seed the formation of graded polarity actomyosin II filament bundles in migrating fibroblasts. Mol Biol Cell 19, 5006-5018.

Burnette, D.T., Manley, S., Sengupta, P., Sougrat, R., Davidson, M.W., Kachar, B., and Lippincott-Schwartz, J. (2011). A role for actin arcs in the leading-edge advance of migrating cells. Nat Cell Biol 13, 371-381.

Cai, L., Marshall, T.W., Uetrecht, A.C., Schafer, D.A., and Bear, J.E. (2007). Coronin 1B coordinates Arp2/3 complex and cofilin activities at the leading edge. Cell 128, 915-929.

Cameron, L.A., Svitkina, T.M., Vignjevic, D., Theriot, J.A., and Borisy, G.G. (2001). Dendritic organization of actin comet tails. Curr Biol 11, 130-135.

Chua, J., Rikhy, R., and Lippincott-Schwartz, J. (2009). Dynamin 2 orchestrates the global actomyosin cytoskeleton for epithelial maintenance and apical constriction. Proc Natl Acad Sci U S A 106, 20770-20775.

Eppinga, R.D., Krueger, E.W., Weller, S.G., Zhang, L., Cao, H., and McNiven, M.A. (2012). Increased expression of the large GTPase dynamin 2 potentiates metastatic migration and invasion of pancreatic ductal carcinoma. Oncogene 31, 1228-1241.

Ezratty, E.J., Partridge, M.A., and Gundersen, G.G. (2005). Microtubule-induced focal adhesion disassembly is mediated by dynamin and focal adhesion kinase. Nat Cell Biol 7, 581-590.

Feng, H., Liu, K.W., Guo, P., Zhang, P., Cheng, T., McNiven, M.A., Johnson, G.R., Hu, B., and Cheng, S.Y. (2012). Dynamin 2 mediates PDGFRalpha-SHP-2-promoted glioblastoma growth and invasion. Oncogene 31, 2691-2702.

Ferguson, S.M., Raimondi, A., Paradise, S., Shen, H., Mesaki, K., Ferguson, A., Destaing, O., Ko, G., Takasaki, J., Cremona, O., et al. (2009). Coordinated actions of actin and BAR proteins upstream of dynamin at endocytic clathrin-coated pits. Dev Cell $17,811-822$. 
Fraley, T.S., Pereira, C.B., Tran, T.C., Singleton, C., and Greenwood, J.A. (2005). Phosphoinositide binding regulates alpha-actinin dynamics: mechanism for modulating cytoskeletal remodeling. J Biol Chem 280, 15479-15482.

Fraley, T.S., Tran, T.C., Corgan, A.M., Nash, C.A., Hao, J., Critchley, D.R., and Greenwood, J.A. (2003). Phosphoinositide binding inhibits alpha-actinin bundling activity. J Biol Chem 278, 24039-24045.

Giannone, G., Mege, R.M., and Thoumine, O. (2009). Multi-level molecular clutches in motile cell processes. Trends Cell Biol 19, 475-486.

Gu, C., Yaddanapudi, S., Weins, A., Osborn, T., Reiser, J., Pollak, M., Hartwig, J., and Sever, S. (2010). Direct dynamin-actin interactions regulate the actin cytoskeleton. Embo J 29, 3593-3606.

Hotulainen, P., and Lappalainen, P. (2006). Stress fibers are generated by two distinct actin assembly mechanisms in motile cells. J Cell Biol 173, 383-394.

Izaguirre, G., Aguirre, L., Hu, Y.P., Lee, H.Y., Schlaepfer, D.D., Aneskievich, B.J., and Haimovich, B. (2001). The cytoskeletal/non-muscle isoform of alpha-actinin is phosphorylated on its actin-binding domain by the focal adhesion kinase. J Biol Chem $276,28676-28685$.

Lammermann, T., and Sixt, M. (2009). Mechanical modes of 'amoeboid' cell migration. Curr Opin Cell Biol 21, 636-644.

Machacek, M., Hodgson, L., Welch, C., Elliott, H., Pertz, O., Nalbant, P., Abell, A., Johnson, G.L., Hahn, K.M., and Danuser, G. (2009). Coordination of Rho GTPase activities during cell protrusion. Nature 461, 99-103.

Maciver, S.K., Wachsstock, D.H., Schwarz, W.H., and Pollard, T.D. (1991). The actin filament severing protein actophorin promotes the formation of rigid bundles of actin filaments crosslinked with alpha-actinin. J Cell Biol 115, 1621-1628.

Mooren, O.L., Kotova, T.I., Moore, A.J., and Schafer, D.A. (2009). Dynamin2 GTPase and cortactin remodel actin filaments. J Biol Chem 284, 23995-24005.

Nemethova, M., Auinger, S., and Small, J.V. (2008). Building the actin cytoskeleton: filopodia contribute to the construction of contractile bundles in the lamella. J Cell Biol $180,1233-1244$.

Oakes, P.W., Beckham, Y., Stricker, J., and Gardel, M.L. (2012). Tension is required but not sufficient for focal adhesion maturation without a stress fiber template. J Cell Biol 196, 363-374. 
Pankov, R., Cukierman, E., Katz, B.Z., Matsumoto, K., Lin, D.C., Lin, S., Hahn, C., and Yamada, K.M. (2000). Integrin dynamics and matrix assembly: tensin-dependent translocation of alpha(5)beta(1) integrins promotes early fibronectin fibrillogenesis. J Cell Biol 148, 1075-1090.

Pankov, R., Endo, Y., Even-Ram, S., Araki, M., Clark, K., Cukierman, E., Matsumoto, K., and Yamada, K.M. (2005). A Rac switch regulates random versus directionally persistent cell migration. J Cell Biol 170, 793-802.

Ponti, A., Machacek, M., Gupton, S.L., Waterman-Storer, C.M., and Danuser, G. (2004). Two distinct actin networks drive the protrusion of migrating cells. Science 305, 17821786.

Qualmann, B., Kessels, M.M., and Kelly, R.B. (2000). Molecular links between endocytosis and the actin cytoskeleton. J Cell Biol 150, F111-116.

Razidlo, G.L., Wang, Y., Chen, J., Krueger, E.W., Billadeau, D.D., and McNiven, M.A. (2013). Dynamin 2 Potentiates Invasive Migration of Pancreatic Tumor Cells through Stabilization of the Rac1 GEF Vav1. Dev Cell 24, 573-585.

Renkawitz, J., Schumann, K., Weber, M., Lammermann, T., Pflicke, H., Piel, M., Polleux, J., Spatz, J.P., and Sixt, M. (2009). Adaptive force transmission in amoeboid cell migration. Nat Cell Biol 11, 1438-1443.

Sanz-Moreno, V., Gadea, G., Ahn, J., Paterson, H., Marra, P., Pinner, S., Sahai, E., and Marshall, C.J. (2008). Rac activation and inactivation control plasticity of tumor cell movement. Cell 135, 510-523.

Schafer, D.A. (2004). Regulating actin dynamics at membranes: a focus on dynamin. Traffic 5, 463-469.

Sidani, M., Wessels, D., Mouneimne, G., Ghosh, M., Goswami, S., Sarmiento, C., Wang, W., Kuhl, S., El-Sibai, M., Backer, J.M., et al. (2007). Cofilin determines the migration behavior and turning frequency of metastatic cancer cells. J Cell Biol 179, 777-791.

Song, B.D., Leonard, M., and Schmid, S.L. (2004). Dynamin GTPase domain mutants that differentially affect GTP binding, GTP hydrolysis, and clathrin-mediated endocytosis. J Biol Chem 279, 40431-40436.

Taylor, M.J., Lampe, M., and Merrifield, C.J. (2012). A feedback loop between dynamin and actin recruitment during clathrin-mediated endocytosis. PLoS Biol 10, e1001302.

Thompson, H.M., Skop, A.R., Euteneuer, U., Meyer, B.J., and McNiven, M.A. (2002). The large GTPase dynamin associates with the spindle midzone and is required for cytokinesis. Curr Biol 12, 2111-2117. 
Tojkander, S., Gateva, G., Schevzov, G., Hotulainen, P., Naumanen, P., Martin, C., Gunning, P.W., and Lappalainen, P. (2011). A molecular pathway for myosin II recruitment to stress fibers. Curr Biol 21, 539-550.

Vallis, Y., Wigge, P., Marks, B., Evans, P.R., and McMahon, H.T. (1999). Importance of the pleckstrin homology domain of dynamin in clathrin-mediated endocytosis. Curr Biol 9, 257-260.

Vallotton, P., and Small, J.V. (2009). Shifting views on the leading role of the lamellipodium in cell migration: speckle tracking revisited. J Cell Sci 122, 1955-1958.

Wang, Y., Cao, H., Chen, J., and McNiven, M.A. (2011). A direct interaction between the large GTPase dynamin-2 and FAK regulates focal adhesion dynamics in response to active Src. Mol Biol Cell 22, 1529-1538.

Xin, X., Rabiner, C.A., Mains, R.E., and Eipper, B.A. (2009). Kalirin12 interacts with dynamin. BMC Neurosci 10,61. 


\section{Appendix I}

Dynamin2 does not influence levels of active, GTP-bound small GTPases

\section{Contributions:}

Fig. 1 B, C: Experiments, corresponding analysis and figure generation: Susanna

Blauch, Dorothy Schafer 


\section{Introduction}

The small GTPases Rac and Rho in the active, GTP-bound form are involved in regulating important functions like actin cytoskeletal organization, membrane trafficking and cell migration (Hall, 1998; Ridley, 2001). As dynamin2 is identified to interact with several proteins having Rho GEF activity (Razidlo et al., 2013; Schafer), an important control for my research is to elucidate the effect of dynamin2 depletion on levels of GTPbound Rac and Rho. Because I propose a function for dynamin2 in regulating actomyosin organization via remodeling lamellipodial actin networks, it is important to address

whether the changes observed in actin network organization following dynamin2 depletion is caused due to altered levels of active small GTPases.

GTP-bound active Rho can stimulate actomyosin contractility by turning on downstream effectors like ROCK (Rho kinase) which activates myosin II via phosphorylation of myosin light chain II (MLC2) or via inhibition of MLC phosphatase (Amano et al., 2000; Amano et al., 1996; Kaibuchi et al., 1999; Kimura et al., 1996; Ridley, 2001). Pulling force exerted by myosin II-dependent contractility can cause focal adhesion elongation (Chrzanowska-Wodnicka and Burridge, 1996). Myosin contractility can also enable pulling up the rear of the cell during cell migration (Ridley et al., 2003).

GTP-bound Rac can promote actin polymerization via several potential mechanisms. GTP-Rac can activate Arp2/3 and promote dendritic actin polymerization at the leading edge of the lamellipodia. Active Rac can produce PtdIns(4,5)P2 which in turn removes capping protein from barbed ends of actin filaments. GTP-Rac also activates LIMK, which phosphorylates and inactivates cofilin (Ridley, 2001). However, Rac1 could inhibit cell migration by formation of several lamellipodial protrusions all around 
the cell and hence function as an internal regulator determining the switch between random and directed cell migration (Pankov et al., 2005).

\section{Results and Discussion}

Dynamin2 does not influence levels of active small GTPases

Chapter 3 details a function for dynamin2 in actomyosin organization and contractility, via remodeling lamellipodial actin networks. Because the small GTPases Rac and Rho influence actin polymerization and myosin contractility (Ridley, 2001), an important control experiment is to address whether depletion of dynamin2 alters the cellular levels of GTP-bound Rac and Rho. In general, active Rac is involved with formation of protrusions via initiation of actin polymerization. Using Rac pulldown assay, I showed that dynamin2 depletion did not change either total or active Rac1 levels (Fig. 1A), as quantified from whole cell lysates in the fibrosarcoma cell line HT1080, indicating that dynamin2 does not influence activation of Rac1. Even though my research was performed in U2-OS cells, I chose HT1080 for the Rac pulldown assay because I could not obtain clear bands corresponding to Rac1 using western blotting in U2-OS cells, while HT1080 express high levels of Rac1 (Niggli et al., 2009). However, to address whether localized levels of active Rac change at lamellipodial protrusions in U2OS cells, more sensitive tools will need to be used. One example is a GTP-Rac biosensor (Machacek et al., 2009), which will help determine if levels of active Rac are altered at lamellipodial protrusions following dynamin2 depletion. A recent study demonstrated a function for dynamin2 in stabilizing the Rac GEF vav1 and promoting invasion of pancreatic cancer cells via Rac activation (Razidlo et al., 2013). The authors 
demonstrated that dynamin2 depletion led to reduction in total vav1 levels. In U2-OS cells, as shown in chapter 3, vav1 levels were low. Furthermore, vav1 levels did not decrease following dynamin2 depletion, potentially ruling out a function for dynamin2 in promoting Rac activation via stabilization of its GEF vav1 in U2-OS cells, which was the model postulated by the authors for panc-1 cells (Razidlo et al., 2013).

GTP-bound, active Rho promotes actomyosin contraction and enables cell migration by promoting focal adhesion maturation and providing force for pulling the rear of the cell (Raftopoulou and Hall, 2004). Because dynamin2 regulates actomyosin contractility and focal adhesion morphology, it is important to determine whether these effects are mediated via modulation of active Rho. As a first step, chapter 3 showed that dynamin2 depletion did not alter levels of phosphorylated, active downstream targets of Rho, like phospho-myosin light chain (p-MLC) and phospho-cofilin. However, to directly observe GTP-Rho in U2-OS cells, a previously characterized Raichu RhoA FRET-based biosensor (Heasman et al., 2010; Makrogianneli et al., 2009) was used and FRET efficiency measured using Fluorescence Lifetime Imaging microscopy (FLIM) in U2-OS cells (Fig. 1 B, C). Dynamin2 depletion did not significantly change the FRET efficiency (Fig. 1C), while FRET efficiency in control cells transfected with a dominant negative biosensor (RhoDN) was significantly lower than the wild-type biosensor (Fig. 1C). This analysis however measured FRET efficiency over the entire cell. More intricate analysis, looking specifically at levels of GTP-Rho at lamellipodial protrusions (Machacek et al., 2009) in control and dynamin2-depleted cells will better address a function for dynamin 2 in modulating Rho activity. 
Figure. 1: Dynamin2 depletion does not alter levels of active GTPases.

(A) Levels of active, GTP-bound Rac do not change following dynamin2 depletion in HT1080 cells, as shown by the ratio of Active Rac/Total Rac. The protein levels in the gels were quantified using the 'analyze gels' tool in ImageJ.

(B) Representative control and dynamin2-depleted U2-OS cells showing the lifetimes of donor fluorescence.

(C) FRET efficiency of the entire cell calculated from donor lifetime (refer Materials and Methods) and compared between control, dynamin2-depleted and control cells expressing a dominant negative Rho biosensor that does not undergo FRET due to lack of interaction between the domains.

(Experiments in $\mathrm{B}$ and $\mathrm{C}$ performed by Susanna Blauch and Dorothy Schafer) 
Figure. 1: Dynamin2 depletion does not alter levels of active GTPases.

A

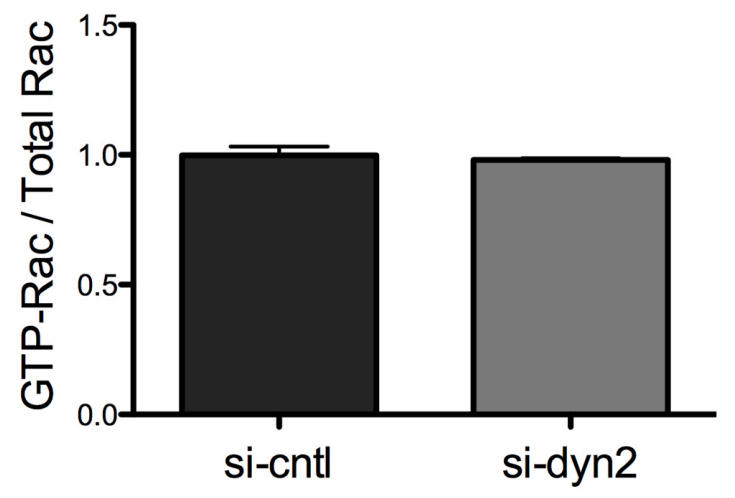

B

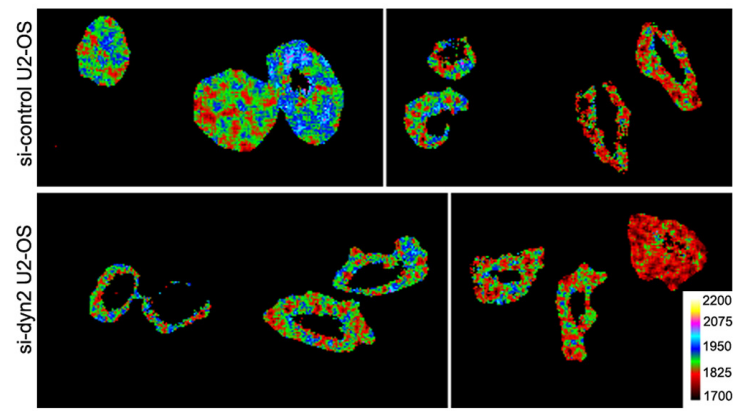

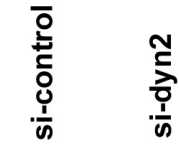

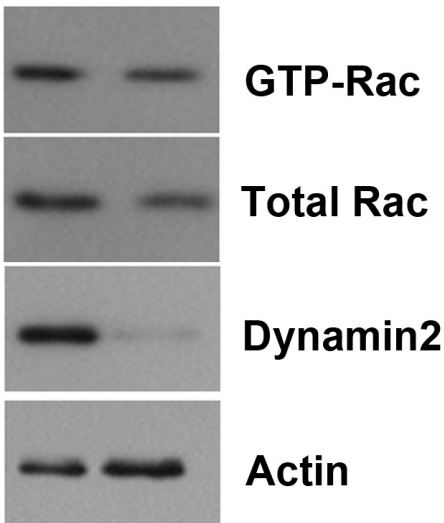

C

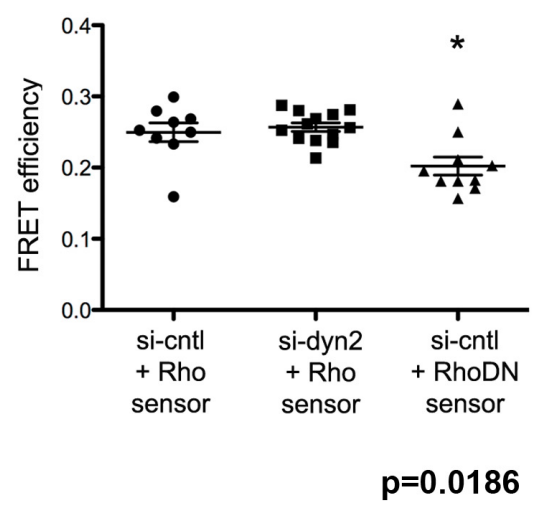




\section{Conclusion}

These data show that dynamin2 depletion does not change global cellular levels of GTP-Rac in HT1080 cells and GTP-Rho in U2-OS cells. However, further experimentation is required to specifically address whether levels of active GTPases change locally at lamellipodial protrusions. These preliminary results indicate that dynamin2 regulates lamellipodial actin organization and protrusive behavior independent of Rac activation, and influences actomyosin organization and contractility via a mechanism independent of activation of Rho-GTP.

\section{Materials and Methods}

Cell culture and transfections

U2-OS and HT1080 cells were plated in DMEM and or MEM media respectively, supplemented with $10 \%$ fetal bovine serum, glutamine, sodium pyruvate and nonessential amino acids. siRNAs $(2 \mu \mathrm{g})$ were delivered into U2-OS cells using an Amaxa Nucleofector II, program X-001, and nucleofection kit V (Amaxa Biosystems, Lonza), and into HT1080 cells using program L-005 and nucleofection solution T, according to the manufacturer's protocol. One million cells were used per nucleofection. Cells were used in experiments $48 \mathrm{~h}$ after nucleofection.

Multiphoton fluorescence lifetime imaging microscopy using the Rho biosensor in U2$\underline{\text { OS cells }}$

To probe RhoA activation, a Raichu RhoA FRET-based biosensor, modified with GFP and mRFP as described (Heasman et al., 2010; Makrogianneli et al., 2009), together with 
negative control RhoA-T19N and baseline probe GFP, was used. Two-photon (2p) timecorrelated single photon counting (TCSPC) FLIM was used to quantify RhoA biosensor FRET, the setup of which is described in detail in (Chen and Periasamy, 2004; Sun et al., 2011). Briefly, the FLIM system was implemented on a Biorad Radiance 2100 confocal/multiphoton microscope (Nikon TE300 inverted) system. A Coherent 10W Verdi pumped tunable (700-1000 nm) pulsed laser tuned to $870 \mathrm{~nm}$ was used. All FLIM images were acquired using a Nikon 60X/1.2 NA water-immersion objective lens. The accumulation time of each FLIM image was 120 seconds to provide enough photon counts for accurate fluorescence decay fitting. FLIM images were analyzed using $\mathrm{BH}$ SPCImage software. The FRET efficiency was estimated based on "1-(TauDA/TauD)", where TauDA is the lifetime of the donor (GFP) in presence of the acceptor (i.e., the GFP-mRFP-RhoA biosensor); TauD is the donor-alone lifetime ( $2464 \mathrm{ps})$, measured from the cells expressing GFP only. Fluorescence lifetime Images obtained from the SPCImage software were exported and processed in ImageJ for display.

\section{Quantitation of active Rac1 in HT1080 cells}

To quantify active Rac1, we modified the protocol from (Benard and Bokoch, 2002; del Pozo et al., 2000; Pellegrin and Mellor, 2008). $1 \times 10^{6}$ HT1080 cells $/ 10 \mathrm{~cm}$ dish were washed twice with ice-cold PBS and lysed with $1 \mathrm{ml}$ ice-cold lysis buffer (50mM Tris $\mathrm{pH} 7.5,10 \mathrm{mM}$ MgCL2, 200mM NaCl, 1\% NP-40, 5\% glycerol, 1mM PMSF, $1 \mu \mathrm{g} / \mathrm{ml}$ leupeptin and aprotinin) containing $20 \mu \mathrm{g}$ GST-PBD (Pak binding domain). After centrifugation and a whole-lysate sample collected, $800 \mu$ of the clarified lysate was added to glutathione-Sepharose beads previously washed in ice-cold lysis buffer. The 
suspension was mixed by rotation for 45 minutes at $4^{\circ} \mathrm{C}$. The beads were washed thrice in wash buffer (25mM Tris, $\mathrm{pH} 7.6,30 \mathrm{mM} \mathrm{MgCl} 2,40 \mathrm{mM} \mathrm{NaCl}, 1 \% \mathrm{NP}-40,1 \mathrm{mM}$ DTT, $1 \mu \mathrm{g} / \mathrm{ml}$ leupeptin and aprotinin) followed by two washes in wash buffer without NP-40. 2X SDS buffer was added to the beads and the sample was heated at $95^{\circ} \mathrm{C}$ for 5 minutes, centrifuged at $14,000 \mathrm{rpm}$ for 2 minutes and the supernatant stored at $-20^{\circ} \mathrm{C}$ until analysis for Rac1 (clone 23A8, Millipore) using western blots. 


\section{References}

Amano, M., Fukata, Y., and Kaibuchi, K. (2000). Regulation and functions of Rhoassociated kinase. Exp Cell Res 261, 44-51.

Amano, M., Ito, M., Kimura, K., Fukata, Y., Chihara, K., Nakano, T., Matsuura, Y., and Kaibuchi, K. (1996). Phosphorylation and activation of myosin by Rho-associated kinase (Rho-kinase). J Biol Chem 271, 20246-20249.

Benard, V., and Bokoch, G.M. (2002). Assay of Cdc42, Rac, and Rho GTPase activation by affinity methods. Methods Enzymol 345, 349-359.

Chen, Y., and Periasamy, A. (2004). Characterization of two-photon excitation fluorescence lifetime imaging microscopy for protein localization. Microsc Res Tech 63, 72-80.

Chrzanowska-Wodnicka, M., and Burridge, K. (1996). Rho-stimulated contractility drives the formation of stress fibers and focal adhesions. J Cell Biol 133, 1403-1415.

del Pozo, M.A., Price, L.S., Alderson, N.B., Ren, X.D., and Schwartz, M.A. (2000). Adhesion to the extracellular matrix regulates the coupling of the small GTPase Rac to its effector PAK. EMBO J 19, 2008-2014.

Hall, A. (1998). Rho GTPases and the actin cytoskeleton. Science 279, 509-514.

Heasman, S.J., Carlin, L.M., Cox, S., Ng, T., and Ridley, A.J. (2010). Coordinated RhoA signaling at the leading edge and uropod is required for $\mathrm{T}$ cell transendothelial migration. J Cell Biol 190, 553-563.

Kaibuchi, K., Kuroda, S., and Amano, M. (1999). Regulation of the cytoskeleton and cell adhesion by the Rho family GTPases in mammalian cells. Annu Rev Biochem 68, 459486.

Kimura, K., Ito, M., Amano, M., Chihara, K., Fukata, Y., Nakafuku, M., Yamamori, B., Feng, J., Nakano, T., Okawa, K., et al. (1996). Regulation of myosin phosphatase by Rho and Rho-associated kinase (Rho-kinase). Science 273, 245-248.

Machacek, M., Hodgson, L., Welch, C., Elliott, H., Pertz, O., Nalbant, P., Abell, A., Johnson, G.L., Hahn, K.M., and Danuser, G. (2009). Coordination of Rho GTPase activities during cell protrusion. Nature 461, 99-103.

Makrogianneli, K., Carlin, L.M., Keppler, M.D., Matthews, D.R., Ofo, E., Coolen, A., Ameer-Beg, S.M., Barber, P.R., Vojnovic, B., and Ng, T. (2009). Integrating receptor signal inputs that influence small Rho GTPase activation dynamics at the immunological synapse. Mol Cell Biol 29, 2997-3006. 
Niggli, V., Schlicht, D., and Affentranger, S. (2009). Specific roles of Rac1 and Rac2 in motile functions of HT1080 fibrosarcoma cells. Biochem Biophys Res Commun 386, 688-692.

Pankov, R., Endo, Y., Even-Ram, S., Araki, M., Clark, K., Cukierman, E., Matsumoto, K., and Yamada, K.M. (2005). A Rac switch regulates random versus directionally persistent cell migration. J Cell Biol 170, 793-802.

Pellegrin, S., and Mellor, H. (2008). Rho GTPase activation assays. Curr Protoc Cell Biol Chapter 14, Unit 1418.

Raftopoulou, M., and Hall, A. (2004). Cell migration: Rho GTPases lead the way. Dev Biol 265, 23-32.

Razidlo, G.L., Wang, Y., Chen, J., Krueger, E.W., Billadeau, D.D., and McNiven, M.A. (2013). Dynamin 2 Potentiates Invasive Migration of Pancreatic Tumor Cells through Stabilization of the Rac1 GEF Vav1. Dev Cell 24, 573-585.

Ridley, A.J. (2001). Rho GTPases and cell migration. J Cell Sci 114, 2713-2722.

Ridley, A.J., Schwartz, M.A., Burridge, K., Firtel, R.A., Ginsberg, M.H., Borisy, G., Parsons, J.T., and Horwitz, A.R. (2003). Cell migration: integrating signals from front to back. Science 302, 1704-1709.

Schafer, D.A. (2004). Regulating actin dynamics at membranes: a focus on dynamin. Traffic 5, 463-469.

Sun, Y., Day, R.N., and Periasamy, A. (2011). Investigating protein-protein interactions in living cells using fluorescence lifetime imaging microscopy. Nat Protoc 6, 1324-1340. 


\section{Appendix II}

\section{Regulation of cell migration by dynamin2}

\section{Contributions:}

Fig. 2: Experiment, corresponding analysis and figure generation: Eugene Harris, Dorothy Schafer 


\section{Introduction}

Cell migration is a complex process, with several steps and regulated by a large number of effectors. It involves protrusion of the cell front, anchoring this protrusion to the underlying substratum through focal adhesions and retraction of the rear (Ridley et al., 2003). Several factors can determine how a cell migrates - the nature of the substrate on which it migrates, surrounding cells and signaling via Rho GTPases (Friedl and Wolf, 2010; Pankov et al., 2005; Raftopoulou and Hall, 2004). Another crucial component of cell migration is protrusive behavior of the cell at the leading edge. Because dynamin2 localizes to the advancing edges of lamellipodial protrusions, in this section I will focus on how dynamin2 regulates cell migration, possibly via modulating protrusive behavior.

A protrusion occurs via fast actin polymerization at the lamellipodial edge and is coupled to depolymerization at the rear of the lamellipodia. This process called 'actin treadmilling' regenerates actin monomers for further actin polymerization at the leading cell edge (Le Clainche and Carlier, 2008). At the lamellipodia, the actin nucleating protein Arp2/3 maintains a dendritic, branched actin network, stabilized by proteins like cortactin (Weaver et al., 2001). A protrusion is stabilized by formation of focal complexes that provide an anchorage to the substratum, and coupled with force provided by actomyosin contractility, enables the cell body to translocate.

A role for dynamin in cell migration could be attributed to its ability to influence turnover of focal adhesions \{(Ezratty et al., 2005), stabilization of the Rac GEF vav1, which promotes actin polymerization, cell migration and invasion (Razidlo et al., 2013) 
or by regulating actin organization at the lamellipodia through interactions via dynamin's PRD (Kruchten and McNiven, 2006).

\section{Results and Discussion}

Dynamin2 regulates random cell migration and directed migration into a wound

Chapter 3 details a function for dynamin2 in regulating global actomyosin assembly, organization and contractility and coupling of the actomyosin with focal adhesions. Because actomyosin contractility can influence cell migration (Gupton and Waterman-Storer, 2006), I investigated how dynamin2 regulated random cell migration using a random cell motility assay, and directed migration using a wound-healing assay.

Depleting dynamin2 altered neither the total distance traveled over four hours nor the rate of migration during random migration (Fig. 1A). However, many dyn2-depleted cells maintained a more directed path during migration (Fig. 1B). To quantify directed migration, the net distance and persistence were measured. The net distance, which is the shortest distance between the first and last time points (Fig. 1C), was significantly higher following dynamin2 depletion in a population size of 8-10 cells (Fig. 1A). As a result, dynamin2-depleted cells also had a significantly higher persistence (Net distance / total distance) than control cells. In a wound-healing assay, dynamin2-depleted cells closed the wound at a slightly faster rate than control cells (Fig. 2). Together, these results suggest a role for dynamin2 in regulating directed cell migration.

In chapter 3, I have shown that dynamin2 is important for efficient actomyosin assembly and organization. How could dynamin2 influence cell migration via regulation of the actomyosin? The poorly organized actomyosin network in dynamin2-depleted cells 
may not link efficiently with focal adhesions, thereby maintaining a high retrograde flow and low adhesion strength. Weak adhesion strength can initiate an alternate amoeboidlike migration (Lammermann and Sixt, 2009). Traction force microscopy can be used to measure traction stress exerted on a compliant substrate by focal adhesions at the cell front during migration (Gardel et al., 2008). Amoeboid migration is characterized by a blebbing-based motility or by an actin polymerization-based gliding motility. Dendritic cells maintain their rate of migration even when myosin retrograde flow is increased, by enhancing rate of actin polymerization (Renkawitz et al., 2009). U2-OS cells could undergo a similar adaptation to maintain cell migration when dynamin2 is depleted.

A compensatory amoeboid migration has been observed in tumor cell lines like HT1080 migrating in a three dimensional environment, when proteolysis is blocked (Wolf et al., 2003). Since tumor cells undergo amoeboid migration during invasion through a three-dimensional matrix (Wolf et al., 2003), an interesting experiment would be to study the behavior and migration rate of U2-OS cells depleted of dynamin2, migrating in a 3D environment through a matrigel-coated chamber. One outcome could be that dynamin2-depleted U2-OS cells exhibit enhanced invasion rate, due to their ability to adapt to an amoeboid-like migration and invade matrigel better. The opposite outcome - reduced invasion following dynamin 2 depletion - is also possible and has been demonstrated in metastatic cancer cell lines (Eppinga et al., 2012; Razidlo et al., 2013). In metastatic cancer cells, dynamin 2 expression increases migration and invasion (Eppinga et al., 2012; Razidlo et al., 2013). If dynamin2-depleted U2-OS cells exhibit reduced invasion rate, it could also be due to an inability to effectively degrade the matrix and efficiently remodel the actomyosin during invasion. 
Increased persistence of migration during random motility could be caused due to formation of protrusions predominantly on one side of the cell as opposed to multiple lamellipodial protrusions all around the cell. This phenotype of increased persistence has been observed in cofilin-depleted cells (Sidani et al., 2007) where cofilin regulates actin severing and localization of Arp2/3 and generates actin branches for nucleation. Following cofilin depletion, Arp2/3 localizes to the front of the cell, promoting actin nucleation just at the cell front and hence protrusions in one direction. Localization of Arp2/3 could be compared between control and dynamin2-depleted cells. Further experiments, like a barbed end assay, could determine whether barbed ends for further actin polymerization were generated preferentially at one end in dynamin2-depleted cells. It is important to emphasize here that the analysis of random cell motility has been performed on a population of 8-10 cells each under control and dynamin2-depleted conditions. A larger dataset will have to be analyzed to more conclusively determine dynamin2's effect on random cell migration. 
Figure. 1: Dynamin2 regulates persistence of migration during random cell motility.

(A) Total distance, net distance, persistence and speed of migration of individual cells. Each data point on the graph depicts a single cell. A total of 8-10 cells analyzed per set. $* \mathrm{p}<0.05$.

(B) Depiction of paths followed by representative control and dynamin2-depleted cells during random migration over four hours.

(C) Cartoon depicting the parameters used for analysis of migratory behavior in U2OS cells. 
Figure. 1: Dynamin2 regulates persistence of migration during random cell motility.
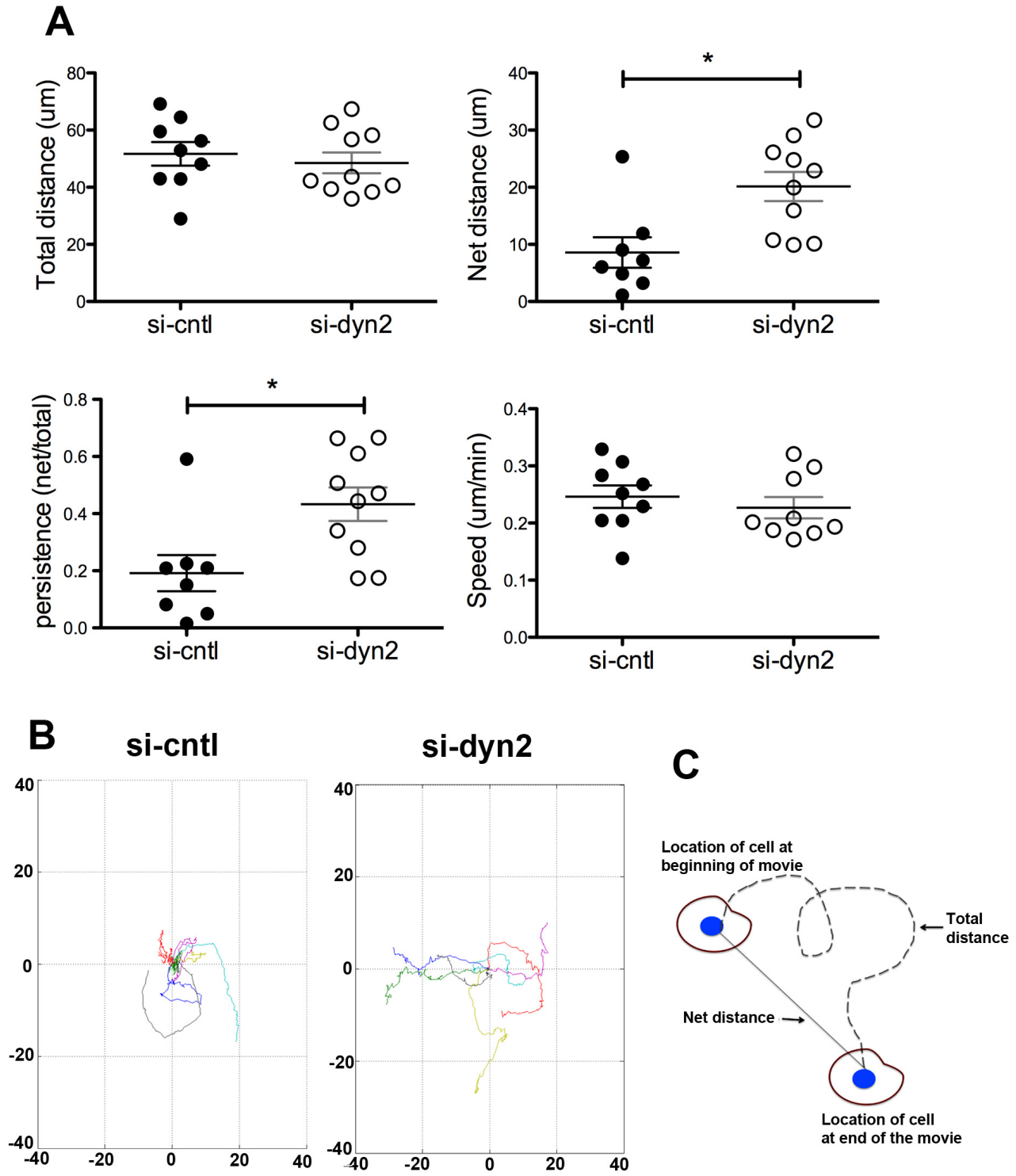

Paths followed during random cell motility 
Figure. 2: Dynamin2 influences directed cell migration into a wound.

Closure of scratch wound induced in a confluent monolayer of control and dynamin2depleted U2-OS cells, imaged over 20 hours. Data is obtained from four independent experiments (Experiments and analysis performed by Eugene Harris, Dorothy Schafer) 
Figure. 2: Dynamin2 influences directed cell migration into a wound.

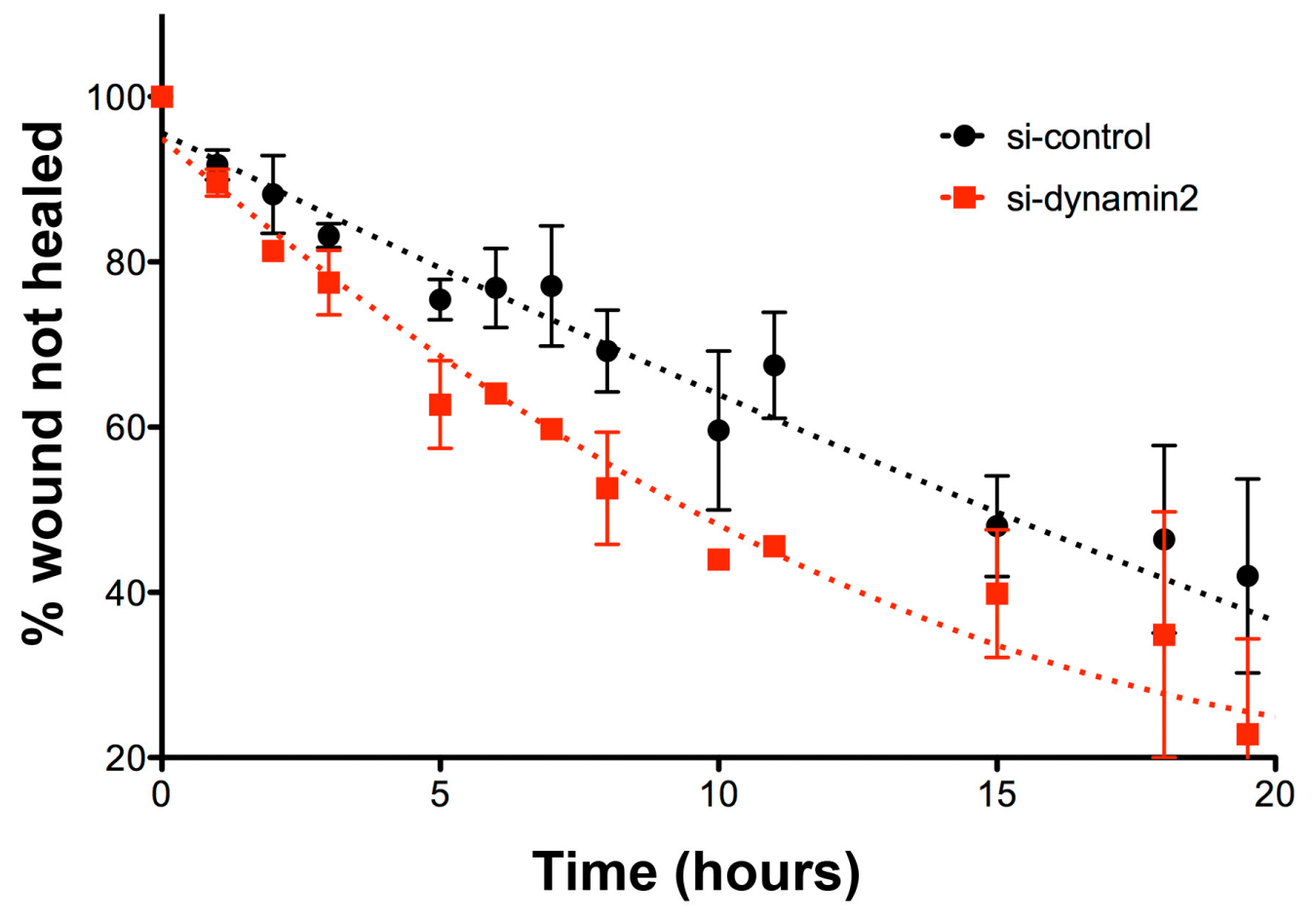


Dynamin2 regulates protrusive behavior in randomly migrating cells

Protrusive behavior at the lamellipodia is an important component of cell migration. Because dynamin2 localizes to the advancing edges of protrusions, I asked how protrusive behavior is modified following dynamin2 depletion. I analyzed distance and duration of lamellipodial protrusions in control, dynamin2-depleted and dynamin2depleted cells rescued with mCh-dyn2-WT. Dynamin2-depleted cells formed shorter, less persistent protrusions (Fig. 3A, B), which could be rescued with mCh-dyn2-WTexpressing cells (Fig. 3A). Therefore, dynamin2 could influence formation of stable protrusions, potentially by functioning as a scaffold for polymerizing actin by localizing along advancing edges of protrusions. Another interpretation could be that the increased retrograde flow in dynamin2-depleted cells negatively regulated forward advancement of the membrane. Because dynamin2-depleted cells still migrate, albeit more persistently, it suggests that protrusive behavior may not adversely affect cell migration. Instead, stable protrusion formation mediated by dynamin 2 could be involved in maintaining the random motility exhibited by control U2-OS cells. 
Figure. 3: Dynamin2 regulates lamellipodial protrusive behavior.

(A) Distance and duration of protrusions measured in control ( $\mathrm{n}=5$ cells), dynamin2depleted ( $\mathrm{n}=6$ cells) and dynamin2-depleted cells rescued with mCh-dyn2-WT $(\mathrm{n}=5$ cells $) . * * \mathrm{p}<0.005$. All the cells also express GFP- $\alpha$-actinin.

(B) Representative kymographs from control and dynamin2-depleted cells showing how the various parameters were measured. $y$ is the protrusion distance, $x$ the duration of protrusions and $y / x$ is the rate of protrusion. Scale bar: $1 \mu \mathrm{m}$. 
Figure. 3: Dynamin2 regulates lamellipodial protrusive behavior.

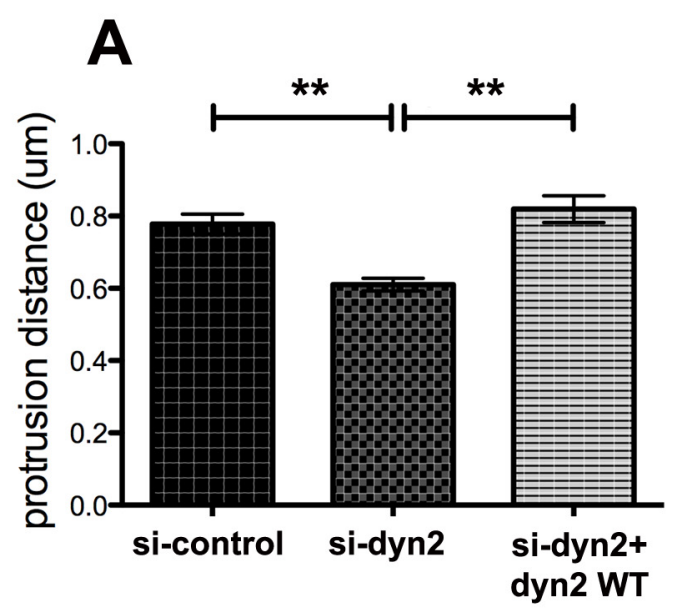

B
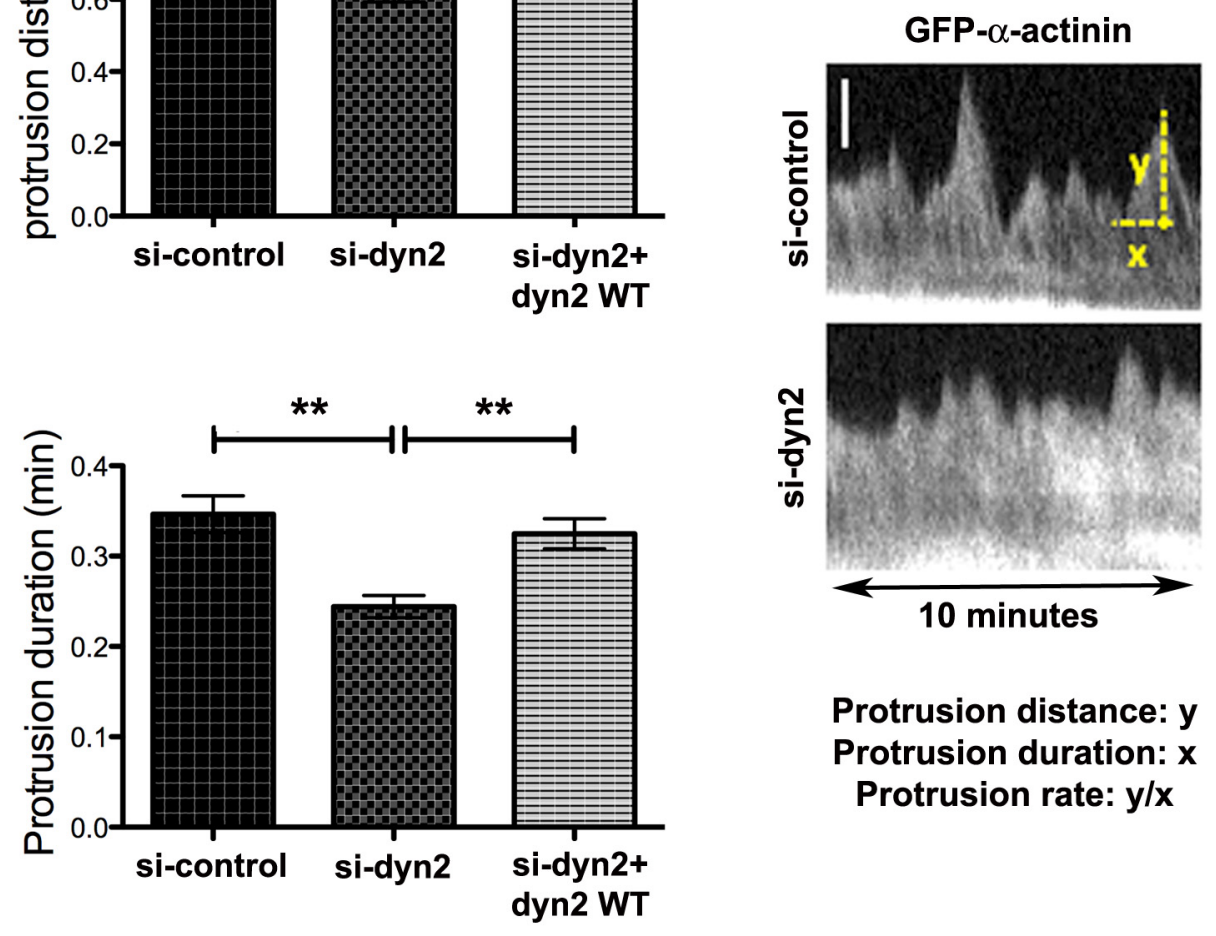

Protrusion distance: y Protrusion duration: $x$ Protrusion rate: $y / x$ 


\section{Conclusion}

In Appendix II, I report a function for dynamin2 in regulating both random and directed cell migration. Dynamin2-depleted cells exhibit enhanced persistence of migration during random motility and increased rate of cell migration into a wound. The increase in directionality of migration could be caused due to formation of lamellipodial protrusions preferentially at one end of the cell instead of all around the cell, thereby making cell migration more directed. Enhanced directed migration has been observed following depletion of the actin severing protein cofilin (Sidani et al., 2007), due to persistent formation of protrusions on one side of the cell. It is possible that dynamin2, through its role as an actin bundling and severing protein, could influence generation of new barbed ends and hence new actin polymerization at protrusions. Another scenario that can enhance directed migration is modulation of levels of GTP-bound active Rac. Decreased levels of active Rac reduce formation of peripheral lamellipodia in fibroblasts and instead form lamellipodia in one direction, increasing directed migration (Pankov et al., 2005). Dynamin2 depletion however does not change overall levels of active Rac in HT1080 cells, as shown in Appendix I.

An interesting possibility for the slightly enhanced rate of cell migration into a wound exhibited by dynamin2-depleted cells could be that the cells develop some form of compensatory pathway so that cell migration is not reduced due to perturbed actomyosin organization and contractility. Dendritic cells maintain the same rate of cell migration even when the myosin retrograde flow is increased, by enhancing rate of actin polymerization at the lamellipodia (Renkawitz et al., 2009). Dynamin2-depleted U2-OS cells could similarly modulate actin polymerization at protruding lamellipodia to 
maintain similar rates of cell migration as control cells. Also, when dynamin2-depleted cells migrate in confinement, which could occur during directed migration as a confluent monolayer, the cells could push against the confinement, in this case, neighboring cells, and maintain cell migration even when myosin retrograde flow and interaction of adhesions with the overlying actomyosin network are perturbed (Hawkins et al., 2009).

In conclusion, dynamin2's role in regulating actomyosin organization and contractility via remodeling lamellipodial actin networks could potentially maintain random cell motility by influencing lamellipodial protrusive behavior. Following dynamin2 depletion, cells could develop a compensatory pathway so that cell migration is not adversely perturbed in the presence of enhanced actomyosin contractility and a weak adhesion-actomyosin network linkage.

\section{Experimental methods}

\section{Cell culture and transfections}

U2-OS cells were plated in DMEM media, supplemented with $10 \%$ fetal bovine serum, glutamine, sodium pyruvate and non-essential amino acids. siRNAs $(2 \mu \mathrm{g})$ were delivered into U2-OS cells using an Amaxa Nucleofector II, program X-001, and nucleofection kit V (Amaxa Biosystems, Lonza), according to the manufacturer's protocol. One million cells were used per nucleofection. Cells were used in experiments $48 \mathrm{~h}$ after nucleofection. 1-1.2 $\mathrm{g}$ of plasmid were transfected into cells using FuGene transfection reagent, according to the manufacturer's protocol.

\section{Microscopy}


Glass-bottomed $35 \mathrm{~mm}$ culture dishes (MatTek Corporation) or glass coverslips were coated with $2 \mu \mathrm{g} / \mathrm{ml}$ fibronectin (Sigma) overnight at $4^{\circ} \mathrm{C}$. U2-OS cells were plated in supplemented DMEM media for 3-4 hours before imaging. 30 minutes before imaging, DMEM media was replaced with 'movie media' (phenol-red free MEM supplemented with $10 \%$ fetal bovine serum, glutamine and 20mM HEPES). Confocal microscopy was performed on a Zeiss confocal microscope with a Nakagawa spinning disc, equipped with a $63 \mathrm{X}$ oil objective lens (for confocal) or $25 \mathrm{X}$ oil objective lens (for DIC), lasers to excite GFP (488nm) and mCherry (587nm), an Orca R2 camera (Hamamatsu) and controlled using software from Metamorph. A definite focus device (Carl Zeiss) was used to maintain focus during single focal plane imaging. The following frame rates were used: random cell motility assay in U2-OS cells: $2 \mathrm{~min} /$ frame for 4 hours; cell edge dynamics analysis in U2-OS cells: $5 \mathrm{sec} /$ frame for 10 minutes.

\section{$\underline{\text { Random cell motility assay in U2-OS cells }}$}

U2-OS cells plated at $7 \times 10^{4}$ cells/ glass-bottomed $35 \mathrm{~mm}$ culture dishes (MatTek Corporation) were imaged at $25 \mathrm{X}$ using Differential Interference Contrast (DIC) microscopy, using an Orca R2 camera (Hamamatsu). ImageJ analysis tools were used for measurement of the various cell motility parameters. The $\mathrm{X}$ and $\mathrm{Y}$ coordinates (in $\mu \mathrm{m}$ ) of the centroid of the cell were obtained at each time point. To quantify the total distance traveled, the sum of the distances between each pair of adjacent time points was obtained. For net distance traveled, the absolute difference in distance between the first and last time points was obtained. Persistence is the Net distance/total distance. 


\section{Wound healing assay in U2-OS cells}

A wound was scratched with a $200 \mu$ pipette tip on a confluent monolayer of control and dynamin2-depleted U2-OS cells. The wound was imaged immediately after scratching and at different time points over 20 hours. At least 3 different regions of the wound were imaged at each time point. Data were plotted as a percentage of wound closed over time.

\section{Analysis of cell edge dynamics in U2-OS cells}

Control and dynamin2-depleted U2-OS cells were co-transfected with GFP- $\alpha$ actinin and $\mathrm{mCh}-\mathrm{C} 1$ vector, and for rescue, dynamin2-depleted cells were co-transfected with GFP- $\alpha$-actinin along with mCh-dyn2 WT. For analysis of protrusion and retraction dynamics, ImageJ was used to plot kymographs from pixel-wide wide lines drawn perpendicular to the cell edge along regions exhibiting protrusive activity. Lines were drawn along protrusions and retractions in the kymographs to acquire length and angle measurements and a custom program run to obtain the rate, duration and distance of protrusions as described (Bear et al., 2002; Cai et al., 2007; Hinz et al., 1999). 


\section{References}

Bear, J.E., Svitkina, T.M., Krause, M., Schafer, D.A., Loureiro, J.J., Strasser, G.A., Maly, I.V., Chaga, O.Y., Cooper, J.A., Borisy, G.G., et al. (2002). Antagonism between Ena/VASP proteins and actin filament capping regulates fibroblast motility. Cell 109, 509-521.

Cai, L., Marshall, T.W., Uetrecht, A.C., Schafer, D.A., and Bear, J.E. (2007). Coronin 1B coordinates Arp2/3 complex and cofilin activities at the leading edge. Cell 128, 915-929.

Eppinga, R.D., Krueger, E.W., Weller, S.G., Zhang, L., Cao, H., and McNiven, M.A. (2012). Increased expression of the large GTPase dynamin 2 potentiates metastatic migration and invasion of pancreatic ductal carcinoma. Oncogene 31, 1228-1241.

Ezratty, E.J., Partridge, M.A., and Gundersen, G.G. (2005). Microtubule-induced focal adhesion disassembly is mediated by dynamin and focal adhesion kinase. Nat Cell Biol 7, 581-590.

Friedl, P., and Wolf, K. (2010). Plasticity of cell migration: a multiscale tuning model. J Cell Biol 188, 11-19.

Gardel, M.L., Sabass, B., Ji, L., Danuser, G., Schwarz, U.S., and Waterman, C.M. (2008). Traction stress in focal adhesions correlates biphasically with actin retrograde flow speed. J Cell Biol 183, 999-1005.

Gupton, S.L., and Waterman-Storer, C.M. (2006). Spatiotemporal feedback between actomyosin and focal-adhesion systems optimizes rapid cell migration. Cell 125, 13611374.

Hawkins, R.J., Piel, M., Faure-Andre, G., Lennon-Dumenil, A.M., Joanny, J.F., Prost, J., and Voituriez, R. (2009). Pushing off the walls: a mechanism of cell motility in confinement. Phys Rev Lett 102, 058103.

Hinz, B., Alt, W., Johnen, C., Herzog, V., and Kaiser, H.W. (1999). Quantifying lamella dynamics of cultured cells by SACED, a new computer- assisted motion analysis. Exp Cell Res 251, 234-243.

Kruchten, A.E., and McNiven, M.A. (2006). Dynamin as a mover and pincher during cell migration and invasion. J Cell Sci 119, 1683-1690.

Lammermann, T., and Sixt, M. (2009). Mechanical modes of 'amoeboid' cell migration. Curr Opin Cell Biol 21, 636-644.

Le Clainche, C., and Carlier, M.F. (2008). Regulation of actin assembly associated with protrusion and adhesion in cell migration. Physiol Rev 88, 489-513. 
Pankov, R., Endo, Y., Even-Ram, S., Araki, M., Clark, K., Cukierman, E., Matsumoto, K., and Yamada, K.M. (2005). A Rac switch regulates random versus directionally persistent cell migration. J Cell Biol 170, 793-802.

Raftopoulou, M., and Hall, A. (2004). Cell migration: Rho GTPases lead the way. Dev Biol 265, 23-32.

Razidlo, G.L., Wang, Y., Chen, J., Krueger, E.W., Billadeau, D.D., and McNiven, M.A. (2013). Dynamin 2 Potentiates Invasive Migration of Pancreatic Tumor Cells through Stabilization of the Rac1 GEF Vav1. Dev Cell 24, 573-585.

Renkawitz, J., Schumann, K., Weber, M., Lammermann, T., Pflicke, H., Piel, M., Polleux, J., Spatz, J.P., and Sixt, M. (2009). Adaptive force transmission in amoeboid cell migration. Nat Cell Biol 11, 1438-1443.

Ridley, A.J., Schwartz, M.A., Burridge, K., Firtel, R.A., Ginsberg, M.H., Borisy, G., Parsons, J.T., and Horwitz, A.R. (2003). Cell migration: integrating signals from front to back. Science 302, 1704-1709.

Sidani, M., Wessels, D., Mouneimne, G., Ghosh, M., Goswami, S., Sarmiento, C., Wang, W., Kuhl, S., El-Sibai, M., Backer, J.M., et al. (2007). Cofilin determines the migration behavior and turning frequency of metastatic cancer cells. J Cell Biol 179, 777-791.

Weaver, A.M., Karginov, A.V., Kinley, A.W., Weed, S.A., Li, Y., Parsons, J.T., and Cooper, J.A. (2001). Cortactin promotes and stabilizes Arp2/3-induced actin filament network formation. Curr Biol 11,370-374.

Wolf, K., Mazo, I., Leung, H., Engelke, K., von Andrian, U.H., Deryugina, E.I., Strongin, A.Y., Brocker, E.B., and Friedl, P. (2003). Compensation mechanism in tumor cell migration: mesenchymal-amoeboid transition after blocking of pericellular proteolysis. $\mathrm{J}$ Cell Biol 160, 267-277. 